\title{
Will Pitch Velocity Remain Consistent Throughout a Competitive Baseball Season Following Blood Flow Restriction: an Observational Study
}

\author{
Mackenzie Marie Evans \\ mme0016@mix.wvu.edu
}

Follow this and additional works at: https://researchrepository.wvu.edu/etd

Part of the Medical Physiology Commons, Other Medical Sciences Commons, and the Sports Sciences Commons

\section{Recommended Citation}

Evans, Mackenzie Marie, "Will Pitch Velocity Remain Consistent Throughout a Competitive Baseball Season Following Blood Flow Restriction: an Observational Study" (2020). Graduate Theses,

Dissertations, and Problem Reports. 7522.

https://researchrepository.wvu.edu/etd/7522

This Thesis is protected by copyright and/or related rights. It has been brought to you by the The Research Repository @ WVU with permission from the rights-holder(s). You are free to use this Thesis in any way that is permitted by the copyright and related rights legislation that applies to your use. For other uses you must obtain permission from the rights-holder(s) directly, unless additional rights are indicated by a Creative Commons license in the record and/ or on the work itself. This Thesis has been accepted for inclusion in WVU Graduate Theses, Dissertations, and Problem Reports collection by an authorized administrator of The Research Repository @ WVU. For more information, please contact researchrepository@mail.wvu.edu. 
Will Pitch Velocity Remain Consistent Throughout a Competitive Baseball Season Following Blood Flow Restriction: an Observational Study

Mackenzie Evans, ATC

Thesis submitted to the

College of Physical Activity and Sports Sciences

At West Virginia University

In partial fulfillment of the requirements

For the degree of

Master of Science

in

Athletic Training

Michelle A. Sandrey, PhD, ATC, Chair

William G. Hornsby, PhD

Jackson C. Stembridge, MS

Department of Sport Sciences

Morgantown, West Virginia

2020

Keywords: blood flow restriction, baseball, pitcher, fastball, velocity, recovery, fatigue

Copyright 2020 Mackenzie Evans 


\section{ABSTRACT \\ Will Pitch Velocity Remain Consistent Throughout a Competitive Baseball Season Following Blood Flow Restriction: an Observational Study}

\section{Mackenzie Evans, ATC}

Context: Blood flow restriction (BFR) has previously been used in a rehabilitation setting to decrease the external load applied to post-surgical patients and those with chronic debilitating conditions. BFR in a performance context has been explored minimally, particularly as it applies to baseball pitching over time. Objective: The purpose of this study was to retrospectively investigate the trend in throwing velocity of fastballs with BFR implementation over one competitive season. Design: This study was a retrospective longitudinal design on pitching velocity of fastballs throughout a competitive season. Setting: The data collection and analysis was completed at a mid-Atlantic university. Patients or other participants: Four Division I starting baseball pitchers. Inclusion criteria were a starting baseball pitcher during the 2018-2019 season, on the official baseball roster for that season and had a sport physical on file. Exclusion criteria were any individual not meeting the inclusion criteria. Intervention: Data analysis was performed using data recorded by Trackman during home games throughout the 2018-2019 competitive baseball season. The fastball pitch velocity data was deidentified and participants were assigned a subject number. BFR was applied by the full-time baseball athletic trainer to all 4 pitchers following pitching bouts during all 60 games during the 2018-2019 baseball competitive season. The BFR occlusion protocol varied based on the limb size/arm girth of the individual, with a goal of reaching approximately 50 percent of the limb occlusive pressure. The cuff was then be inflated for five minutes with no concomitant exercise and then deflated for one minute. This process of inflation followed by deflation was repeated three times, for a total treatment duration of 18 minutes, in which, the cuff was inflated for 15 minutes. Main Outcome Measures: Pitching velocity of fastballs, number of fastballs thrown and number of innings pitched were all recorded and analyzed for each subject. Results: The highest fastball pitch velocity average was $96.0 \pm 0.9 \mathrm{mph}$, which occurred during game 11 in inning 1 and inning 3 $(96.0 \pm 0.7 \mathrm{mph})$, pitched by subject 1 . The lowest fastball pitch velocity average was $86.4 \pm 1.2$ mph, which occurred during game 4 , inning 2 pitched by subject 2 . In all games except for games 2, 6, 7, 10 and 14, the fastball pitching velocity over the game remained consistent with a slight decrease. In all games except for games 2, 6 and 15, the fastball pitching velocity averages by inning over the game remained relatively consistent with a decrease. Games 7 and 10 depicted the most consistent fastball pitches thrown over the course of a game, with no detectable increase or decrease from the line of best fit. Subject 1 pitched in four games and had the highest overall pitch velocity average throughout the 4 games with $92.45 \pm 0.95 \mathrm{mph}$ and was also the most consistent fastball pitcher on average. Subject 3 exhibited the second highest overall pitch velocity over a span of 5 games, with an average pitch velocity of $92.06 \pm 1.2 \mathrm{mph}$. Next was subject 4 , with an average pitch velocity of $88.86 \pm 1.0 \mathrm{mph}$. Causality could not be determined whether the consistency in fastball velocity was related to the use of a BFR recovery treatment due to deviations from the BFR protocol outlined in the intervention. Conclusions: The number of fastballs thrown decreased throughout a single game progression, but the average number of fastballs thrown throughout the season remained consistent from game to game. The average pitching velocity of fastballs thrown remained consistent throughout individual games. 
Furthermore, average fastball pitching velocity remained consistent with a slight increase in throwing velocity among individual subjects. 


\section{ACKNOWLEDGEMENTS}

I would like to thank my mom, dad and sister for supporting me throughout my educational endeavors throughout my entire life. Nightly phone calls with you are what got me through my graduate school experiences, and I would not be where I am today without your constant encouragement and unconditional love. I know I can be difficult to placate at times, but your persistence, patience and kindness is invaluable. I appreciate all you do for me, both emotionally and literally, even if I do not always show my appreciation.

I would like to thank Allison Hetrick for giving me guidance throughout the past two years I spent at West Virginia University. You inspired me to pursue research on blood flow restriction and continue to provide me feedback and help me with problem-solving in other facets of graduate life. Thank you for always playing devil's advocate in order to give me a greater perspective on everything. You always have creative ideas in research and otherwise, and I could not have asked for a wiser supervisor during my graduate experience.

I would like to thank Dr. Sandrey, Dr. Hornsby and Cole Stembridge for being a part of my thesis committee. I would like to further thank Dr. Sandrey for being my committee chair and program director who pushed me to succeed throughout my time in the MSAT program. I know this semester did not turn out the way anyone had intended, but I appreciate your patience and understanding. You were all outstanding resources and made great contributions to shape my thesis into a work I am very proud of.

I would also like to thank Jack Watson and other staff/faculty of CPASS for welcoming me into their CPASS family. The hallways of CPASS would have been very lonely without your constant check-ins and advice. All of my encounters were positive and regardless of how many interactions we had, I will cherish my memories with you all wherever I end up next.

Lastly, thank you to Yum Nguyen. Thank you for making time to meet with me during your busy schedule to help me with anything I was stressing about. You taught me so much about people, research and the complexities of the knee and I only wish you got here sooner so I could have learned more. I envy the students who get to be a part of your entry-level program because they are certainly in for a treat getting to learn from you. 


\section{TABLE OF CONTENTS}

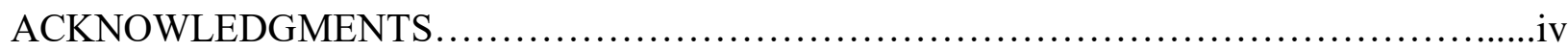

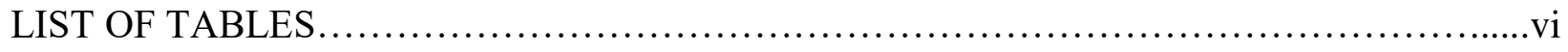

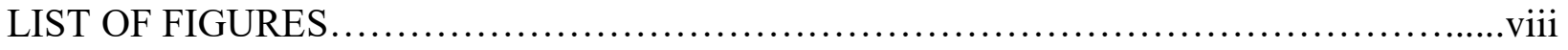

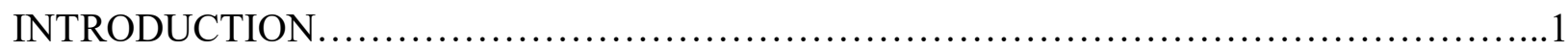

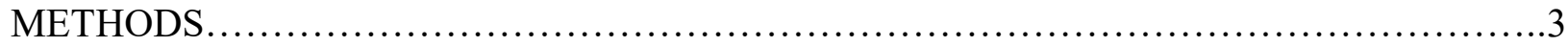

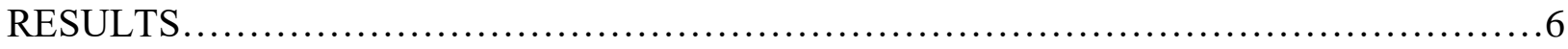

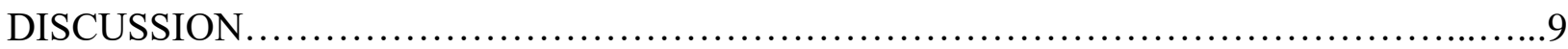

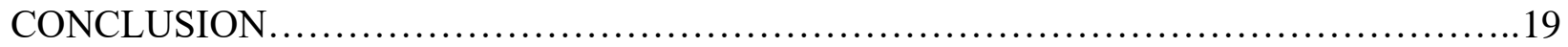

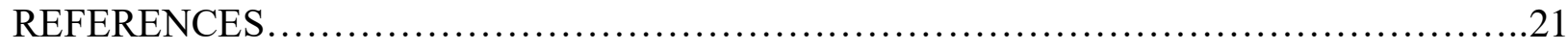

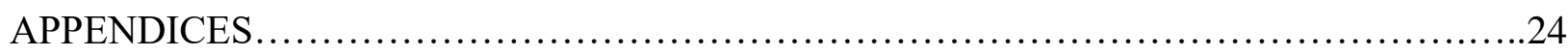

APPENDIX A THE PROBLEM.....................................................

APPENDIX B LITERATURE REVIEW ..........................................

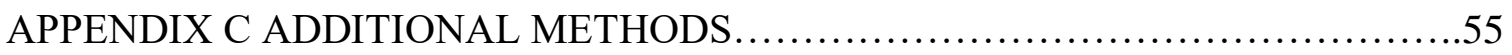

APPENDIX D ADDITIONAL RESULTS ......................................56

APPENDIX E RECOMMENDATIONS FOR FUTURE RESEARCH..................91

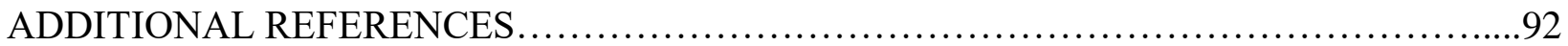




\section{LIST OF TABLES}

Table B1. Studies on BFR Based on Lower Extremity Exercise Programs........................37

Table B2. Studies on BFR Based on Upper Extremity Exercise Programs......................41

Table C1. Sample Data Collection Sheet .............................................. 55

Table D1. Fastball Pitch Count, Velocity Chart, Descriptive Statistics...........................56

Table D2. Fastball Pitch Count, Velocity Chart, Descriptive Statistics..........................56

Table D3. Fastball Pitch Count, Velocity Chart, Descriptive Statistics..........................57

Table D4. Fastball Pitch Count, Velocity Chart, Descriptive Statistics Continued................57

Table D5. Fastball Pitch Count, Velocity Chart, Descriptive Statistics..........................58

Table D6. Fastball Pitch Count, Velocity Chart, Descriptive Statistics..........................58

Table D7. Fastball Pitch Count, Velocity Chart, Descriptive Statistics Continued.................59

Table D8. Fastball Pitch Count, Velocity Chart, Descriptive Statistics...........................60

Table D9. Fastball Pitch Count, Velocity Chart, Descriptive Statistics..........................61

Table D10. Fastball Pitch Count, Velocity Chart, Descriptive Statistics Continued...............61

Table D11. Fastball Pitch Count, Velocity Chart, Descriptive Statistics..........................61

Table D12. Fastball Pitch Count, Velocity Chart, Descriptive Statistics Continued..............62

Table D13. Fastball Pitch Count, Velocity Chart, Descriptive Statistics.........................63

Table D14. Fastball Pitch Count, Velocity Chart, Descriptive Statistics Continued..............63

Table D15. Fastball Pitch Count, Velocity Chart, Descriptive Statistics.........................64

Table D16. Fastball Pitch Count, Velocity Chart, Descriptive Statistics Continued...............64

Table D17. Fastball Pitch Count, Velocity Chart, Descriptive Statistics........................65

Table D18. Fastball Pitch Count, Velocity Chart, Descriptive Statistics Continued...............66

Table D19. Fastball Pitch Count, Velocity Chart, Descriptive Statistics............................66

Table D20. Fastball Pitch Count, Velocity Chart, Descriptive Statistics Continued..............67

Table D21. Fastball Pitch Count, Velocity Chart, Descriptive Statistics......................... 67

Table D22. Fastball Pitch Count, Velocity Chart, Descriptive Statistics Continued...............68

Table D23. Fastball Pitch Count, Velocity Chart, Descriptive Statistics......................... 68

Table D24. Fastball Pitch Count, Velocity Chart, Descriptive Statistics Continued..............69 
Table D25. Fastball Pitch Count, Velocity Chart, Descriptive Statistics......................69

Table D26. Fastball Pitch Count, Velocity Chart, Descriptive Statistics Continued.............70

Table D27. Fastball Pitch Count, Velocity Chart, Descriptive Statistics..................... 70

Table D28. Fastball Pitch Count, Velocity Chart, Descriptive Statistics Continued..............71

Table D29. Fastball Pitch Count, Velocity Chart, Descriptive Statistics Continued.............72 


\section{LIST OF FIGURES}

Figure B1. Fatigue, Performance and Injury Rates Linked............................48

Figure D1. Game 1, Subject 4, Overall Fastball Pitch Velocity (mph).......................72

Figure D2. Game 1 Averages, Subject 4, Fastball Pitch Velocity Averages by Inning (mph)...73

Figure D3. Game 2, Subject 4, Overall Fastball Pitch Velocity (mph).......................73

Figure D4. Game 2 Averages, Subject 4, Fastball Pitch Velocity Averages by Inning (mph)...73

Figure D5. Game 3, Subject 1, Overall Fastball Pitch Velocity (mph).......................74

Figure D6. Game 3 Averages, Subject 1, Fastball Pitch Velocity Averages by Inning (mph)....74

Figure D7. Game 4, Subject 2, Overall Fastball Pitch Velocity (mph) ......................75

Figure D8. Game 4 Averages, Subject 2, Fastball Pitch Velocity Averages by Inning (mph)....75

Figure D9. Game 5, Subject 3, Overall Fastball Pitch Velocity (mph)......................76

Figure D10. Game 5 Averages, Subject 3, Fastball Pitch Velocity Averages by Inning (mph)...77

Figure D11. Game 6, Subject 4, Overall Fastball Pitch Velocity (mph)......................77

Figure D12. Game 6 Averages, Subject 4, Fastball Pitch Velocity Averages by Inning (mph)...77

Figure D13. Game 7, Subject 1, Overall Fastball Pitch Velocity (mph).....................78

Figure D14. Game 7 Averages, Subject 1, Fastball Pitch Velocity Averages by Inning (mph)...78

Figure D15. Game 8, Subject 2, Overall Fastball Pitch Velocity (mph)......................79

Figure D16. Game 8 Averages, Subject 2, Fastball Pitch Velocity Averages by Inning (mph)...79

Figure D17. Game 9, Subject 3, Overall Fastball Pitch Velocity (mph)......................79

Figure D18. Game 9 Averages, Subject 3, Fastball Pitch Velocity Averages by Inning (mph)...80

Figure D19. Game 10, Subject 4, Overall Fastball Pitch Velocity $(\mathrm{mph}) \ldots \ldots \ldots \ldots \ldots \ldots \ldots . . . . . . . . .80$

Figure D20. Game 10 Averages, Subject 4, Fastball Pitch Velocity Averages by Inning (mph)..80

Figure D21. Game 11, Subject 1, Overall Fastball Pitch Velocity (mph)....................81

Figure D22. Game 11 Averages, Subject 1, Fastball Pitch Velocity Averages by Inning (mph)..81

Figure D23. Game 12, Subject 2, Overall Fastball Pitch Velocity (mph)....................82

Figure D24. Game 12 Averages, Subject 2, Fastball Pitch Velocity Averages by Inning (mph)..82

Figure D25. Game 13, Subject 3, Overall Fastball Pitch Velocity (mph)....................82

Figure D26. Game 13 Averages, Subject 3, Fastball Pitch Velocity Averages by Inning (mph)..83 
Figure D27. Game 14, Subject 1, Overall Fastball Pitch Velocity (mph)....................83

Figure D28. Game 14 Averages, Subject 1, Fastball Pitch Velocity Averages by Inning (mph)..83

Figure D29. Game 15, Subject 2, Overall Fastball Pitch Velocity (mph)....................84

Figure D30. Game 15, Subject 4, Overall Fastball Pitch Velocity $(\mathrm{mph}) \ldots \ldots \ldots \ldots \ldots \ldots . . . \ldots . . . .84$

Figure D31. Game 15 Averages, Subject 2, Fastball Pitch Velocity Averages by Inning (mph)..85

Figure D32. Game 15 Averages, Subject 4, Fastball Pitch Velocity Averages by Inning (mph)..85

Figure D33. Game 16, Subject 3, Overall Fastball Pitch Velocity (mph)....................85

Figure D34. Game 16 Averages, Subject 3, Fastball Pitch Velocity Averages by Inning (mph)..86

Figure D35. Game 17, Subject 3, Overall Fastball Pitch Velocity (mph).....................86

Figure D36. Game 17 Averages, Subject 3, Fastball Pitch Velocity Averages by Inning (mph)..86

Figure D37. Subject 1 Fastball Pitch Velocity Averages by Game (mph)...................87

Figure D38. Subject 1 Fastball Pitch Velocity Averages by Inning (mph)...................87

Figure D39. Subject 2 Fastball Pitch Velocity Averages by Game (mph)....................87

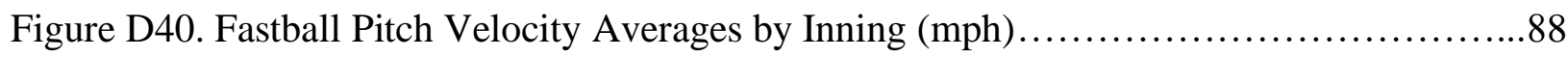

Figure D41. Subject 3 Fastball Pitch Velocity Averages by Game $(\mathrm{mph}) \ldots \ldots \ldots \ldots \ldots \ldots \ldots . \ldots 8$

Figure D42. Subject 3 Fastball Pitch Velocity Averages by Inning (mph)...................89

Figure D43. Subject 4 Fastball Pitch Velocity Averages by Game (mph).....................89

Figure D44. Subject 4 Averages by Inning......................................... 90 


\section{INTRODUCTION}

Blood flow restriction (BFR) is a tourniquet device-assisted partial occlusion of blood flow to/from an extremity. ${ }^{1}$ The BFR cuff can be applied for any of the limbs, either the arms or the legs in order to promote physiological changes in the extremity that leads to strength and/or hypertrophic gains. There is great variability in the cuffs available, in terms of brand and cuff width. Examples of the cuffs include a pneumatic cuff, ${ }^{2,3}$ tourniquet ${ }^{2,3}$ or wrap sometimes known as a KAATSU device, which is manufactured by that company. ${ }^{1}$

BFR in its primitive form was known as KAATSU training and was performed at a low intensity. KAATSU training was invented in Japan and used among bodybuilders as a health and legal alternative to supplement heavy lifts, and still observe an increase in muscle mass to enhance performance. ${ }^{1}$ Currently, BFR has been used in the rehabilitation setting. Those that can benefit from BFR in rehabilitation include patients in the military population, ${ }^{4}$ patients with chronic pain conditions such as osteoarthritis, ${ }^{1,5}$ sarcopenia, ${ }^{5}$ rheumatoid arthritis, ${ }^{5}$ patellofemoral pain, ${ }^{6}$ and patients undergoing post-operative rehabilitation (including anterior cruciate ligament rupture, ${ }^{3,7}$ or Achilles tendon rupture ${ }^{4}$ ). BFR is considered useful in patients with some type of preexisting condition in that BFR is used as an alternative to traditional highresistance or high-load training associated with rehabilitation. ${ }^{1,2,4}$ For these patients, traditional high-resistance training is not indicated for any number of reasons. For post-operative patients, there are healing concerns involved with tissues vulnerable to high-impact forces. High-impact forces may negatively affect the structure operated on, if high-resistance exercise is implemented prematurely. ${ }^{1,2}$ In those patients with the other debilitating conditions, restricted activity level is likely due to pain. ${ }^{6,8}$ Unfortunately, for patients with chronic pain conditions, strengthening via resistance training is necessary to reduce disability, but traditional resistance training produces 
intolerable pain leading to decreased patient compliance. ${ }^{8}$ BFR exercise is introduced at a lowload, which places fewer stresses on injured or painful tissues. While low-load training without BFR typically does not produce significant changes in strength, low-load training with BFR produces results similar to high-resistance training. ${ }^{2,3}$

On the other spectrum, BFR not only produces muscle adaptations comparable to traditional high resistance training, but also simulates a muscle environment with decreased oxygen available due to blood occlusion. Low-load BFR training is beneficial as a supplement to sport-specific training and decreases injury risk as compared to traditional resistance training because the external load is minmized. ${ }^{9}$ The observed increase in strength and muscle mass can translate into sport-specific, functional activities such as 5 meter sprint, 20 meter shuttle run and 505 agility.$^{10} 505$ agility is a test in which an individual sprints 5 meters, with an infrared timing gate placed at the halfway point, cuts and then returns to the starting point. ${ }^{10}$ More research needs to be conducted on the upper extremity, but a study by Manimmanakorn et al. ${ }^{10}$ demonstrated that bench press with BFR can increase chest girth greater than without BFR.

As can be seen, BFR has previously been used in a strengthening and a rehabilitative context. However, the concepts and implications of BFR can also be applied to a performance context, although research is limited in this area.${ }^{11}$ BFR could be useful, particularly in baseball performance because of the unique demands placed on baseball pitchers. Division I collegiate baseball teams play an average of over 50 games per season. ${ }^{12}$ Pitchers throw a variable number and types of pitches each inning that can lead to fatigue. Pitching is a high-intensity motion that leads to fatigue by increasing the lactic acid in blood circulation of the involved extremities (particularly the upper extremity) ${ }^{13}$ Fatigue is caused by a decrease in blood flow and oxygen, which diminishes the body's ability to replace glycogen stores for energy ${ }^{13}$ Furthermore, fatigue 
can contribute to decreased motor ability, decreased acuity in the joints of the upper extremity, poor mechanics and, in turn, injury. ${ }^{13,14,15}$

Goals of performance enhancement training include not only strength and hypertrophy, but also in safe training and injury prevention. One way to prevent injury is to combat fatigue that inevitably occurs from the volume of pitches thrown. ${ }^{13,16}$ Due to the prevalence of injury and the unique nature of pitching, BFR could be useful in supplementing athletic practices and lifting sessions to enhance performance. BFR could simulate a fatigued extremity, like that experienced during a game, so that athletes could train in a fatigued state. With a strict protocol as well as close monitoring, BFR can be implemented safely and effectively. Since the demand placed on baseball pitchers is unique to other sports, the performance enhancement training should also be unique. ${ }^{13}$ More research is needed to determine the long-term effects of BFR throughout a baseball season to observe any increase in pitching performance. Thus, to fully observe the trend in performance with BFR implementation, longitudinal studies must be conducted throughout a full baseball season or multiple baseball seasons. Therefore, the purpose of this study was to retrospectively investigate the trend in throwing velocity of fastballs over a competitive baseball season, with the starting pitchers undergoing BFR treatment.

\section{METHODS}

\section{Design}

This study used a retrospective investigative data collection approach to observe any change in pitching velocity when BFR was implemented. Variables recorded included subject number and average pitching velocity of fastballs by pitcher, inning and game. Variables evaluated for the four pitchers were average pitching velocity of fastballs and number of pitches thrown by inning, each game and games over the 2018-2019 season. 


\section{Participants}

There were four participants between the ages of 18-22 that were starting baseball pitchers at one mid-Atlantic university. Exact ages as well as height and weight were removed during the de-identification of the data. Inclusion criteria were a starting baseball pitcher during the 2018-2019 season, on the official baseball roster for that season, had a sport physical on file and pitched at least 21 fastballs in four or more games. The exclusion criterion was any individual not meeting the inclusion criteria. This study was approved by the Institutions Office of Research Integrity and Compliance.

\section{Instruments}

TrackMan: A statistics and analytics software (TrackMan, Vedbæk, Denmark) that incorporates military-grade 3D Doppler radar. TrackMan tracks both pitching and hitting data based off the Cartesian location at home plate and involves a large radar panel installed behind home plate in Monongalia County Ballpark. TrackMan tracks pitch velocity, spin rate, tilt, extension, effective velocity, zone velocity, release height, release side, vertical break, horizontal break, exit speed, launch angle, direction, spin rate, hang time, bearing, and distance. ${ }^{17}$ As compared to manual baseball statistics recording, the average error for pitching and hitting speed is 2.3 percent and 2.8 percent, respectively. ${ }^{18}$

Procedures

Data for pitch velocity was collected for all home games during the 2018-2019 competitive baseball season using TrackMan software, with the exception of 5 games, where data was either not applicable to the four subjects (they did not pitch in those games) or data was corrupted. TrackMan data has been collected and analyzed by the team manager at one midAtlantic university baseball team since the 2017 baseball season. Due to confidentiality concerns, 
the athletic trainer for the baseball team obtained the analyzed TrackMan data. TrackMan pitch velocity output data for the four individuals meeting the inclusion criteria were provided after output was deidentified. The four participants were denoted as "subject 1", "subject 2", and so on. Data per inning, game and season collected from TrackMan were entered in Table C1. This included number of innings pitched, number of fastballs thrown (organized by inning), velocity of fastballs and average velocity of fastballs (organized by inning and game).

The athletic trainer with the baseball team has a research background and training in BFR. Implementation of BFR protocol started during the beginning of the 2018-2019 competitive season. Following one or multiple pitching bouts, the pitcher would exit the game and not return for the remainder of the game. At this point, the head athletic trainer applied a BFR SmartCuff on the proximal portion of the pitching arm and inflated it to a certain pressure, with the intention of occluding blood flow. The athletic trainer inflated it to a minimum of $90 \mathrm{mmHg}$ and a maximum of $140 \mathrm{mmHg}$, and based the pressure on limb size, with a goal of reaching approximately 50 percent of limb occlusive pressure. Smaller limbs (in terms of circumference and muscle tone) were subject to a lower pressure, and the opposite for larger arms. The cuff was then inflated for five minutes with no concomitant exercise and then deflated for one minute. This process of inflation followed by deflation was repeated three times, for a total treatment duration of 18 minutes, in which, the cuff was inflated for 15 minutes. Deviations in this procedure were due to multiple pitchers leaving the game within a short timeframe (less than 18 minutes apart). Additionally, there was only one SmartCuff device, so the athletic trainer was limited in supplies and could only apply it to one pitcher at a time. If a player left the game due to injury, BFR was not applied.

Statistical Analysis 
Since this study was not meant to establish causality, the trends in pitching velocity were observed over the baseball season using descriptive statistics including averages, minimums, maximums, ranges and standard deviations. Average velocity was calculated for each inning to demonstrate the trend throughout each home game of the season. Average velocity was also calculated for each game and presented as each subject's inning and game played. A line graph was used to note average velocity for each home game. The data points were plotted on a line graph to determine whether pitch velocity, increased, decreased or remained consistent. A line of best fit was also generated to assist in evaluation of graphs. Microsoft Excel 2010 (Microsoft, Bellevue, Washington) was used for all analyses.

\section{RESULTS}

Tables D1 through D29 depict all of the fastball pitch counts and velocities as well as descriptive statistics including maximum, minimum, range, average and standard deviation for each inning. Figures D1 through D36 were organized by game number, while figures D37 through D44 were organized by subject number.

\section{Fastball Pitch Count and Velocity}

The game with the lowest minimum was a fastball pitched by subject 4 in game 10 with a velocity of $81.1 \mathrm{mph}$ (Table D16). The game with the highest maximum mph was a fastball pitched by subject 1 in game 11 with a velocity of $97.8 \mathrm{mph}$ (Table D17). Range is defined as the maximum data point minus the minimum data point. The widest range in fastball pitch velocity was $11.5 \mathrm{mph}$ which occurred during game 5 , inning 2 pitched by subject 2 (Table D6). The smallest range in fastball pitch velocity was $0.6 \mathrm{mph}$ which occurred during game 4 , inning 3 pitched by subject 3 (Table D5). The highest average was $96.0 \pm 0.9 \mathrm{mph}$, which occurred during 
game 11 in inning 1 and inning 3 (96.0 \pm 0.7 ), pitched by subject 1 (Table D17). The lowest average was $85.8 \pm 0.0 \mathrm{mph}$ which occurred during game 4 , inning 3 , pitched by subject 2 (Table D5). Since there was only one pitch thrown that inning by subject 1 , this does not represent a true average. A pitch thrown at $86.4 \pm 1.2 \mathrm{mph}$ would be the true lowest average. This occurred during game 4, inning 2 pitched by subject 2 (Table D5).

Average Fastball Pitching Velocity by Game and Inning

The fastball pitching velocity observed by game and inning was relatively consistent as depicted by the linear lines of best fit for Figures D1 through D36. Any increases or decreases mentioned were very subtle, particularly among pitches thrown throughout an entire game. Since there are more data points on the figures demonstrating entire games (nine innings), the changes in fastball pitching velocity were not as drastic as those that were present when very few innings were pitched. In games 1, 3-5, 8, 9, 11-13 and 15-17, the fastball pitching velocities over the game remained consistent, with a very slight decrease (Figures D1, D5, D7, D9, D15, D17, D21, D23, D25, D29, D31, D33, respectively). In games 1-5, 7-13 and 15-17 the fastball pitching velocity averages over the game remained consistent with a decrease (Figures D2, D4, D6, D8, D10, D14, D16, D18, D20, D22, D24, D26, D30, D32, D34, respectively). In game 2, the fastball pitching velocity over the game remained consistent, with a very slight increase (Figure D3). However, in game 2, the fastball pitching velocity averages over the game remained consistent with a decrease (Figure D4). The reason for the apparent inconsistency in game 2 is because only 3 innings were pitched and the first inning had a slightly higher average than the third inning, which reversed the linear line of best fit. In games 6 and 14, the fastball pitching velocities over the game remained consistent with a very slight increase (Figures D11 and D27, respectively). In games 6 and 14, the fastball pitching velocities averages over the game 
remained consistent with an increase (Figures D12 and D28 respectively). In games 7 and 10, the pitching velocities over the game remained consistent with no visible evidence of any increase or decrease (Figures D13 and D19, respectively).

Average Fastball Pitching Velocity by Subject

Figures D5-D6, D13-D14, D21-D22, D27-D28, and D37-D38 reflected pitches thrown by subject 1. Figures D7-D8, D15-D16, D23-D24, D29, D31, and D39-D40 reflected pitches thrown by subject 2. Figures D9-D10, D17-D18, D25-D26, D33-D34, D35-D36, and D41-D42 reflected pitches thrown by subject 3. Figures D1-D2, D3-D4, D11-D12, D19-D20, D30, D32, and D43D44 reflected pitches thrown by subject 4 .

Subject 1 had the highest overall pitch velocity average throughout the 4 games he pitched, which was $92.45 \pm 0.95 \mathrm{mph}$ (Figure D37). Subject 3 exhibited the second highest overall pitch velocity over a span of 5 games, with an average pitch velocity of $92.06 \pm 1.2 \mathrm{mph}$ (Figure D41). Next was subject 4, with an average pitch velocity of $88.86 \pm 1.0 \mathrm{mph}$ (Figure D43). Finally, subject 2 had the lowest average pitch velocity at $88.05 \pm 1.3 \mathrm{mph}$ (Figure D39). Figures D37 through D44 indicated pitching velocity consistency throughout a season $(91.4 \pm$ 2.8) as opposed to innings throughout a single game. The average pitching velocity over the 4 or 5 games pitched by each subject all trended upward according to the line of best fit, indicating an overall increase in average pitching velocity from the start of the season to the end of the season $(88.05 \pm 1.3 \mathrm{mph}$ to $92.45 \pm 0.95 \mathrm{mph})$. However, each subject had fluctuations in average pitch velocity that indicated a lack of consistency. Subject 1 was the most consistent of the 4 subjects, as indicated by having the lowest standard deviation of 0.95 (Figure D37). One should also consider the number of games played and the effect on pitch velocity variation. Subject 3 pitched 
in five games, while subject 1 pitched in four games, subject 2 pitched in three games in addition to one partial, and subject 4 pitched in four games in addition to one partial.

\section{Use of Blood Flow Restriction}

Following one or multiple pitching bouts, the pitcher would exit the game and not return for the remainder of the game. At this point, unless the player was injured, the head baseball athletic trainer applied a BFR SmartCuff on the proximal portion of the pitching arm and inflated it to a certain pressure, with the intention of occluding blood flow. The cuff was then inflated for five minutes with no concomitant exercise and then deflated for one minute. This process of inflation followed by deflation was repeated three times, for a total treatment duration of 18 minutes, in which, the cuff was inflated for 15 minutes. Deviations in this procedure were due to multiple pitchers leaving the game within a short timeframe (less than 18 minutes apart). Only one SmartCuff device was available to use. Therefore, which pitchers received blood flow restriction and when was unknown. Thus, causality could not be determined whether the consistency in fastball velocity was related to the use of a BFR recovery treatment.

\section{DISCUSSION}

The purpose of this study was to retrospectively investigate the consistency in throwing velocity of fastballs over one competitive baseball season, with four of the starting pitchers undergoing BFR. The hypothesis was that for each subject, there would be a difference in pitching velocity of fastballs when observing the trend throughout the season. This hypothesis was supported in that the pitch velocity slightly increased for each subject as the number of innings played increased. When observing the average pitch velocity by innings played (33 innings for subject 1, 19 innings for subject 2, 24 innings for subject 3, and 19 innings for subject 
4), the linear line of best fit for each subject's pitching average by inning increased. However, different conclusions were reached when observing trends throughout a single game in that average pitch velocity slightly decreased or remained consistent throughout a single game.

As this is the first known study to retrospectively evaluate pitch velocity consistency of four pitchers during the course of a baseball competitive season following BFR recovery treatment, there are very few studies to compare with this study. Also, as variability existed whether a pitcher received a BFR recovery treatment or not, causality could not be determined.

\section{Fastball Pitch Count and Velocity}

The volume of games expected from pitchers requires them not only to be powerful with each pitch, but also have the endurance to pitch multiple innings and maintain longevity throughout the season. ${ }^{13}$ There are many factors involved in fatigue, but in pitching, fatigue can be objectively observed by decreased throwing velocity, decreased accuracy of pitches, increased arm soreness and an increased risk of injury. ${ }^{12}$

Fatigue can demonstrate a performance decline not only throughout one game, but also throughout a season and throughout a career. Maintaining or improving pitch velocity has been a struggle by baseball players at all levels, and many strategies arose to help counter pitching deterioration. The challenge of maintaining pitching velocity over a career was demonstrated by Petti et al. ${ }^{19}$ in which pitch velocity significantly decreased at age 26 , which is relatively young for a professional baseball player. Furthermore, Schulz et al. ${ }^{20}$ found that baseball statistics that indicate success typically weaken after age 27 . Pitch velocity decreasing is not only an implication of declining performance, but can also be linked with a higher rate of injuries. ${ }^{21}$ 
The underlying reason it is so difficult to maintain or improve pitching velocity is because of the kinematic changes that occur with fatigue. When pitching while fatigued, the upper extremity is subject to great stresses, particularly excessive valgus force at the elbow and internal rotation at the glenohumeral joint. ${ }^{22}$ Additionally, there are biomechanical changes that occur in the trunk and lower extremity with fatigued pitching. Essentially, the pitching mechanics become lazy, and lead to increases in stride length, hip flexion, ball release point and hip lean. Furthermore, knee flexion decreases with cocking. ${ }^{21}$ The observations aforementioned were demonstrated in a study by Grantham et al. ${ }^{21}$ that involved collegiate baseball players. The nature of this study was biomechanical and sought to determine if there were deviations in pitching technique associated with increasing fatigue. Using 26 different biomechanical markers, the study found that longer innings (characterized by lasting more than 15 pitches) displayed greater deviations in technique than shorter innings. ${ }^{21}$

Whiteside et al. ${ }^{23}$ conducted a study on Major League Baseball pitchers, observing baseball statistics including pitch type, velocity, ball movement, release point and result of the pitch. These pitching characteristics demonstrated interesting variability throughout nine inning games, particularly those involving pitch type and speed. The number of fastball pitches thrown in the later innings decreased, while the number of pitches such as breaking balls and curve balls increased. ${ }^{23}$ While the current study only investigated fastballs thrown, one can observe parallels between this study and the study by Whiteside et al. ${ }^{23}$ in that, the number of fastballs did decrease in the later innings. The subjects in the study also threw other pitch types (breaking balls and changeups, more specific pitch types were not differentiated), which remained consistent in number throughout the innings pitched. Additionally, the population examined in these two studies are also similar in that they both involve high level, elite baseball players. 
However, the current study investigated four pitchers in a Division I baseball program as opposed to professional MLB players.

Furthermore, Whiteside et al. ${ }^{23}$ found that there were significant decreases in pitch speed found in the fifth inning, which only became more apparent by innings seven through nine. Decreased pitch speed could be intentional or unintentional in nature. ${ }^{14}$ An unintentional cause of lower pitch velocity could be due to the negative effects of fatigue. An intentional source of decreased pitch velocity could be changing the pitch type for strategic purposes. Intentionally slowing down pitches for strategy could be to prevent injury in order increase the pitcher's longevity or it could be a way to play to the opposing team's weaknesses.

However, in the current study, pitch velocity in the majority of games remained relatively consistent (with the exception of only games 6 and 14 where there was an increase in pitch velocity observed). Games 7 and 10 were the most consistent, while games 1-5, 8-9, 11-13 and 15-17 demonstrated an almost completely horizontal line of best fit with either a slight increase (game 2) or a slight decrease (games 1, 3-5, 8-9, 11-13 and 15-17). On the other hand, when each subject was examined individually, their pitch velocity slightly increased on average throughout the duration of the season. There are many factors that can contribute to why these trends were observed, but as the season progressed, the subjects gained experience, which could be attributed to the increased fastball pitch velocity. Additionally, the difficulty of the opponent and the significance of the game could have played a role in better-performing games for the subjects. The majority of the games were conference games and the one that was not a conference game was against a longtime rival school. Finally, the weather getting warmer in the later months of the season could increase performance since warmth is associated with pitching arm "looseness" leading to optimal pitching conditions. It could be postulated that the reason for the increased 
seasonal longevity and pitch velocity increasing as the season progressed could be attributed to the application of BFR as a recovery agent, but further studies are warranted.

Average Fastball Pitching Velocity by Pitcher

Different pitchers have varying strengths and skillsets. Some pitchers specialize in fastball pitching due to their powerful pitching with high velocity. Other pitchers have a different pitching repertoire that involves pitches such as a breaking balls and changeups that are interspersed throughout the inning or game. When these pitches are thrown may be based on the hitter's scouting report or pitches determined by the catcher or pitching coach. Expanding the skillset of a pitcher contributes to their adaptability and ability to be a greater asset to the team as the inning and game progresses. However, the current study focused on pitching performance as it pertained to fastballs. Future studies should focus on other types of pitches to note if variability may also exist.

Subject 3 had the most fastballs pitched (292), while subject 1 pitched for the most innings (33). Fastballs appear to be a strength of subjects 1 and 3 due to the high volume of pitches thrown and innings played in. However, these subjects may be stronger at pitching fastballs due to a limited repertoire of other pitch types that have a lower velocity but require more skill and technique. Additionally, pitching at a maximal speed is not beneficial without accuracy. The current study does not present any strike zone data, so pitch accuracy is unknown, therefore high pitch velocity is not indicative of optimal performance.

Fatigue and the Role of BFR

The biomechanical explanation for decreased pitch velocity is physiological fatigue contributing to sensorimotor changes and impaired performance. ${ }^{14}$ During exercise, the human 
body metabolizes nutrients in order to gain energy. ${ }^{13}$ The manner in which this metabolic reaction occurs can be either aerobic or anaerobic, and the body can switch between the two energy systems. Anaerobic metabolism occurs in the absence of oxygen and exercise in this form requires a greater recovery period post-exercise in order for the body's physiological state to return to baseline. Baseball is a predominantly anaerobic sport, particularly with pitching. ${ }^{13,16,24,25}$ Whenever a pitcher experiences fatigue, it affects the throwing arm significantly. Glenohumeral internal and external rotation can be significantly diminished with repetitive throwing and typically requires ten minutes to recover fully. ${ }^{14,16}$ Other joints associated with baseball pitching include the scapulothoracic and humeroulnar, which respectively require seven and four minutes to recover, as concluded by Livingston et al. ${ }^{16}$ in a study with youth participants.

Decreased performance is not the only effect of fatigue, as fatigue can also contribute to injury. Central nervous system fatigue is associated with general, rather than localized, fatigue, but it can be perceived as a painful experience which can be distracting to an athlete attempting to perform a high intensity skill. ${ }^{24}$ Lack of focus can contribute to improper technique, subsequently leading to injury. Additionally, loss of motor control (another effect of fatigue) can cause an injury to occur. Fastballs typically produce higher injury rates as opposed to other pitch types due to the biomechanical stress that high velocity pitches inflict on the body. ${ }^{16,26}$

Fatigue in baseball is multi-faceted and relies upon many markers. Repetitive rapid force production-based activities, such as pitching, can lead to microtrauma. Microtrauma of the muscles and joints triggers inflammation in the upper extremity each time a pitcher throws the ball. ${ }^{27,28}$ Arm strength (including grip strength) decreased significantly with increased pitching loads, as demonstrated by Sonne et al. ${ }^{27}$ in a study that involved a simulated baseball game with 
MLB players. Other markers include muscle soreness associated with pitching which is a source of painful stimulus, making it difficult to maintain pitching velocity. ${ }^{28}$

Healthy static and dynamic stabilizers contribute to safe pitching practices and reduce the incidence of injury. Both muscles and non-contractile tissue such as ligaments and joint capsules work cohesively to contribute to proper biomechanics. ${ }^{29}$ This process begins with muscle activation, as the dynamic stabilizers can reduce stresses by tensing and decreasing load to other structures such as ligaments and the capsule. ${ }^{27}$ However, less rest time with increased muscular exertion can lead to muscle fatigue. Muscle fatigue inhibits or disrupts muscle activation firing patterns and can decrease joint proprioception. Decreased proprioception is associated with decreased joint stability and capsular laxity which can also lead to injury. ${ }^{29}$ In general, joint kinematics are drastically altered when fatigue sets in and forces are redistributed to tissues that may not be equipped to resist the stress. ${ }^{30}$

Most of the games investigated in the current study did not involve the same pitcher throwing for the entire nine innings. It was observed that the pitching velocity had fluctuations, but slightly decreased as the game progressed. However, the changes demonstrated in this study were somewhat mitigated, which could be attributed to the use of BFR, which was used to assist with recovery. Since this study was not causational, the outcome is purely evidence-based speculation, rather than a direct result of BFR application.

Promoting recovery can also assist in retarding the progress of fatigue. "Recovery" has varying definitions among the literature, but in the context of baseball pitching, recovery is always discussed in relation to fatigue. Any strategies, tools, modalities or other resources that are used to diminish the negative physiological effects of fatigue and return body systems to baseline status are deemed recovery agents. ${ }^{24}$ The concept of recovery, demonstrates that fatigue 
can be diminished in as few as 4 to 7 minutes when using the appropriate methods. ${ }^{14}$ Some methods that were traditionally used for baseball recovery include active recovery (i.e. jogging to gradually reduce heart rate and restore blood flow to extremities), passive recovery (avoidance of physical activity for at least six minutes), or electrical stimulation (which delivers a low electrical current to the muscles exerted). ${ }^{13}$

Recovery agents can benefit performance as they assist with restoring the body's baseline function, optimizing the physical state of an athlete during competition. Baseball athletes rely on many recovery methods in order to perform, which can include postgame recovery as well as between-inning recovery. ${ }^{13}$ For between-inning recovery, the goal is to keep the pitcher in their prime by keeping their pitching arm warm and "loose", as well as maintain adequate blood flow to the pitching arm. ${ }^{13}$ Additionally, electromuscular stimulation, ice, and intermittent compression devices such as Normatec can be useful for postgame recovery. ${ }^{13,31}$ BFR is a newer recovery tool, and has only been studied in the lower extremity, but Cerqueira et al. ${ }^{32}$ found that BFR without concomitant exercise can contribute to increased strength and muscle girth. Strength can decrease when a pitcher becomes fatigued, ${ }^{16}$ so one can infer that increasing strength with passive BFR can assist in resting the pitching arm as well as expediting recovery to return the pitching arm to baseline strength.

What is problematic with collegiate baseball players is that specifically schedules require play up to four games per week, which does not allow adequate recovery time. ${ }^{33}$ In the current study, BFR was used as a means for postgame recovery, as the cuff was only applied to the pitcher once they were finished pitching for the game. Reducing pitcher's fatigue with a fast recovery time can improve performance and decrease chance of injury. ${ }^{13}$ The reason for the increased seasonal longevity and pitch velocity increasing in this current study could be 
attributed to the application of BFR as a recovery agent. However, causality could not be determined between consistencies in throwing velocity while using BFR as a recovery agent. Future studies should retrospectively observe pitching seasons where BFR was not used with a season where it was using the same pitchers from the previous seasons.

\section{Clinical Implications}

Schedules require play up to four games per week, which does not allow adequate recovery time.$^{33}$ Initially, it was thought that rest was the key to combat injury potential. Baseball programs began implementing the 100-pitch count limit to relieve pitchers and reduce overloading, however, injury rates continued to increase. ${ }^{13}$ Furthermore, rest (also known as "passive recovery") in short intervals (likely between innings) causes the heart rate to decrease but does not promote effective blood flow, which is one of the primary goals of recovery. ${ }^{13}$ Additionally, there are significant time restraints associated with baseball pitching. In the MLB, there is a twelve second limit between the time the pitcher receives the ball from the catcher and when they deliver the next pitch. This rule increased the pace of play, also contributing to more influence of fatigue in baseball pitching. ${ }^{27,28}$ Eccentric muscle contractions associated with baseball pitching deceleration places excessive load on the upper extremity which leads to microtrauma then acute fatigue, leading to chronic fatigue and ultimately, injury. ${ }^{34}$ The goal of the BFR intervention as an acute form of recovery in the current study was to reduce chronic fatigue in order to avoid development of negative fatigue effects that put pitchers at risk for injury as the season progresses.

Clinically, the current studied showed promise in terms of application in a Division I baseball program. The data reflected that throughout a season, pitching velocity of fastballs by individual pitchers increased throughout an increasing number of innings pitched, which may 
have been influenced by the application of BFR postgame as a recovery tool. The use of BFR in recovery can be groundbreaking due to the physiological mechanisms behind cellular swelling. Restricting blood flow has the ability to completely change fluid dynamics in cells which stimulates protein synthesis, a key part of recovery. ${ }^{35}$ Replenishing and producing protein essential to tissue healing acutely after being subject to microtrauma that occurs during exercise will be beneficial in the long term. In a study that involved measuring fluid shift with an intervention that involved BFR without concomitant exercise, researchers found a decrease in plasma volume of 15 percent, which explained that cell swelling on the muscular level was due to a plasma fluid shift into the muscle. ${ }^{35}$ This demonstrated that inflating the cuff alone can increase protein synthesis, which can help reduce the microtrauma that is inherent to pitching. ${ }^{35}$

Results demonstrated that the pitchers were fairly consistent, but not all pitched the entire nine-inning game, with the exception of one individual (subject 1). While fastballs were the only data evaluated, other pitch types were thrown. In future studies, examining other pitch types could reveal different or similar trends in not necessarily pitch velocity, but other pitching characteristics such as spin rate and release point. Anecdotally, it was noted that during the postseason, the pitchers demonstrated more consistency and appeared "fresher" than the opposing team pitchers and adapted better than opponents in the post-season. However, to verify the results observed, this observational study should be pursued in future studies and implemented with more control over the variables. BFR demonstrated great benefits in a small subject population that outweigh the rare risks associated with it when applied by a trained individual. The potential to do harm is virtually nonexistent with BFR, which makes it a worthwhile recovery modality.

\section{Limitations}


A major limitation of the current study was that the sample size was small (only four subjects). The study would have been much more generalizable had there been more than four subjects included. Four subjects were not sufficient to be a representative sample of an entire baseball program, which contains over twenty pitchers. Additionally, the data only reflected pitching statistics for home games, which significantly reduced the number of pitches included in analyses. Finally, there was a five-game discrepancy in terms of home games played (22) and games where data was available (17). Data was not applicable or corrupted for five games which also reduces the number of data points, as well as statistical accuracy in the calculations.

Other limitations were inherent to a field study in that there was no available control for comparison. All the subjects included were subject to BFR and the study only included pitching data for one season. If this were done across multiple seasons, some seasons could have served as a control without BFR intervention, or if there were more subjects, some could have undergone BFR intervention, while others utilized other recovery methods.

The BFR protocol followed in the current study has not been evaluated in the literature as a means of enhancing performance. It has, however, been investigated in the literature, in a recovery context in the lower extremity in order to maintain muscle strength and size for those undergoing post-operative protocols. Use of BFR has also been investigated in the ability to increase muscle size and strength in the lower extremity when used in this manner (without concomitant exercise). Additionally, a biomechanical analysis did not occur to see if there were alterations in the throwing patterns for each subject throughout the season.

\section{CONCLUSION}


Although there have been numerous studies utilizing orthodox BFR protocols with exercise on an athletic population, this is the first study that descriptively investigated BFR without concomitant exercise in a field study on an athletic population. BFR in a recovery context is relatively new, particularly with baseball pitchers. The current study found that pitch velocity of fastballs remained consistent with game progression and throughout the season. BFR can be a recovery tool to diminish the negative effects of fatigue on pitch velocity and overall pitching performance, contributing to a consistent pitcher. 


\section{REFERENCES}

1. Shinohara M, Kouzaki M, Yoshihisa T, Fukunaga T. Efficacy of tourniquet ischemia for strength training with low resistance. Eur J Appl Physiol. 1998; 77(1):189-191.

2. Head P, Austen B, Browne D, Campkin T, Barcellona M. Effect of practical blood flow restriction training during bodyweight exercise on muscular strength, hypertrophy and function in adults: a randomised controlled trial. Int J Ther Rehabil. 2015; 22(6):263271.

3. Hughes L, Paton B, Haddad F, Rosenblatt B, Gissane C, Patterson SD. Comparison of the acute perceptual and blood pressure response to heavy load and light load blood flow restriction resistance exercise in anterior cruciate ligament reconstruction patients and non-injured populations. Phys Ther Sport. 2018; 33:54-61.

4. Yow BG, Tennent DJ, Dowd TC, Loenneke JP, Owens JG. Blood flow restriction training after achilles tendon rupture. J Foot Ankle Surg. 2018; 57(3):635-638.

5. Loenneke JP, Allen KM, Mouser JG, et al. Blood flow restriction in the upper and lower limbs is predicted by limb circumference and systolic blood pressure. Eur J Appl Physiol. 2015;115:397-405.

6. Giles L, Webster KE, McClelland, Cook JL. Quadriceps strengthening with and without blood flow restriction in the treatment of patellofemoral pain: a double-blind randomised trial. Br J Sports Med. 2017; 51(23):1688-1694.

7. Iversen E, Rostad V, Larmo A. Intermittent blood flow restriction does not reduce atrophy following anterior cruciate ligament reconstruction. J Sport Health Sci. 2016; 5(1):115-118.

8. Cerqueira MS, Vieira WHB. Effects of blood flow restriction exercise with very low load and low volume in patients with knee osteoarthritis: protocol for a randomized trial. Trials. 2019;20(135):1-9.

9. Vopat BG, Vopat LM, Bechtold MM, Hogde KA. Blood flow restriction therapy: where we are and where we are going. J Am Acad Orthop Surg. 2019;00:1-8.

10. Manimmanakorn A, Manimmanakorn N, Taylor R et al. Effects of low-load resistance training combined with blood flow restriction or hypoxia on muscle function and performance on netball athletes. J Sci Med Sport. 2013;16(4):337-342.

11. Scott BR, Loenneke JP, Slattery KM, Dascombe BJ. Blood flow restricted exercise for athletes: a review of available evidence. J Sci Med Sport. 2016;19: 360-367. 
12. NCAA. Division I baseball. https://www.ncaa.com/sports/baseball/d1. Accessed 5 October 2019.

13. Warren CD, Brown LE, Landers MR, Stahura KA. Effect of three different betweeninning recovery methods on baseball pitching performance. J Strength Cond Res. 2011;25(3):683-688.

14. Tripp BL, Yochem EM, Uhl TL. Functional fatigue and upper extremity sensorimotor system acuity in baseball athletes. J Athl Train. 2007;42(1):90-8.

15. Potteiger JA, Lockwood RH, Haub MD, et al. Muscle power and fiber characteristics following 8 weeks of plyometric training. J Strength Cond Res. 1999;13(3):275-279.

16. Livingston JL, Tavoukjian NM. Lower extremity strength and recovery time in youth baseball pitchers: a pilot study. J Strength Cond Res. 2018:1-32.

17. Trackman. baseball.trackman.com. Accessed 15 September 2019.

18. Pexa BS. The influence of training load on musculoskeletal injury risk variables, objective fatigue, subjective well-being, and performance in baseball athletes. Sport Med. 2019:1-236.

19. Petti, B. (2012, April 30). Pitcher aging curves: Introduction. Retrieved April 27, 2020, from http://www.fangraphs.com/blogs/pitcher-aging-curvesintroduction/

20. Shulz R, Musa D, Staszewski J, Siegler RS. The relationship between age and major league baseball performance: implications for development. Psychol Aging. 1994;9(2):274-286.

21. Grantham WJ, Byram IR, Meadows MC, Ahmad CS. The impact of fatigue on the kinematics of collegiate baseball pitchers. Orthop J Sports Med. 2014;2(6):1-10.

22. Kibler WB, Sciascia A, Moore S. An acute throwing episode decreases shoulder internal rotation. Clin Orthop Relat Res. 2012;470(6):1545-1551.

23. Whiteside D, Martini DN, Zernicke RF, Goulet GC. Changes in a starting pitcher's performance characteristics across the duration of a major league baseball game. Int $J$ Sports Phys Perf. 2016;11(2):247-25. 
24. Zajac A, Chalimoniuk M, Maszczyk A, Golas A, Lngfort J. Central and peripheral fatigue during resistance exercise - a critical review. J Hum Kinet. 2015;22(49):159-169.

25. Freeston J, Adams R, Ferdinands RED, Rooney K. Indicators of throwing arm fatigue in elite adolescent male baseball players: a randomized crossover trial. J Strength Cond Res. 2014;28(8):2115-2120.

26. Birfer R, Sonne MW, Holmes MW. Manifestations of muscle fatigue in baseball pitchers: a systematic review. PeerJ. 2019; 29(7):7390.

27. Sonne MW, Keir PJ. Major league baseball pace-of-play rules and their influence on predicted muscle fatigue during simulated baseball games. J Sports Sci. 2016;34(21):2054-2062.

28. Yang SC, Wang CC, Lee SD, et al. Impact of 12-s rule on performance and muscle damage of baseball pitchers. Med Sci Sports Exerc. 2016;48(12):2512-2516.

29. Fleisig GS, Andrews JR, Dillman CJ, Escamilla RF. Kinetics of baseball pitching with implications about injury mechanisms. Am J Sports Med. 1995;23(2):233-239.

30. Escamilla RF, Barrentine SW, Fleisig GS et al. Pitching biomechanics as a pitcher approaches muscular fatigue during a simulated baseball game. Am J Sports Med. 2007;35(1):23-33.

31. Keck NA, Cuddy JS, Hailes WS, Dumke CL, Ruby BC. Effects of commercially available pneumatic compression on muscle glycogen recovery after exercise. $J$ Strength Cond Res. 2015;29(2):379-385.

32. Cerqueira MS, Nascimento JD, Maciel DG, Barboza JAM, Vieria WH. Effects of blood flow restriction without additional exercise on strength reductions and muscular atrophy following immobilization: a systematic review. J Sport Health Science. 2019;1-9.

33. Trends year by year. http://fs.ncaa.org/Docs/stats/baseball_RB/reports/TrendsYBY.pdf. Accessed 5 October 2019.

34. Magnusson SP, Gleim GW, Nicholas JA. Shoulder weakness in professional baseball pitchers. Med Sci Sports Exerc. 1994;26(1):5-9.

35. Owens J. Blood flow restriction rehabilitation. www.owensrecoveryscience.com. Accessed 5 September 2019. 


\section{APPENDICES}




\section{APPENDIX A}

\section{THE PROBLEM}

\section{Research Question}

On average, there are 54 regular-season games for Division-I collegiate baseball. ${ }^{33}$ This contrasts with basketball (31 games on average) and football (12 games on average), which are considered other top-tier collegiate sports. ${ }^{12}$ However, the demand placed on baseball players, particularly pitchers, is substantially different than football or basketball. Baseball pitchers are expected to perform consistently and frequently, on an individual basis, despite the concept of a team. Since the demand placed on baseball pitchers is unique to any other sport, the training regimen should also be unique in order to improve performance.

Performance enhancement training has a focus in increasing strength and hypertrophy, but also in maintenance and injury prevention. The injury prevention component is primarily focused on combating fatigue. In pitchers, the sheer volume of innings pitched, and number of pitches leads to fatigue. Fatigue is a physiological state that is due to a decrease in blood flow, however, blood flow is required to replace glycogen stores in the liver. ${ }^{13}$ Fatigue can also decrease acuity in pitchers at the glenohumeral, scapulothoracic and wrist joints. ${ }^{14}$ Ultimately, fatigue can decrease focus, motor skills and strength which can contribute to increased chance of injury. ${ }^{13}$ Pitching is considered a high-intensity motion, which baseball players perform repeatedly, that leads to an increase in lactic acid in blood circulation of the upper extremity, which is undergoing the greatest stresses. ${ }^{13}$ Lactic acid accumulation decreases $\mathrm{pH}$ and available oxygen in the muscle. Homeostatic disruptions, such as changes in $\mathrm{pH}$, creates a non-optimal environment for muscle function that leads to motor impairment and negatively affects pitching 
performance. ${ }^{15}$ However, since this non-optimal physiological environment created in the muscle is inevitable, then baseball players should be training in a context that mimics the physiological state that occurs during long bouts of pitching. ${ }^{13}$

Blood flow restriction (BFR) training has recently been introduced as a rehabilitation tool, but was initially used among the strengthening community (i.e. competitive weight lifting, body building, etc.). ${ }^{1}$ BFR is characterized by temporary ischemia following application of a cuff device that has a similar effect to a tourniquet. ${ }^{1-3}$ There are various cuffs mentioned throughout the literature, but all are designed to cut off or occlude some transport of blood throughout vascular structures (arteries, veins, arterioles, venules, capillaries). As BFR is designed for use on the extremities, the cuffs are applied to the proximal portion of the targeted limb. Examples of the cuffs include a pneumatic cuff, ${ }^{2,3}$ tourniquet ${ }^{2,3}$ or wrap sometimes known as a KAATSU device when manufactured by that company. ${ }^{1}$ The ischemic effect of the cuff device is achieved by the fulfillment of two criteria: full occlusion of venous drainage and partial occlusion of arterial blood flow. Restricting 100 percent of venous drainage is completed by prohibiting blood movement away from an extremity. ${ }^{1-3}$ Occlusion pressure when applying BFR to the upper extremity is typically about 50 percent of an individual's systolic pressure, while it is about 80 percent of that systolic pressure in the lower extremity. ${ }^{35}$ Prolonged occlusion of blood vessels, particularly arterial structures, can lead to permanent cardiovascular or musculoskeletal damage, which is why close monitoring during application is important. ${ }^{2,36}$

BFR use in a performance context is a relatively recent concept. However, BFR previously demonstrated the ability to increase muscle adaptations such as strength and hypertrophy in many contexts. ${ }^{9} \mathrm{BFR}$ not only produces muscle adaptations comparable to traditional high resistance training, but also simulates a muscle environment with decreased 
oxygen available due to blood occlusion (which mimics the fatigued pitching arm). Low-load BFR training is beneficial in supplementing sport-specific conditioning and workouts and presents a decreased risk for injury occurrence because the external load is minmized. ${ }^{9}$ The observed increase in strength and muscle mass can translate into sport-specific, functional activities such as 5 meter sprint, 20 meter shuttle run and 505 agility. ${ }^{10}$ While upper extremity information is limited as compared to lower extremity studies, a 4-week BFR training protocol demonstrated an increase in upper and lower chest girth as well as a 7 percent increase in a 1 repetition maximum (1RM) for bench press. ${ }^{10}$

The implications of BFR are useful in many settings, but particularly in an athletic context. Due to the prevalence of injury and the unique nature of pitching, BFR could be useful in supplementing athletic practices and lifting sessions to enhance performance. With a strict protocol as well as close monitoring, BFR can be implemented safely and effectively. More research is needed to determine the long-term effects of BFR throughout a baseball season to observe any change in pitching performance. Based on the use of BFR with baseball, the following research question is asked: Will pitch velocity remain consistent throughout a competitive season following blood flow restriction?

Experimental Hypothesis:

1. For each subject, there will be a difference in pitching velocity of fastballs as observed throughout home games during one competitive baseball season.

Assumptions

1. TrackMan data provided valid and reliable data regarding pitch type and pitch velocity.

2. The four pitchers engaged in BFR during the competitive season. 
3. The BFR protocol used was consistent throughout the competitive season.

4. The individual implementing BFR was trained and experienced in BFR application.

\section{Delimitations}

1. Subject population was limited to four male pitchers at one mid-Atlantic university. The results will not be generalizable to other collegiate baseball pitchers.

2. Subject population was limited to starting baseball pitchers that competed on a team at one mid-Atlantic university during fall 2018 to spring 2019.

\section{Operational Definitions}

1. Baseball season- The duration of the season is the competitive baseball season once competition begins against other universities whether conference or non-conference.

2. Blood occlusion- Venous blood flow ceases and arterial blood flow partially ceases to the affected extremity. ${ }^{35}$

3. BFR- Blood flow restriction; Temporary partial ischemia to an extremity that occurs following application of a tourniquet device. ${ }^{1}$ This requires close monitoring and training by the individual applying the device. A low-load exercise routine is conducted while the device is occluding blood flow to the extremity. Strength and hypertrophy are increased by BFR application. ${ }^{2}$

4. BFR collection period- Implemented during the baseball season during fall 2018-spring 2019.

5. BFR cuff pressure- For the upper extremity, the cuff should be inflated so that it is 50 percent of the individual's systolic blood pressure, indicating occlusion is adequate and safe. $^{35}$

6. Fastball- A pitch thrown at approximately a pitcher's maximum speed.

7. Fatigue- An internal physiological state, which can result from high volumes of pitching leading to a decrease in oxygen available from decreased blood flow. This can lead to decreased performance (by decreasing strength and coordination) and increased chance of injury. ${ }^{13}$

8. Low-load training- Used in conjunction with BFR application, this can result in hypertrophic and strength gains. ${ }^{1}$ Typically, low-load training requires a longer duration in order to observe muscle adaptations. Low-load exercise is designed for patients that 
may have a lower tolerance threshold for exercise, and eventually is increased to a higher load as exercise tolerance increases.

9. Performance- Measured by the outcome of pitching velocity, many factors can influence performance. Fatigue can decrease performance, while strength and neuromotor control can increase performance as measured by pitch speed. ${ }^{13}$

10. Pitching velocity- The speed of a fastball pitch (in miles per hour), as measured by the TrackMan radar.

11. Smartcuff- The tourniquet device used to apply BFR in this study. The cuff is placed at the proximal portion of the upper extremity and inflated to an individualized pressure, with the intention of occluding arterial blood flow by 50 percent. ${ }^{35}$

12. TrackMan- A technology that records ball tracking during games. It incorporates military-grade 3D Doppler radar to track both pitching and hitting data based off the Cartesian location at home plate and involves a large radar panel installed behind home plate. Statistics tracked includes pitch velocity, spin rate, tilt, extension, effective velocity, zone velocity, release height, release side, vertical break, horizontal break, exit speed, launch angle, direction, spin rate, hang time, bearing, and distance. ${ }^{17}$

Limitations

1. This study has low external validity; is not generalizable to other baseball pitchers from other Division I universities or other competitive levels.

2. BFR cuff pressure is not standardized, but instead is based on the size of the individual's extremity.

3. BFR implementation schedule is not standardized.

Significance of the Study

There are hundreds of D1 collegiate baseball players, that play an average of 54 games per season. ${ }^{33}$ Pitchers throw countless balls leading to fatigue and potentially, decreased motor ability which can contribute to poor mechanics and, in turn, injury. ${ }^{13,14,15}$ BFR could be a tool that promotes metabolic changes in the muscle, allowing for reduced injury rates and improved 
performance. ${ }^{1}$ BFR has already proven useful as a rehabilitation tool, but implications of BFR without concomitant exercise ${ }^{8}$ and BFR as a performance tool is relatively in the infancy stages.

One study has demonstrated that BFR can increase an athlete's 1RM in bench press, but further studies need to be conducted to determine if more sport-specific activities can improve. Additionally, longitudinal effects of BFR throughout an entire baseball season need to be observed to see if there are fluctuations in performance trends. This study sought to retrospectively investigate throwing velocity of fast balls to see if BFR implementation had any influence throughout a baseball season with BFR. Demonstrating quantitative changes such as throwing velocity could reinforce the importance of BFR to coaches and athletes to promote increased adherence to the BFR protocol. This information can be disseminated to athletic trainers and pitching coaches at professional conferences and workshops. 


\section{APPENDIX B}

\section{LITERATURE REVIEW}

Introduction

Traditionally, resistance training is known for increasing strength and hypertrophy. Resistance training is characterized by exercise performed with some weight additional to gravity at an intensity that is at least 60 to 70 percent of the individual's maximal force required to complete a movement, termed "one-repetition maximum" (1RM). ${ }^{1-3}$ Examples of weight application in resistance training could include body weight exercises, cuff weights, dumbbells, kettle bells, bars, exercise bands, and more. Clinicians often follow high intensity or heavy load resistive training principles, but recently some clinicians chose to implement blood flow restriction (BFR) training in rehabilitation programs in place of traditional resistance training. ${ }^{3}$

BFR is designed to occlude some transport of blood throughout vascular structures. The Delfi system is the gold standard of BFR according to Owens Recovery Science. ${ }^{35}$ BFR is designed for use on the extremities, so the cuffs are applied to the proximal portion of the targeted limb. Blood flow facilitates the distribution of nutrients and oxygen throughout the body. Therefore, without adequate blood flow, oxygen is scarce, and metabolites accumulate in the affected extremity. Creation of this anaerobic environment within a limb is the main objective of BFR as the metabolic and hormonal changes promote accelerated muscle fatigue via the neuromuscular system. ${ }^{1,2,36}$ Muscle adaptations from neuromuscular fatigue is important because it is the source of strength gains and hypertrophic changes in resistance training. ${ }^{35}$ Highintensity resistance training causes muscle fatigue to occur rapidly (typically when generating a large force required to move a certain volume of weight), whereas low-load training does not typically result in fatigue to muscle failure without excessive durations or repetitions. However, 
low intensity training when combined with BFR demonstrates increases in hypertrophy and muscular strength, sometimes greater than high intensity resistance training. ${ }^{3,36-42}$ BFR increasing strength can also lead to enhancement of power and endurance, which can assist in improving performance. ${ }^{11}$ The use of BFR is evident in the literature for hypertrophy and muscular strength, but limited in performance enhancement. Thus, topics that will be discussed throughout the body of this literature review are as follows: BFR background, physiology behind BFR, use of BFR in the extremities, use of BFR in athletic performance training, safety concerns, baseball, fatigue and recovery as well as BFR without concomitant exercise.

\section{BFR Background}

The primitive form of BFR utilized for KAATSU training was developed in Japan. This use of BFR was indicated for individuals experienced in heavy resistance weight training. Bodybuilders would use BFR at a low load to supplement a heavy weight-lifting regimen. As BFR gained popularity in the strengthening community, applications in the military setting were developed, which was then introduced to rehabilitation for non-military patients. ${ }^{1}$

Approximately 48 million people undergo surgical procedures annually. ${ }^{34}$ Most surgical procedures are somewhat invasive and even with technological advances such as arthroscopy, incisions are required. ${ }^{39,40}$ While surgeries are designed to improve the body's function, the initial aftermath results in adverse effects to the musculoskeletal system (regardless of which body structure was operated on). Many patients are in pain following surgery and are advised to rest to allow the tissues to heal. The post-operative phase often includes some period of bed rest, activity restriction and/or immobilization. During prolonged recovery, patients experience muscle atrophy and weakness due to inactivity. To counter this decrease in strength and muscle mass, patients are required to complete a rehabilitation program following the resting phase. The 
goal of this rehabilitation is to gradually increase the patients' intensity of exercise to regain strength and ability to perform activity at a pre-operative level. ${ }^{1-4,38}$

Muscle weakness can hinder individuals from returning to activity, which is why rehabilitation targets muscle hypertrophy and restoration of function. ${ }^{1,2}$ The implications of BFR are beneficial to populations in which traditional resistance training is not indicated due to a recent operation or other pre-existing health conditions. ${ }^{1,2,4}$ Some of these conditions restrict the patients' activity level because the body lacks protective mechanisms from high-impact forces. ${ }^{3}$ An anterior cruciate ligament (ACL) reconstruction is one of the most prevalent injuries in the literature for post-operative uses of BFR. Additionally, some research surrounds Achilles tendon repair as well as patellofemoral pain and limb amputations. ${ }^{5}$ Other main conditions mentioned in the literature include osteoarthritis (OA),${ }^{1,5}$ sarcopenia,,${ }^{5}$ and rheumatoid arthritis. ${ }^{5}$ Patients who were recently operated on also have physical limitations. ${ }^{1} \mathrm{OA}$ is of particular concern because it is a common source of pain in geriatric populations, as 40 percent of individuals over the age of 60 suffer from this. ${ }^{44}$ Unfortunately, for patients with OA, strengthening is often indicated to reduce disability, but traditional resistance training produces intolerable pain. OA is common in the tibiofemoral joint, which leaves patients with inhibited knee extensor muscles (quadriceps). ${ }^{8}$ Strengthening of the quadriceps (as well as other proximal musculature) would reduce pain and disability, except that resistance training is typically performed with loads greater than 60 percent of a 1 repetition maximum, which can create swelling and inflammation in the knee. Additionally, the pain associated with this type of training is likely to reduce patient participation in exercises. ${ }^{8}$

BFR serves as a safer alternative to high intensity training because there are fewer mechanical forces applied to the joints..$^{1,2,38,40,41}$ In OA, BFR not only has an ability to improve 
function and strength, but also to reduce pain. ${ }^{44}$ Using BFR as a rehabilitation technique allows patients to return to strength training earlier than they could with heavy load exercise, which is a crucial tool in post-operative recovery as well as both prevention and treatment of OA. BFRinfluenced rehabilitation methods can contribute to improved outcomes, prognosis and long-term effects of surgery. ${ }^{38,40}$

In a study by Ferraz et al. ${ }^{44}$, patients with OA performed leg press and knee extension exercises. The study consisted of all women, which were randomized to one of three intervention groups. One group performed low intensity resistance training with BFR (4 sets of 15 repetitions at 20 percent of 1 repetition maximum), another performed low intensity resistance training (without BFR) and the other performed high intensity resistance training (4 sets of 10 repetitions at 50 percent 1 repetition maximum). Both low intensity resistance with BFR and high intensity resistance demonstrated significant improvements during the functional tests (to measure quadriceps muscle strength, function and muscle mass) as opposed to low intensity resistance (without BFR). Additionally, the patient-oriented outcomes showed a decrease in pain with low intensity resistance with BFR. ${ }^{44}$

Other populations who may or may not be recovering from surgery include patients that do not benefit from traditional rehabilitation (due to chronic conditions), active populations (military and athletic) and patients in which $\mathrm{OA}$ is already present. ${ }^{3,40}$ The greatest consequence of surgery is the period of rest following, which requires typically independent individuals to depend on caretakers during recovery. Unfortunately for athletic and military patients, full mobility is required for job responsibilities and being limited in physical activity is not beneficial. Populations such as these are inclined to delay much-needed surgical intervention, which can increase the extent of damage to the injured tissue. Patients in the active population 
complete a rehabilitation program which is slightly altered from the standard progression. As mentioned, all patients undergo a resting period followed by a standard rehabilitation, but active populations are also required to complete functional exercises for clearance to return to daily job tasks. ${ }^{5}$ Military personnel and athletes need to engage in activity-specific rehabilitation to ensure full functionality prior to returning to full participation. Assessing functional strength and movement of an active patient is important in both combat and in sport for maximal performance and to reduce the risk of reinjury. According to the literature, BFR can enhance the efficiency of recovery. BFR's involvement in recovery duration is to minimize the time these patients have to be "out" for, or to be unable to fully participate in activities required for their role. ${ }^{39}$

Physiology Behind BFR

Understanding the physiological mechanisms that make BFR effective by applying low mechanical stress on the musculoskeletal system is essential prior to implementation in rehabilitation. In a landmark study where the researchers investigated an early form of BFR, known specifically as "tourniquet ischemia", an 8-centimeter-wide tourniquet was the style of cuff used to occlude blood flow to the leg and included 5 male patients in a pilot study. Furthermore, the application of the tourniquet required $250 \mathrm{mmHg}$ to create ischemia. Knee extension exercises were performed at 40 percent of the patient's 1RM. The results of the study demonstrated that BFR as an intervention tool increased both torque development and leg strength (maximum voluntary contraction) after 4 weeks of training. ${ }^{1}$ Shinohara et al. ${ }^{1}$ discussed the low mechanical stress placed on the musculoskeletal system, but the neuromuscular system was fatigued due to lack of oxygen. Other studies were consistent with the finding that stressing the neuromuscular system resulted in similar strength gains as seen with high resistance training..$^{2-4,6,36-40}$ 
The physiology of the observed strength increase begins with the accumulation of phosphates and lactic acid, which causes a $\mathrm{pH}$ change in the muscle, indicating the transition of an aerobic to anaerobic environment. ${ }^{45}$ This $\mathrm{pH}$ difference is attributed to the increase in $\mathrm{H}^{+}$ions as a product of metabolism. ${ }^{45}$ Additionally, afferent nerve fibers located in the muscles stimulate the pituitary gland to produce insulin-like growth factor 1 , which when released results in hypertrophy as well as strength increase. ${ }^{1}$ The anaerobic environment of the muscle has been demonstrated by Conceicão et al. ${ }^{48}$ in a study where plasma lactate concentration was significantly higher during exercise and post-exercise in subjects that performed low intensity exercise with BFR than those that performed low intensity exercise without BFR. During this state of anaerobic metabolism, venous return is decreased and elevates metabolic demand, causing cellular swelling which also stimulates hypertrophy. ${ }^{35,48}$ The body attempts to compensate for this state of localized hypoxia by increasing cardiac output, heart rate and ventilation (as measured by carbon dioxide output) to oxygenate the extremity while forcing out metabolites (i.e. phosphates). ${ }^{48}$ The mechanism by which BFR stimulates muscle hypertrophy is by the influx of amino acids into cells as a result of increased stress on muscle cells. ${ }^{47}$ This amino acid accumulation leads to increased production of actin and myosin, which are necessary proteins for muscle contractions to occur. Increased actin and myosin allows for more myofibrils to develop, and the thickness of myofibrils to increase, thus increasing muscle size. ${ }^{47} \mathrm{BFR}$ has also demonstrated cardiorespiratory benefits via metaboreflex and an increase in $\mathrm{VO}_{2 \max }{ }^{48}$ The consensus among researchers is that high resistance training is not the only method to achieve muscle adaptations, it is the fatigue-related processes that occur with exercise. ${ }^{1,2,36,38,41}$

While exercise can occur in an aerobic or anaerobic manner, fatigue is a physiological state that is due to a decrease in blood flow and subsequent lack of oxygen. ${ }^{13}$ Fatigue can 
negatively affect both the neurological system as well as the musculoskeletal system and their junction. In the upper extremity, the glenohumeral, scapulothoracic and wrist joints can be debilitated by fatigue and can cause injury rates to increase. ${ }^{13,14}$ Repetitive, high-intensity motions result in lactic acid accumulation localized to the blood circulation in the involved extremity which, in turn decreases $\mathrm{pH}$ and available oxygen. ${ }^{13}$ Factors that negatively affect homeostasis, such as changes in $\mathrm{pH}$, create a non-optimal environment for muscle function that leads to motor impairment. ${ }^{15}$ However, in a sports context, particularly with baseball, this nonoptimal environment is inevitable. Therefore, baseball players should be training in a context that mimics the physiological state that occurs during long bouts of pitching. ${ }^{13} \mathrm{BFR}$ in a baseball context will be elaborated on under Baseball, Fatigue and Recovery.

Use of BFR in the Lower Extremity

For the lower extremity, there are a plethora of studies ${ }^{2-4,6,7}$ involving BFR, particularly with post-operative ACL care. There is no standardization among the research as it pertains to protocols and Table B1 reflects the major discrepancies in terms of occlusion pressure, continuous versus intermittent application and duration of exercise program. There are a variety of exercises clinicians can apply BFR to including leg press, leg extension, squat ${ }^{2,3,6,7}$ and ankle dorsiflexion and plantarflexion. ${ }^{4}$

Table B1. Studies on BFR Based on Lower Extremity Exercise Programs.

\begin{tabular}{|c|c|c|c|c|}
\hline $\begin{array}{l}\text { Study } \\
\text { Authors }\end{array}$ & Study Participants & Instruments Used & Intervention & Outcome Measures \\
\hline $\begin{array}{l}\text { Head et } \\
\text { al. }{ }^{2}\end{array}$ & $\begin{array}{l}12 \text { participants ( } 4 \text { males, } \\
8 \text { females) healthy adults } \\
\text { (mean age of } 26 \text { years in } \\
\text { the BFR group and } 24.8 \\
\text { years in the control) }\end{array}$ & $\begin{array}{l}\text { Goniometer, } \\
\text { elastic wrap was } \\
7.62 \text { centimeters } \\
\text { in width. }\end{array}$ & $\begin{array}{l}\text { The wrap was tightened at } \\
\text { the proximal thigh until the } \\
\text { patient perceived its' } \\
\text { tightness at a } 7 \text { out of } 10 . \\
\text { Duration of the exercise } \\
\text { program was } 6 \text { weeks, with } \\
\text { participants performing } \\
\text { bodyweight exercises twice }\end{array}$ & $\begin{array}{l}\text { Peak torque for } \\
\text { isometric, concentric } \\
\text { and eccentric exercise, } \\
\text { single leg vertical } \\
\text { jump and thigh girth. }\end{array}$ \\
\hline
\end{tabular}




\section{Hughes 30 participants including et al. ${ }^{3} \quad$ healthy males as well as males that underwent an ACLR.}

\section{Giles et $\quad 79$ participants (87 al. ${ }^{6} \quad$ percent retention, resulting in 69 participants seeing through the entire study), with patellofemoral pain.}

\begin{tabular}{|c|c|c|}
\hline $\begin{array}{l}\text { Iversen } \\
\text { et al. }{ }^{7}\end{array}$ & $\begin{array}{l}24 \text { participants ( } 14 \text { men, } \\
10 \text { women) that } \\
\text { underwent an } \\
\text { arthroscopic anterior } \\
\text { cruciate ligament } \\
\text { reconstruction (ACLR) } \\
\text { with a hamstring tendon } \\
\text { graft that were between } \\
18 \text { and } 40 \text { years old } \\
\text { (mean age } 24.9 \text { for the } \\
\text { BFR group and } 29.8 \text { for }\end{array}$ & $\begin{array}{l}\text { Pneumatic } \\
\text { occlusion cuff } \\
\text { Delphi (14 } \\
\text { centimeter-width } \\
\text { cuff), a portable } \\
\text { blood pressure } \\
\text { pump (Trigger } \\
\text { Aneroid), MRI. }\end{array}$ \\
\hline
\end{tabular}

Unspecified pneumatic cuff per week. Single leg squat was performed to 75 or 90 degrees of knee flexion (a metronome was used for pacing) until fatigue, with one minute in between sets. 3 groups: 1 healthy group that performed BFR exercise, 1 injured group that that performed BFR exercise at a light load and 1 injured group that performed heavy load traditional resistance exercise. The BFR implementation used 80 percent of the patient's limb occlusive systolic pressure. Exercise was also performed at 30 percent of 1 repetition maximum. A warm-up was implemented prior to beginning BFR exercise.

ACUSON color
doppler
ultrasound was
used to locate the
dorsal pedal
pulse,
dynamometer,
pneumatic cuff
(brand and cuff
dimensions not
identified).

2 groups: a standard resistance exercise group (that performed exercises at 70 percent of the 1 repetition maximum with no BFR implementation) and the BFR group (that performed exercises at 30 percent of the 1 repetition maximum). Patients' were supine (with the cuff applied to the proximal thigh) and the cuff was inflated to 60 percent of total dorsal pedal (a maximum pressure of $150 \mathrm{mmHg}$ ). The exercise program was 8 weeks long, 3 times per week and consisted of leg press and leg extension. 2 groups: intermittent BFR and a control group. For intermittent BFR, the cuff was inflated for 5 minutes and deflated for 3 minutes. The initial pressure for all subjects was $130 \mathrm{mmHg}$ with an increase of $10 \mathrm{mmHg}$ each day up until $180 \mathrm{mmHg}$. The exercise protocol was the same for
Pain scale report, ratings of perceived exertion.

Kujala Patellofemoral Score, Visual Analog Scale (for worst pain and for pain with activities of daily living such as squatting, going down stairs and after sitting for 30 minutes), isometric knee extensor torque, quadriceps muscle thickness and global perceived outcome (at a 6-month follow-up).

Swelling, knee mobility, anatomical cross-sectional area of the quadriceps muscles. 
the control group). All subjects were active individuals that were injured during sports participation.

Yow et Patient 1: A 29-year-old al. ${ }^{4} \quad$ male soldier with a left Achilles tendon rupture that was repaired operatively. The patient had complications with wound infections and had a secondary operation for wound debridement. He had functional deficits with plantarflexion and dorsiflexion and used a cane for ambulation.

Patient 2: A 38-year-old male soldier with a right Achilles tendon rupture initially that was repaired operatively, followed by a left Achilles tendon rupture that occurred 8 months post-operatively (for the contralateral side), which the patient opted to treat conservatively.

Delfi PTSii device (14 centimeterwidth cuff).

The study by Iversen et al. ${ }^{7}$ began only 2 days post-operatively, which is an early implementation of exercise in an injured population compared to the other studies examined. The case studies outlined by Yow et al. ${ }^{4}$ (in which BFR exercise began 6 months post-operation for patient 1 and 4 months post-injury for patient 2) and by Giles et al. ${ }^{6}$ examined patients that were also injured, while the other studies used healthy subjects. ${ }^{2,3}$ The research indicated that following ACL surgery, the quadriceps are the primary muscles that are subject to disuse atrophy 
as opposed to the hamstrings or adductor muscles, which are also affected during the unloading or non-weight bearing period. ${ }^{7}$ Additionally, loss of muscle mass occurs more rapidly following arthroscopy than other surgical methods. ${ }^{7}$ However, one potential benefit of using BFR during post-operative rehabilitation is that it can lead to accelerated protein synthesis due to metabolic changes induced by BFR. Metabolites involved in BFR may promote faster healing of tissues involved as well as skin lesions that may occur as a complication from wound closure. ${ }^{4}$ The findings by Yow et al. ${ }^{4}$ are significant because restoring a patient's abilities at the pre-injury level can be an extensive process, with symptoms or deficits present up to 2-year post-initial injury. Hughes et al. ${ }^{3}$ concluded that higher occlusive pressures result in greater metabolic changes within the extremity leading to higher perceptual responses such as pain. This fact was supported by study results that muscle pain was statistically significant between groups, with the BFR (ACL reconstruction) group having the highest average. ${ }^{3}$ However, for intermittent BFR application, Iversen et al. ${ }^{7}$ did not detect any statistically significant changes in quadriceps muscle size.

Many different procedures are used to establish an appropriate occlusion pressure. In many cases, the pressure was individualized to both the patient and the limb (i.e. arm versus leg) based on the patient's systolic pressure..$^{2-4,6,7}$ Additionally, there were differences among the protocols and exercise duration used in each study. Both intermittent and continuous BFR were used and, in some cases, were compared to a control that used traditional resistance training methods. ${ }^{2-4,6,7}$ The patients' power and muscle strength increased exponentially (up to 4475 percent) throughout the treatment period. ${ }^{4}$ Use of BFR in the Upper Extremity 
BFR research in the upper extremity is limited but has seen some benefits. Table B2

outlines two studies that involved upper extremity exercises in order to observe the effects of

BFR. Anecdotally, there are clinicians using BFR to strengthen the dynamic elbow stabilizers for

ulnar collateral ligament pathologies. Upper extremity studies on BFR mainly focus on the

shoulder girdle but could also be expanded to the elbow and wrist/hand. ${ }^{36,42}$

Table B2. Studies on BFR Based on Upper Extremity Exercise Programs.

\begin{tabular}{|c|c|c|c|c|}
\hline $\begin{array}{l}\text { Study } \\
\text { Authors }\end{array}$ & Study Participants & Instruments Used & Intervention & Outcome Measures \\
\hline $\begin{array}{l}\text { Brandner } \\
\text { et al. }{ }^{42}\end{array}$ & $\begin{array}{l}12 \text { males that are } \\
\text { recreationally } \\
\text { active (mean age: } \\
23 \text { years old) with } \\
\text { no known } \\
\text { cardiovascular or } \\
\text { musculoskeletal } \\
\text { impairments or } \\
\text { prescribed } \\
\text { medications. }\end{array}$ & $\begin{array}{l}\text { Pneumatic cuff (10.5 } \\
\text { centimeters wide) } \\
\text { connected to an } \\
\text { automatic tourniquet } \\
\text { system, facemask } \\
\text { connected to an online } \\
\text { metabolic system, } \\
\text { manual } \\
\text { sphygmomanometer and } \\
\text { stethoscope. }\end{array}$ & $\begin{array}{l}\text { Unilateral elbow } \\
\text { flexion/extension with } \\
\text { dumbbells. There were } 4 \\
\text { different groups: heavy load } \\
\text { resistance exercise, light } \\
\text { load resistance exercise, } \\
\text { light load resistance } \\
\text { exercise with a continuous } \\
\text { low load pressure (20 } \\
\text { percent of } 1 \text { repetition } \\
\text { maximum) and light load } \\
\text { resistance exercise with an } \\
\text { intermittent high pressure. } \\
\text { Bicep curls for each group } \\
\text { were paced with a } \\
\text { metronome to ensure there } \\
\text { were } 2 \text { seconds for the } \\
\text { concentric phase and } 2 \\
\text { seconds for the eccentric. } \\
\text { Heavy load resistance was } \\
\text { performed at } 80 \text { percent of } 1 \\
\text { repetition maximum for } 4 \\
\text { sets of } 6 \text { to } 8 \text { repetitions. } \\
\text { Light load resistance, } \\
\text { constant BFR and } \\
\text { intermittent BFR exercises } \\
\text { are performed as } 4 \text { sets of } \\
\text { repetitions, the first is } 30 \\
\text { repetitions and the } \\
\text { following } 3 \text { are } 15 \\
\text { repetitions at } 20 \text { percent of } \\
\text { the patients' } 1 \text { repetition } \\
\text { maximum. }\end{array}$ & $\begin{array}{l}\text { Hemodynamic } \\
\text { measures (cardiac } \\
\text { output, stroke } \\
\text { volume, heart rate } \\
\text { and blood pressure } \\
\text { (both systolic and } \\
\text { diastolic) taken at } \\
\text { baseline, during } \\
\text { exercise, at } 5,20 \text {, } \\
40 \text { and } 60 \text { minutes } \\
\text { post-exercise. }\end{array}$ \\
\hline $\begin{array}{l}\text { Jessee et } \\
\text { al. }^{36}\end{array}$ & $\begin{array}{l}12 \text { male } \\
\text { participants aged } 18 \\
\text { to } 35 \text { years old that } \\
\text { were resistance- } \\
\text { trained. }\end{array}$ & $\begin{array}{l}\text { The cuff used was } 5 \\
\text { centimeters wide (model } \\
\text { SC5), chest press } \\
\text { machine and a doppler } \\
\text { probe (model MD6). }\end{array}$ & $\begin{array}{l}\text { Each participant was } \\
\text { subjected to } 4 \text { conditions: } \\
30 \text { percent of } 1 \text { repetition } \\
\text { maximum with differing } \\
\text { arterial occlusion pressures } \\
\text { ( } 0 \text { percent and } 40 \text { percent) }\end{array}$ & $\begin{array}{l}\text { Muscle thickness } \\
\text { change (chest and } \\
\text { triceps brachii) as } \\
\text { measured EMG } \\
\text { and ratings of } \\
\text { perceived exertion. }\end{array}$ \\
\hline
\end{tabular}


and 50 percent of 1

repetition maximum with

differing arterial occlusion

pressures $(0$ percent and 40

percent). Bench press was

the exercise implemented. 5

visits, separated by 5 to 10

days

Results of the study by Brandner et al. ${ }^{42}$ primarily evaluated the cardiovascular system.

The objective of the research was to determine which type of exercise limited hemodynamic stress. The high load resistive strengthening and intermittent BFR groups displayed the greatest rise in blood pressure, heart rate and cardiac output. Exercise effects on the cardiovascular system (with or without BFR) led authors to emphasize the importance of hemodynamic consideration before implementing new activities in compromised populations. Precautions taken with a vulnerable population are the reason that the researchers studied BFR in a healthy group of participants. The rationale for using a lower pressure with continuous BFR is for patient comfort considerations because the cuff remains on the extremity for a longer time period than with intermittent. ${ }^{42}$ However, the authors aimed to produce generalizable results that could provide more insight about BFR's indications in a non-active population, so that future protocols can be applied to unhealthy individuals without contraindications. ${ }^{42}$

Isolation of the upper extremity requires reducing many outside influences, which is difficult and may be the reason that upper extremity studies are minimally conducted. However, Brandner et al.'s ${ }^{42}$ study controlled for external influences by using a metronome for pacing, consistent arm positioning during exercise, and limiting compensatory movements by standing against a wall. Additionally, this study served as preliminary evidence that BFR does not produce negative long-term effects. This was demonstrated by the hemodynamic vital measures (blood pressure and heart rate) returning to baseline within as early as 5 minutes post exercise. ${ }^{42}$ 
In addition to cardiovascular effects, temporary musculoskeletal benefits were also observed in a study by Jessee et al. ${ }^{36}$ muscles of the chest increased in thickness after bench pressing. However, the increase was not statistically significant and only lasted 15 minutes. ${ }^{36}$

Use of BFR in Athletic Performance Training

Based on the previous information presented, BFR demonstrates many benefits in a variety of populations. Vopat et al. ${ }^{9}$ investigated further into the athletic population and examined 12 studies regarding athletic performance. Evidence from these studies, allowed Vopat et al. ${ }^{9}$ to conclude that low-load BFR training can supplement high-load workouts used in athletic practices or training in significantly enhancing muscle strength. Manimmanakorn et al. ${ }^{10}$ demonstrated that this increase in strength and muscle mass can translate into sport-specific, functional activities such as 5 meter sprint, 20 meter shuttle run and 505 agility. ${ }^{10}$ Yamanaka et al. ${ }^{49}$ conducted a study on Division I football players that were not naïve to resistance training and were required to have at least 5 years of experience. This study consisted of a control group (exercise without BFR) and an intervention group (exercise with BFR) and included both an upper and lower extremity exercise (bench press and squat, respectively). The study was implemented in the off-season and the bench press and squat were performed at 20 percent $1 \mathrm{RM}$ and 45 seconds rest between sets. BFR was implemented 3 times per week in addition to regular strength sessions for 4 weeks. Results of the study showed that there was a 7 percent increase in the 1RM for the bench press as well as an increase in upper and lower chest girth (by 3.7 centimeters and 2.6 centimeters, respectively). Additionally, there was an 8 percent increase in the $1 \mathrm{RM}$ for the squat, but no significant increase in thigh musculature girth. ${ }^{49}$ BFR use on athletes can be beneficial in a performance context. ${ }^{9,10}$ Safety Concerns 
Evaluating the efficacy of BFR requires determining if the benefits outweigh the risks. General risks of BFR were noted by the exclusion criteria of various studies. Vulnerable populations (such as patients with cardiovascular conditions) should not engage in BFR exercise without physician clearance and supervision. ${ }^{50}$ Although there is not much research on vulnerable populations, it is unethical to conduct research on them because of the increased risk for developing health problems.

Some examples of exclusion criteria in the study by Jessee et al. ${ }^{36}$ include history of thromboembolism or risk of thromboembolism (i.e. body mass index greater than or equal to 30), Crohn's disease, previous history of pelvis or femur fracture, varicose, major surgery or regular use of tobacco within the last 6 months. Giles et al. ${ }^{6}$ had similar criteria in terms of conditions related to thromboembolism, but elaborate further and included high blood pressure (characterized by a blood pressure greater than 140/90), diabetes, heart conditions and related symptoms (i.e. unexplained chest pain, fainting or dizzy spells during exercise) and pregnancy.

Thromboembolism, deep vein thrombosis or blood clot are all risks because BFR is interfering with blood flow. If the blood is stagnant in the extremity during the application of BFR, then individuals might have a higher likelihood of circulatory emergencies occurring. Conditions associated with unhealthy lifestyles such as obesity (associated with high BMI) and hypertension as well as tobacco use contribute to negative cardiovascular effects, which could explain why they are excluded.

Various fractures such as that of the femur or pelvis are of particular concern because of the vast blood supply contained within these bones. Additionally, there are blood networks surrounding these bones which could become disrupted in the event of a displaced or open 
fracture. Excessive internal bleeding can contribute to cardiovascular conditions, so individuals that have recently experienced any of these fracture types may be at higher risk.

For healthy populations there are some associated risks with BFR as well. Cook et al. ${ }^{40}$ observed symptoms of delayed onset muscle soreness (DOMS) and higher perceived exertion than in traditional resistance training. ${ }^{37}$ The presence of DOMS resulting from BFR is controversial among the research. Skin bruising may also occur but is a relatively benign adverse effect. ${ }^{3}$ Additionally, many studies do not show results of long-term effects, and there could be risks that researchers are not aware of yet. ${ }^{37}$

A particular risk that Hughes et al. ${ }^{38}$ and Tabata et al. ${ }^{37}$ studied is rhabdomyolysis. ${ }^{37,38}$ Rhabdomyolysis is a condition that involves a rapid breakdown of muscle that was injured, which can lead to kidney failure and become life-threatening. ${ }^{38}$ There are only two reported cases of rhabdomyolysis occurring in association with patients who exercised with BFR applied. The incidence of rhabdomyolysis occurring with BFR implementation is 0.008 percent. ${ }^{38}$ In one case, the patient was an obese, sedentary male, but had unremarkable family and personal history of muscle disorders such as rhabdomyolysis. He was 30 years old and, in an effort to improve his fitness level, he joined a gym. ${ }^{37,38}$

Context surrounding the development of rhabdomyolysis is important. Since it has such a low rate of occurrence, it is extremely unlikely, but with untrained supervision or lack of supervision and pre-existing illness or other health conditions, it is possible. On the patient's first day of working out, he performed exercises using BFR (with supervision of an individual that may or may not have been trained in BFR or strength coaching) and subsequently experienced extreme throat pain as well as pain in his arms and legs. This finding does not fit with the theory regarding BFR-induced rhabdomyolysis because the patient only used BFR to do leg exercises, 
not arm exercises, although pain was experienced in both. ${ }^{37}$ Additionally, the throat pain was attributed to a tonsillitis diagnosis that was detected by a white blood cell test. The tonsillitis was a confounding condition in this case that made it difficult to differentiate between symptoms of tonsillitis and rhabdomyolysis. Nevertheless, rhabdomyolysis was confirmed by blood tests and researchers of the case study concluded that the application of BFR on an individual with low baseline fitness was inappropriate. ${ }^{37}$ The credentials of the individual at the gym that assisted the patient in his training were never listed, so the trust value of the implemented training is low. If it were found that this individual is in fact, untrained in BFR strengthening, this could have contributed to the development of rhabdomyolysis. The "trainer's" use of BFR on this patient lead to full arterial occlusion, which is not a goal of BFR as it results in not only muscle ischemia (which is desired), but can also lead to muscle necrosis or death. ${ }^{37}$ Doctors managed the patient's condition in the hospital, following the prescription of 6 different medications used to treat the common cold/congestion, the flu, inflammation, bacterial infection, hemophilia (clotting disorder), and allergies. ${ }^{37}$ It is noted that the patient was treated in the hospital over a 10-day period and then recovered fully. ${ }^{37}$

Baseball, Fatigue and Recovery

There are over 50 games in the Division-I baseball regular season alone. ${ }^{33}$ The volume of games expected from pitchers requires them not only to be powerful with each pitch, but also have the endurance to pitch multiple innings and maintain longevity throughout the season. ${ }^{13}$ There are many factors involved in fatigue, but in pitching, fatigue can be objectively observed by decreased throwing velocity, decreased accuracy of pitches, increased arm soreness and an increased risk of injury. ${ }^{12}$ These can be influenced by both central nervous system (CNS) fatigue as well as peripheral nervous system fatigue (PNS). ${ }^{24} \mathrm{PNS}$ fatigue is associated with more local 
fatigue, typically to metabolic changes in particular extremity that is exerting force. On the other hand, CNS fatigue is more general, but also can cause fatigue to have a nociceptive (painful stimulus) perception from afferent fibers at a localized extremity ${ }^{24}$ Fatigue can significantly impact the neuromuscular pathway by impairing motor unit recruitment, neural drive and excitability. Essentially, fatigue that occurs with physical exertion alters neurological function. The efficiency of the nervous system is decreased with exercise-related fatigue, which lessens muscle force and causes physical performance to decline. ${ }^{51}$

Certain pitch types make a pitcher more susceptible to injury, including a breaking ball or a curve ball. ${ }^{12}$ Additionally, pitches associated with high pitch velocity (i.e. fastballs) can also contribute to higher incidences of injury. ${ }^{26}$ Understanding fatigue mechanisms affecting pitcher biomechanics is important to help combat fatigue. ${ }^{12}$ Reducing pitcher's fatigue with a fast recovery time can improve performance and decrease chance of injury. ${ }^{13}$ In a study by Whiteside et al., ${ }^{23}$ the proportion of fastballs thrown in the first inning was higher than those thrown towards the end of the game in the seventh inning, likely due to pitchers' fatigue from high velocity balls over the first six innings. This study demonstrated that pitchers compensated by throwing different pitches (i.e. breaking pitches) that required less speed. ${ }^{23}$ Livingston et al. ${ }^{12}$ found that isometric strength during shoulder rotation (both internal and external) was significantly reduced up to 2 days post-pitching bout. Researchers concluded that, to return to baseline strength, at least 3 days of rest are required before a pitcher returns. ${ }^{12}$ This becomes problematic, specifically for collegiate baseball players because schedules require play up to four games per week, which does not allow adequate recovery time. ${ }^{33}$ While there are multiple starting pitchers as well as bullpen pitchers, there is still such a high volume of games that it could increase pitchers' susceptibility to injury. ${ }^{13}$ Bullpen pitchers are also referred to as relief 
pitchers, which are brought in for strategic purposes or when the starting pitcher can no longer perform. $^{13}$

From a sensorimotor standpoint, baseball pitching heavily relies on glenohumeral, scapulothoracic and humeroulnar joint involvement. Each of these joints recover at different rates, with the glenohumeral requiring greater than 10 minutes to recover, 7 minutes for scapulothoracic and 4 minutes for humeroulnar. ${ }^{14}$ It should be noted that different pitch types require different patterns of muscle activation and place disbursed stresses on different joints. For example, curveballs place greater stress on the glenohumeral joint as opposed to the elbow. ${ }^{52}$ Curveballs increase the risk of shoulder pain by 52 percent, while the slider increases the risk of elbow pain by 86 percent. ${ }^{52}$ Other strategies to reduce injury rates among pitchers include implementing pitch count restrictions, ${ }^{10}$ developing pitching skills (i.e. increasing repertoire of different pitch styles to direct forces at different joints), ${ }^{23}$ reducing the number of innings pitched, ${ }^{12}$ avoiding the catcher position ${ }^{12}$ and decreasing the number of months pitched per year. $^{12}$

Figure B1. Fatigue, performance and injury rates linked. ${ }^{26}$

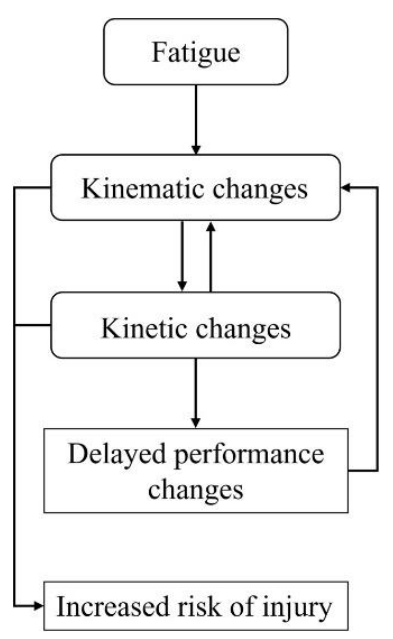

Figure B1 demonstrates the link between fatigue and performance. Baseball has been increasing in popularity significantly as of 2011 and addressing injury risks is essential to combat 
high injury rates. Microtrauma is commonly observed in baseball due to loads that accumulate over time due to high volumes of pitching. Unfortunately, due to the culture of baseball, many players are encouraged to continue pitching despite experiencing arm pain. Makhni et al. ${ }^{53}$ reported that almost half (46 percent) of youth pitchers continue throwing with arm pain and an even larger percentage ( 82 percent) complain of fatigue during both practices and games. To further demonstrate the link between fatigue and performance, Freeston et al. ${ }^{25}$ demonstrated that throwing velocity decreasing is a sign of general fatigue, whereas pitch accuracy and arm soreness are signs of fatigue specific to the arm. An increase in pitching volume also increases the need for BFR. ${ }^{15}$

Promoting recovery can also assist in retarding the progress of fatigue. "Recovery" has varying definitions among the literature, but in the context of baseball pitching, recovery is always discussed in relation to fatigue. Any strategies, tools, modalities or other resources that are used to diminish the negative physiological effects of fatigue and return body systems to baseline status are deemed recovery agents. ${ }^{24}$ The concept of recovery, demonstrates that fatigue can be diminished in as few as 4 to 7 minutes when using the appropriate methods. ${ }^{14}$ Some methods that were traditionally used for baseball recovery include active recovery (i.e. jogging to gradually reduce heart rate and restore blood flow to extremities), passive recovery (avoidance of physical activity for at least six minutes), or electrical stimulation (which delivers a low electrical current to the muscles exerted). ${ }^{13}$

Initially, it was thought that rest was the key to combat injury potential. Baseball programs began implementing the 100-pitch count limit to relieve pitchers and reduce overloading, however, injury rates continued to increase $\cdot{ }^{13}$ Furthermore, rest (also known as "passive recovery") in short intervals (likely between innings) causes the heart rate to decrease 
but does not promote effective blood flow, which is one of the primary goals of recovery. ${ }^{13}$ Other methods used in passive recovery between innings includes tasks such as wearing a jacket, towel or something else that can be used to insulate the pitching arm. ${ }^{54}$ The idea behind this is to keep the throwing arm loose in the dugout, but lacks scientific evidence and is founded in anecdotal success as well as psychological effects. ${ }^{54}$ Between-innings recovery is commonly seen among baseball programs using methods such as active rest. ${ }^{13}$ Active rest in its simplest form can be achieved by performing an exercise (such as jogging) at approximately 60 percent of maximum intensity (outlined as 60 percent of maximum heart rate). This gradually decreases the heart rate and optimizes blood flow to extremities which need replenishing. ${ }^{13}$

Modalities can also be useful in expediting the recovery process. While there an infinite number of recovery methods, commonly used modalities include electromuscular stimulation, pneumatic compression (Normatec) and cryotherapy application. Electromuscular stimulation has varying evidence among literature, but research by Warren et al. ${ }^{13}$ supports the idea that localized muscle contractions occurring contributes to increased oxygen, enabling the body to flush out the blood lactate that accumulates during physical exertion.

Pneumatic compression devices such as Normatec recovery boots target muscle glycogen restoration to promote recovery. ${ }^{31}$ Normatec devices have increased in popularity among the active community as a means for recovery. Normatec provides intermittent compression to the extremities via compartmentalized boots to promote oxygenation and blood flow following exercise. ${ }^{31}$ While some studies show that there is no benefit to using a pneumatic compressive device for recovery (as measured by muscle glycogen, blood lactate, plasma glucose and heart rate), there may be a psychological benefit to athletes that use Normatec as a "feel-good" modality. ${ }^{31}$ 
Cryotherapy is another tool that has demonstrated benefits in recovery, specifically in baseball pitchers. ${ }^{54}$ Intermittent cooling following pitching can combat overuse symptoms such as pain and swelling, as well as other anti-inflammatory effects. Additionally, intermittent cold application on the shoulder has demonstrated an increase in muscle fiber activation as well as the ability to assist with performance of repetitive motions. ${ }^{54}$ In a study by Bishop et al. ${ }^{54}$ wrapping an ice bag on the deltoid and forearm for six minutes resulted in higher pitch velocities later in a stimulated baseball game than without ice application between innings. Other studies investigated the effects of cryotherapy as well, but with modifications including: no pitcher's mound, combination treatment of cryotherapy and light exercise, and a single maximal pitch. However, due to much variability among cryotherapy applications, some literature has found that local cooling can contribute to delayed onset muscle soreness, which can contribute to faster fatigue and negatively affect performance. ${ }^{54}$

The use of BFR in recovery can be groundbreaking due to the physiological mechanisms behind cellular swelling. Restricting blood flow has the ability to completely change fluid dynamics in cells which stimulates protein synthesis, a key part of recovery. ${ }^{35}$ Replenishing and producing protein essential to tissue healing acutely after being subject to microtrauma that occurs during exercise will be beneficial in the long term. In a study that involved measuring fluid shift with an intervention that involved BFR without concomitant exercise, researchers found a decrease in plasma volume of 15 percent, which explained that cell swelling on the muscular level was due to a plasma fluid shift into the muscle. ${ }^{35}$ This demonstrated that inflating the cuff alone can increase protein synthesis, which can help reduce the microtrauma that is inherent to pitching. ${ }^{35}$

BFR Without Concomitant Exercise 
BFR has been described as a rehabilitation tool but recently made its debut as a recovery tool as well. One could infer that application of BFR as a recovery tool, rather than a rehabilitative tool would demonstrate a similar effect to Normatec, as their compressive properties mirror one another. However, Normatec is applied along the length of the extremity, while BFR is applied only at the proximal part, but displays metabolic changes in the entire extremity. ${ }^{31}$

In a systematic review by Cerqueira et al. ${ }^{32}$ three studies were included, all of which implemented BFR without concomitant exercise. In traditional BFR rehabilitation protocols, the cuff is placed on the proximal portion of an extremity and then goes through a circuit of exercises with reduced oxygen flowing to the extremity. However, without concomitant exercise, the cuff is simply inflated while the individual is at rest; a completely passive treatment for the patient. ${ }^{32}$ However, this type of protocol has only been investigated in a post-operative setting as opposed to a performance context.

The goal of the studies included in the systematic review by Cerqueira et al. ${ }^{32}$ was to decrease the amount of post-operative atrophy and strength deficits encountered by patients that were immobilized following an operating procedure. Two studies were conducted with healthy subjects while the third was on patients that underwent an ACL reconstruction. All three of the studies involved protocols with the lower extremity, where the cuff was placed on the proximal thigh and inflated for five minutes, followed by three minutes with the cuff deflated. This cycle alternating between inflation and deflation was repeated five times. ${ }^{32}$ The results of the studies have a risk of bias, but they demonstrated revolutionary results. When compared to their control counterparts (either no intervention or BFR cuff application with no inflation), the degree to which knee extensor strength was attenuated was significantly greater than the control, as 
measured by isokinetic and isometric torque. Additionally, girth measurements and crosssectional area (measured by MRI) of the thigh muscles demonstrated that BFR application maintained thigh circumference and contained the degree of disuse atrophy of the knee extensors. ${ }^{32}$ Since this study was limited to the lower extremity and a non-athletic population, further research needs to be conducted to determine if the benefits of BFR without exercise are more widely applicable.

Summary

BFR training is a concept in which occluding blood flow changes the intramuscular environment so that it takes on anaerobic properties. The abundance of metabolites and hormones promote muscle mass development and strength gains as well as increased neuromuscular function. BFR redefines the traditional use of strength training in a rehabilitative setting. Classically, strength training is done resistively at high intensities or volumes, whereas BFR is performed at a much lower load (a 30 to 60 percent difference in load according to 1RM). ${ }^{1-3,6,36,38,41}$ BFR can be applied in different patient populations and different extremities. BFR demonstrated strength and peak torque changes in the upper extremity and lower extremity in exercises such as squats/leg press, knee extension, calf raises, bicep curls and bench press. ${ }^{2-}$ ${ }^{4,6,48} \mathrm{BFR}$ use in a sport performance context has shown some improvement in functional activities such as sprinting and agility exercises. ${ }^{9}$ Historically, baseball pitchers used varying methods of techniques to reduce fatigue and promote recovery, in turn improving performance. ${ }^{12,26} \mathrm{BFR}$ without concomitant exercise has demonstrated benefits in terms of strength and attenuated atrophy in the lower extremity, but requires future studies for the upper extremity. ${ }^{32}$ However, there is limited data on baseball performance being influenced by BFR, 
but based on the physiological mechanisms described, one could infer that low-load BFR would have a positive effect in conjunction with sports training on pitching velocity of fastballs. 


\section{APPENDIX C}

\section{ADDITIONAL METHODS}

\section{$\underline{\text { Table C1. Fastball Pitch Count, Velocity Chart, Descriptive Statistics. }}$}

Game \#:

Subject \#:

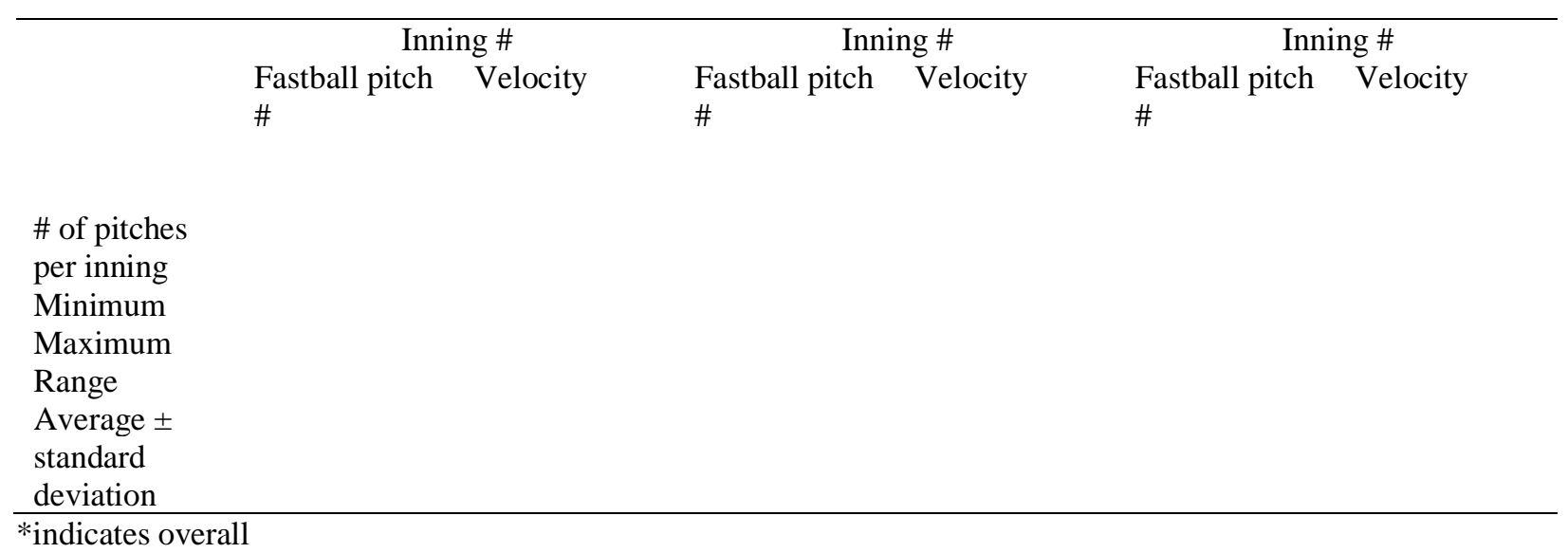


APPENDIX D

ADDITIONAL RESULTS

Table D1. Fastball Pitch Count, Velocity Chart, Descriptive Statistics.

Game \#: 1

Subject \#: 4

\begin{tabular}{|c|c|c|c|c|c|c|c|c|}
\hline & \multicolumn{2}{|c|}{ Inning 1} & \multicolumn{2}{|c|}{ Inning 2} & \multicolumn{2}{|c|}{ Inning 3} & \multicolumn{2}{|c|}{ Inning 4} \\
\hline & $\begin{array}{l}\text { Fastball } \\
\text { pitch \# }\end{array}$ & Velocity & $\begin{array}{l}\text { Fastball } \\
\text { pitch \# }\end{array}$ & Velocity & $\begin{array}{l}\text { Fastball } \\
\text { pitch \# }\end{array}$ & Velocity & $\begin{array}{l}\text { Fastball } \\
\text { pitch \# }\end{array}$ & Velocity \\
\hline & 1 & 86.8 & 13 & 87.7 & 25 & 88.2 & 42 & 88.5 \\
\hline & 2 & 87.1 & 14 & 88.9 & 26 & 87.8 & 43 & 87.6 \\
\hline & 3 & 88.4 & 15 & 88.8 & 27 & 89.1 & 44 & 89.7 \\
\hline & 4 & 87.9 & 16 & 88.7 & 28 & 88.3 & 45 & 88.1 \\
\hline & 5 & 88.2 & 17 & 88.3 & 29 & 88.3 & 46 & 88.8 \\
\hline & 6 & 87.8 & 18 & 87.9 & 30 & 89.6 & 47 & 86.7 \\
\hline & 7 & 89.1 & 19 & 87.0 & 31 & 87.1 & 48 & 88.8 \\
\hline & 8 & 88.2 & 20 & 88.0 & 32 & 87.4 & 49 & 88.7 \\
\hline & 9 & 87.9 & 21 & 86.7 & 33 & 87.6 & 50 & 87.5 \\
\hline & 10 & 88.2 & 22 & 88.5 & 34 & 87.4 & 51 & 87.7 \\
\hline & 11 & 88.3 & 23 & 87.8 & 35 & 89.4 & 52 & 87.1 \\
\hline & 12 & 89.4 & 24 & 87.5 & 36 & 88.6 & 53 & 86.8 \\
\hline & & & & & 37 & 88.6 & 54 & 88.5 \\
\hline & & & & & 38 & 88.7 & 55 & 88.0 \\
\hline & & & & & 39 & 88.5 & 56 & 86.2 \\
\hline & & & & & 40 & 89.0 & & \\
\hline & & & & & 41 & 88.3 & & \\
\hline $\begin{array}{l}\text { \# of } \\
\text { pitches } \\
\text { per inning }\end{array}$ & 12 & & 12 & & 17 & & 15 & \\
\hline Minimum & & 86.8 & & 86.7 & & 87.1 & & $86.2 *$ \\
\hline Maximum & & 89.4 & & 88.9 & & 89.6 & & $89.7 *$ \\
\hline Range & & 2.6 & & 2.2 & & 2.5 & & 3.5 \\
\hline $\begin{array}{l}\text { Average } \\
\pm \text { standard } \\
\text { deviation }\end{array}$ & & $\begin{array}{l}88.1 \pm \\
0.6\end{array}$ & & $88.0 \pm 0.7$ & & $88.3 \pm 0.7$ & & $87.9 \pm 1.0$ \\
\hline
\end{tabular}

\section{$\underline{\text { Table D2. Fastball Pitch Count, Velocity Chart, Descriptive Statistics. }}$}

Game \#: 2

Subject \#: 4

\begin{tabular}{|c|c|c|c|c|c|}
\hline \multicolumn{2}{|c|}{ Inning 1} & \multicolumn{2}{|c|}{ Inning 2} & \multicolumn{2}{|c|}{ Inning 3} \\
\hline $\begin{array}{l}\text { Fastball pitch } \\
\#\end{array}$ & Velocity & $\begin{array}{l}\text { Fastball pitch } \\
\#\end{array}$ & Velocity & $\begin{array}{l}\text { Fastball pitch } \\
\#\end{array}$ & Velocity \\
\hline 1 & 87.6 & 12 & 88.6 & 26 & 88.4 \\
\hline 2 & 89.5 & 13 & 89.3 & 27 & 88.6 \\
\hline 3 & 90.2 & 14 & 88.7 & 28 & 88.3 \\
\hline 4 & 88.6 & 15 & 89.7 & 29 & 89.3 \\
\hline 5 & 89.5 & 16 & 89.6 & 30 & 89.5 \\
\hline 6 & 90.3 & 17 & 90.3 & 31 & 89.5 \\
\hline
\end{tabular}




\begin{tabular}{|c|c|c|c|c|c|c|}
\hline & 7 & 89.2 & 18 & 89.8 & 32 & 89.7 \\
\hline & 8 & 89.9 & 19 & 90.3 & 33 & 89.9 \\
\hline & 9 & 88.9 & 20 & 91.3 & 34 & 88.9 \\
\hline & 10 & 89.8 & 21 & 89.5 & 35 & 90.1 \\
\hline & 11 & 89.5 & 22 & 91.1 & 36 & 89.6 \\
\hline & & & 23 & 90.4 & & \\
\hline & & & 24 & 89.6 & & \\
\hline & & & 25 & 89.8 & & \\
\hline $\begin{array}{l}\text { \# of pitches } \\
\text { per inning }\end{array}$ & 11 & & 14 & & 11 & \\
\hline Minimum & & $87.6^{*}$ & & 88.6 & & 88.3 \\
\hline Maximum & & $90.3^{*}$ & & 91.3 & & 90.1 \\
\hline Range & & 2.7 & & 2.7 & & 1.8 \\
\hline $\begin{array}{l}\text { Average } \\
\pm \text { standard } \\
\text { deviation }\end{array}$ & & $89.4 \pm 0.8$ & & $89.8 \pm 0.8$ & & $89.3 \pm 0.6$ \\
\hline
\end{tabular}

Table D3. Fastball Pitch Count, Velocity Chart, Descriptive Statistics.

Game \#: 3

Subject \#: 1

\begin{tabular}{|c|c|c|c|c|c|c|c|c|}
\hline & \multicolumn{2}{|c|}{ Inning 1} & \multicolumn{2}{|c|}{ Inning 2} & \multicolumn{2}{|c|}{ Inning 3} & \multicolumn{2}{|c|}{ Inning 4} \\
\hline & $\begin{array}{l}\text { Fastball } \\
\text { pitch \# }\end{array}$ & Velocity & $\begin{array}{l}\text { Fastball } \\
\text { pitch \# }\end{array}$ & Velocity & $\begin{array}{l}\text { Fastball } \\
\text { pitch \# }\end{array}$ & Velocity & $\begin{array}{l}\text { Fastball } \\
\text { pitch \# }\end{array}$ & Velocity \\
\hline & 1 & 92.1 & 8 & 93.1 & 17 & 92.4 & 22 & 94.1 \\
\hline & 2 & 93.4 & 9 & 94.2 & 18 & 95.2 & 23 & 96.1 \\
\hline & 3 & 93.8 & 10 & 94.0 & 19 & 93.8 & 24 & 96.4 \\
\hline & 4 & 93.0 & 11 & 93.5 & 20 & 96.1 & 25 & 94.6 \\
\hline & 5 & 95.2 & 12 & 95.5 & 21 & 93.8 & 26 & 93.9 \\
\hline & 6 & 94.0 & 13 & 94.2 & & & & \\
\hline & 7 & 94.4 & 14 & 95.2 & & & & \\
\hline & & & 15 & 93.5 & & & & \\
\hline & & & 16 & 94.7 & & & & \\
\hline $\begin{array}{l}\text { \# of } \\
\text { pitches } \\
\text { per inning }\end{array}$ & 7 & & 9 & & 5 & & 5 & \\
\hline Minimum & & 92.1 & & 93.1 & & 92.4 & & 93.9 \\
\hline Maximum & & 95.2 & & 95.5 & & 96.1 & & $96.4 *$ \\
\hline Range & & 3.1 & & 2.4 & & 3.7 & & 2.5 \\
\hline $\begin{array}{l}\text { Average } \pm \\
\text { standard } \\
\text { deviation }\end{array}$ & & $93.7 \pm 1.0$ & & $94.2 \pm 0.8$ & & $94.3 \pm 1.4$ & & $95.0 \pm 1.2$ \\
\hline
\end{tabular}

Table D4. Fastball Pitch Count, Velocity Chart, Descriptive Statistics Continued.

Game \#: 3

Subject \#: 1

\begin{tabular}{|c|c|c|c|}
\hline Inning 5 & Inning 6 & Inning 7 & Inning 8 \\
\hline $\begin{array}{l}\text { Fastball } \\
\text { pitch \# }\end{array}$ & $\begin{array}{l}\text { Fastball } \\
\text { pitch \# }\end{array}$ & $\begin{array}{l}\text { Fastball } \\
\text { pitch \# }\end{array}$ & $\begin{array}{l}\text { Fastball } \\
\text { pitch \# }\end{array}$ \\
\hline
\end{tabular}




\begin{tabular}{|c|c|c|c|c|c|c|c|c|}
\hline & 27 & 86.5 & 34 & 94.1 & 39 & 93.3 & 46 & 93.9 \\
\hline & 28 & 96.0 & 35 & 95.8 & 40 & 94.2 & 47 & 94.0 \\
\hline & 29 & 95.2 & 36 & 95.8 & 41 & 94.4 & 48 & 93.7 \\
\hline & 30 & 95.5 & 37 & 95.4 & 42 & 94.2 & 49 & 92.4 \\
\hline & 31 & 95.9 & 38 & 95.0 & 43 & 95.4 & 50 & 92.1 \\
\hline & 32 & 95.5 & & & 44 & 94.4 & 51 & 92.8 \\
\hline & 33 & 95.7 & & & 45 & 88.2 & 52 & 94.4 \\
\hline $\begin{array}{l}\text { \# of } \\
\text { pitches } \\
\text { per inning }\end{array}$ & 7 & & 5 & & 7 & & 7 & \\
\hline Minimum & & $86.5^{*}$ & & 94.1 & & 88.2 & & 92.1 \\
\hline Maximum & & 96.0 & & 95.8 & & 95.4 & & 94.4 \\
\hline Range & & 9.5 & & 1.7 & & 7.2 & & 2.3 \\
\hline $\begin{array}{l}\text { Average } \\
\pm \text { standard } \\
\text { deviation }\end{array}$ & & $94.3 \pm 3.4$ & & $95.2 \pm 0.7$ & & $93.4 \pm 2.4$ & & $93.3 \pm 0.9$ \\
\hline
\end{tabular}

Table D5. Fastball Pitch Count, Velocity Chart, Descriptive Statistics.

Game \#: 4

Subject \#: 2

\begin{tabular}{|c|c|c|c|c|c|c|}
\hline & \multicolumn{2}{|c|}{ Inning 1} & \multicolumn{2}{|c|}{ Inning 2} & \multicolumn{2}{|c|}{ Inning 3} \\
\hline & $\begin{array}{l}\text { Fastball pitch } \\
\#\end{array}$ & Velocity & $\begin{array}{l}\text { Fastball pitch } \\
\#\end{array}$ & Velocity & $\begin{array}{l}\text { Fastball pitch } \\
\#\end{array}$ & Velocity \\
\hline & 1 & 87.2 & 9 & 86.3 & 21 & 85.8 \\
\hline & 2 & 86.9 & 10 & 86.8 & & \\
\hline & 3 & 86.9 & 11 & 85.5 & & \\
\hline & 4 & 87.1 & 12 & 86.4 & & \\
\hline & 5 & 86.9 & 13 & 86.9 & & \\
\hline & 6 & 86.4 & 14 & 86.7 & & \\
\hline & 7 & 87.9 & 15 & 86.4 & & \\
\hline & 8 & 87.0 & 16 & 82.9 & & \\
\hline & & & 17 & 87.4 & & \\
\hline & & & 18 & 86.7 & & \\
\hline & & & 19 & 87.1 & & \\
\hline & & & 20 & 87.2 & & \\
\hline $\begin{array}{l}\text { \# of pitches } \\
\text { per inning }\end{array}$ & 8 & & 12 & & 1 & \\
\hline Minimum & & 86.4 & & $82.9^{*}$ & & 85.8 \\
\hline Maximum & & $87.9 *$ & & 87.4 & & 85.8 \\
\hline Range & & 1.5 & & 4.5 & & 0.0 \\
\hline $\begin{array}{l}\text { Average } \\
\pm \text { standard } \\
\text { deviation }\end{array}$ & & $87.0 \pm 0.4$ & & $86.4 \pm 1.2$ & & $85.8 \pm 0.0$ \\
\hline
\end{tabular}

Table D6. Fastball Pitch Count, Velocity Chart, Descriptive Statistics.

Game \#: 5

Subject \#: 3 


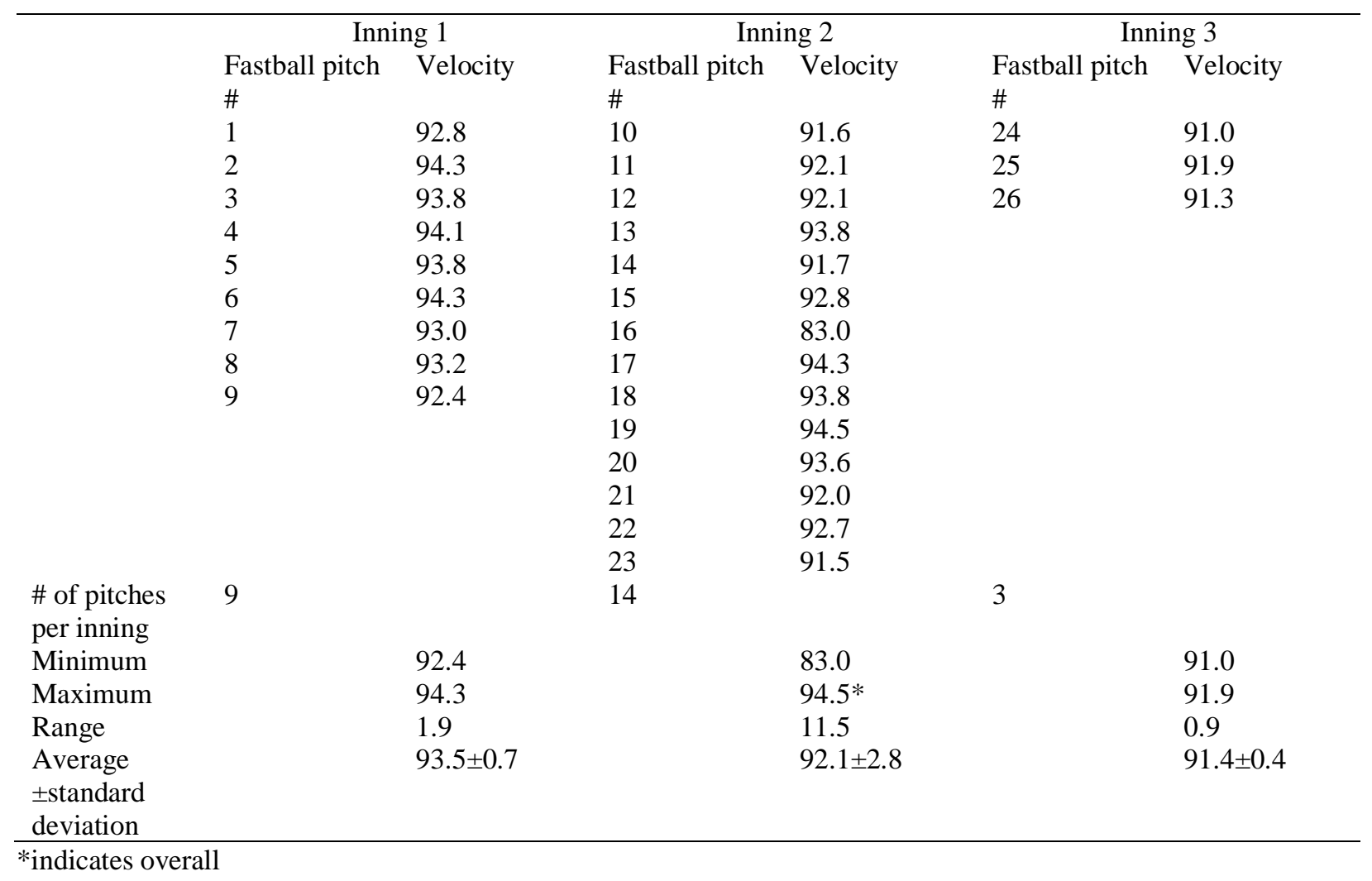

Table D7. Fastball Pitch Count, Velocity Chart, Descriptive Statistics Continued.

Game \#: 5

Subject \#: 3

\# of pitches 11

5

5

per inning

Minimum

91.0

91.9

92.3

91.2

91.6

92.1

92.3

92.2

91.4

91.5

91.0

36

91.8

Maximum

92.3

\begin{tabular}{llll}
\multicolumn{3}{c}{ Inning 5 } & \multicolumn{2}{c}{ Inning 6 } \\
Fastball pitch & Velocity & Fastball pitch & Velocity \\
$\#$ & & $\#$ & \\
38 & 88.5 & 43 & 82.9 \\
39 & 90.6 & 44 & 90.2 \\
40 & 92.0 & 45 & 90.9 \\
41 & 91.6 & 46 & 90.5 \\
42 & 91.3 & 47 & \\
& & &
\end{tabular}




\begin{tabular}{llll} 
Range & 1.3 & 3.5 & 8.4 \\
Average & $91.8 \pm 0.4$ & $90.8 \pm 1.4$ & $89.2 \pm 3.5$ \\
\pm standard & & & \\
deviation & & & \\
\hline *indicates overall & &
\end{tabular}

Table D8. Fastball Pitch Count, Velocity Chart, Descriptive Statistics.

Game \#: 6

Subject \#: 4

\begin{tabular}{|c|c|c|c|c|c|c|c|c|}
\hline & \multicolumn{2}{|c|}{ Inning 1} & \multicolumn{2}{|c|}{ Inning 2} & \multicolumn{2}{|c|}{ Inning 3} & \multicolumn{2}{|c|}{ Inning 4} \\
\hline & $\begin{array}{l}\text { Fastball } \\
\text { pitch \# }\end{array}$ & Velocity & $\begin{array}{l}\text { Fastball } \\
\text { pitch \# }\end{array}$ & Velocity & $\begin{array}{l}\text { Fastball } \\
\text { pitch \# }\end{array}$ & Velocity & $\begin{array}{l}\text { Fastball } \\
\text { pitch \# }\end{array}$ & Velocity \\
\hline & 1 & 84.8 & 11 & 86.1 & 25 & 85.3 & 44 & 87.0 \\
\hline & 2 & 86.0 & 12 & 87.4 & 26 & 85.4 & 45 & 88.0 \\
\hline & 3 & 86.7 & 13 & 87.9 & 27 & 86.9 & 46 & 87.9 \\
\hline & 4 & 86.9 & 14 & 88.1 & 28 & 87.9 & 47 & 88.5 \\
\hline & 5 & 87.3 & 15 & 87.4 & 29 & 88.4 & 48 & 87.6 \\
\hline & 6 & 86.4 & 16 & 86.7 & 30 & 86.9 & 49 & 89.6 \\
\hline & 7 & 87.0 & 17 & 87.3 & 31 & 88.0 & 50 & 88.5 \\
\hline & 8 & 88.3 & 18 & 87.8 & 32 & 88.2 & 51 & 88.9 \\
\hline & 9 & 87.2 & 19 & 87.0 & 33 & 89.4 & 52 & 89.4 \\
\hline & 10 & 88.3 & 20 & 87.7 & 34 & 88.6 & 53 & 85.8 \\
\hline & & & 21 & 86.9 & 35 & 88.3 & 54 & 87.7 \\
\hline & & & 22 & 89.2 & 36 & 88.5 & 55 & 87.2 \\
\hline & & & 23 & 87.8 & 37 & 88.6 & 56 & 88.3 \\
\hline & & & 24 & 88.2 & 38 & 88.1 & 57 & 89.3 \\
\hline & & & & & 39 & 88.2 & 58 & 88.6 \\
\hline & & & & & 40 & 87.8 & 59 & 87.4 \\
\hline & & & & & 41 & 88.6 & 60 & 88.7 \\
\hline & & & & & 42 & 88.5 & & \\
\hline & & & & & 43 & 88.1 & & \\
\hline $\begin{array}{l}\# \text { of } \\
\text { pitches } \\
\text { per inning }\end{array}$ & 19 & & 14 & & 19 & & 17 & \\
\hline Minimum & & $84.8^{*}$ & & 86.1 & & 85.3 & & 85.8 \\
\hline Maximum & & 88.3 & & 89.2 & & 89.4 & & $89.6 *$ \\
\hline Range & & 3.5 & & 3.1 & & 4.1 & & 4.3 \\
\hline $\begin{array}{l}\text { Average } \pm \\
\text { standard } \\
\text { deviation }\end{array}$ & & $86.9 \pm 1.0$ & & $87.5 \pm 0.7$ & & $87.9 \pm 1.0$ & & $88.1 \pm 1.0$ \\
\hline
\end{tabular}

\section{Table D9. Fastball Pitch Count, Velocity Chart, Descriptive Statistics.}

Game \#: 7

Subject \#: 1

\begin{tabular}{llllllll}
\multicolumn{2}{c}{ Inning 1 } & \multicolumn{2}{c}{ Inning 2 } & \multicolumn{2}{c}{ Inning 3 } & \multicolumn{2}{c}{ Inning 4 } \\
$\begin{array}{l}\text { Fastball } \\
\text { pitch \# }\end{array}$ & Velocity & $\begin{array}{l}\text { Fastball } \\
\text { pitch \# }\end{array}$ & Velocity & $\begin{array}{l}\text { Fastball } \\
\text { pitch \# }\end{array}$ & Velocity & Fastball & Velocity \\
1 & 92.4 & 12 & 92.8 & 21 & 90.6 & 27 & 91.8 \\
2 & 92.6 & 13 & 92.7 & 22 & 93.2 & 28 & 92.4
\end{tabular}




\begin{tabular}{|c|c|c|c|c|c|c|c|c|}
\hline & 3 & 93.1 & 14 & 93.6 & 23 & 92.3 & 29 & 92.5 \\
\hline & 4 & 94.9 & 15 & 93.5 & 24 & 93.8 & 30 & 92.7 \\
\hline & 5 & 94.7 & 16 & 93.8 & 25 & 94.2 & 31 & 92.5 \\
\hline & 6 & 94.5 & 17 & 93.3 & 26 & 92.5 & 32 & 93.0 \\
\hline & 7 & 92.8 & 18 & 93.7 & & & 33 & 93.4 \\
\hline & 8 & 93.7 & 19 & 92.9 & & & 34 & 95.5 \\
\hline & 9 & 92.8 & 20 & 93.1 & & & & \\
\hline & 10 & 94.4 & & & & & & \\
\hline & 11 & 93.2 & & & & & & \\
\hline $\begin{array}{l}\text { \# of } \\
\text { pitches } \\
\text { per inning }\end{array}$ & 11 & & 9 & & 6 & & 8 & \\
\hline Minimum & & 92.4 & & 92.7 & & $90.6^{*}$ & & 91.8 \\
\hline Maximum & & 94.9 & & 93.8 & & 94.2 & & $95.5^{*}$ \\
\hline Range & & 2.5 & & 1.1 & & 3.6 & & 3.7 \\
\hline $\begin{array}{l}\text { Average } \pm \\
\text { standard } \\
\text { deviation }\end{array}$ & & $93.6 \pm 0.9$ & & $93.3 \pm 0.4$ & & $92.8 \pm 1.3$ & & $93.0 \pm 1.1$ \\
\hline
\end{tabular}

Table D10. Fastball Pitch Count, Velocity Chart, Descriptive Statistics Continued.

Game \#: 7

Subject \#: 1

\begin{tabular}{|c|c|c|c|c|c|c|}
\hline & & $\lg 5$ & & $\operatorname{lng} 7$ & Inn & $\lg 8$ \\
\hline & $\begin{array}{l}\text { Fastball pitch } \\
\#\end{array}$ & Velocity & $\begin{array}{l}\text { Fastball pitch } \\
\#\end{array}$ & Velocity & $\begin{array}{l}\text { Fastball pitch } \\
\#\end{array}$ & Velocity \\
\hline & 35 & 92.3 & 39 & 92.9 & 50 & 91.6 \\
\hline & 36 & 94.7 & 40 & 93.3 & 51 & 93.6 \\
\hline & 37 & 94.0 & 41 & 91.3 & 52 & 93.7 \\
\hline & 38 & 93.9 & 42 & 93.1 & & \\
\hline & & & 43 & 93.5 & & \\
\hline & & & 44 & 92.6 & & \\
\hline & & & 45 & 93.1 & & \\
\hline & & & 46 & 93.6 & & \\
\hline & & & 47 & 93.6 & & \\
\hline & & & 48 & 94.1 & & \\
\hline & & & 49 & 94.6 & & \\
\hline $\begin{array}{l}\text { \# of pitches } \\
\text { per inning }\end{array}$ & 4 & & 11 & & 3 & \\
\hline Minimum & & 92.3 & & 91.4 & & 91.6 \\
\hline Maximum & & 94.7 & & 94.6 & & 93.7 \\
\hline Range & & 2.4 & & 3.2 & & 2.1 \\
\hline $\begin{array}{l}\text { Average } \\
\pm \text { standard } \\
\text { deviation }\end{array}$ & & $93.7 \pm 1.0$ & & $93.2 \pm 0.8$ & & $93.0 \pm 1.1$ \\
\hline
\end{tabular}

Table D11. Fastball Pitch Count, Velocity Chart, Descriptive Statistics.

Game \#: 8

Subject \#: 2 


\begin{tabular}{|c|c|c|c|c|c|c|}
\hline & \multicolumn{2}{|c|}{ Inning 1} & \multicolumn{2}{|c|}{ Inning 2} & \multicolumn{2}{|c|}{ Inning 3} \\
\hline & $\begin{array}{l}\text { Fastball pitch } \\
\#\end{array}$ & Velocity & $\begin{array}{l}\text { Fastball pitch } \\
\#\end{array}$ & Velocity & $\begin{array}{l}\text { Fastball pitch } \\
\#\end{array}$ & Velocity \\
\hline & 1 & 90.3 & 15 & 88.7 & 29 & 87.7 \\
\hline & 2 & 90.4 & 16 & 89.3 & 30 & 88.2 \\
\hline & 3 & 89.3 & 17 & 85.7 & 31 & 88.2 \\
\hline & 4 & 89.5 & 18 & 89.9 & 32 & 89.8 \\
\hline & 5 & 90.2 & 19 & 85.3 & 33 & 89.2 \\
\hline & 6 & 89.9 & 20 & 89.1 & 34 & 88.2 \\
\hline & 7 & 90.3 & 21 & 89.5 & 35 & 88.5 \\
\hline & 8 & 90.4 & 22 & 86.7 & 36 & 88.2 \\
\hline & 9 & 86.0 & 23 & 90.7 & 37 & 89.5 \\
\hline & 10 & 84.6 & 24 & 87.5 & & \\
\hline & 11 & 89.3 & 25 & 90.5 & & \\
\hline & 12 & 90.9 & 26 & 89.6 & & \\
\hline & 13 & 90.4 & 27 & 88.2 & & \\
\hline & 14 & 90.0 & 28 & 88.7 & & \\
\hline $\begin{array}{l}\text { \# of pitches } \\
\text { per inning }\end{array}$ & 14 & & 14 & & 9 & \\
\hline Minimum & & $84.6^{*}$ & & 85.3 & & 87.7 \\
\hline Maximum & & $90.9 *$ & & 90.7 & & 89.8 \\
\hline Range & & 6.3 & & 5.4 & & 2.1 \\
\hline $\begin{array}{l}\text { Average } \\
\pm \text { standard } \\
\text { deviation }\end{array}$ & & $89.4 \pm 1.8$ & & $88.5 \pm 1.7$ & & $88.6 \pm 0.7$ \\
\hline
\end{tabular}

Table D12. Fastball Pitch Count, Velocity Chart, Descriptive Statistics Continued.

Game \#: 8

Subject \#: 2

\begin{tabular}{lllll}
\hline & \multicolumn{2}{c}{ Inning 4} & \multicolumn{2}{c}{ Inning 5 } \\
& Fastball pitch \# & Velocity & Fastball pitch \# & Velocity \\
& 38 & 87.4 & 54 & 85.6 \\
& 39 & 87.2 & 55 & 87.3 \\
& 40 & 87.9 & 56 & 87.9 \\
& 41 & 88.6 & 57 & 88.4 \\
& 42 & 88.8 & 58 & 87.1 \\
& 43 & 87.8 & 59 & 87.2 \\
& 44 & 88.1 & 60 & 88.0 \\
& 45 & 89.8 & 61 & \\
& 46 & 87.4 & 62 & \\
& 47 & 88.0 & 63 & \\
\# of pitches per & 48 & 87.7 & & \\
inning & 49 & 87.8 & & \\
Minimum & 50 & 89.4 & & \\
Maximum & 51 & 88.1 & & \\
Range & 52 & 88.2 & & \\
& 16 & 88.1 & & \\
& & & & \\
& & 87.2 & & \\
& & 2.6 & & \\
\end{tabular}


Average \pm standard

$88.1 \pm 0.7$

$87.2 \pm 0.9$

deviation

*indicates overall

Table D13. Fastball Pitch Count, Velocity Chart, Descriptive Statistics.

Game \#: 9

Subject \#: 3

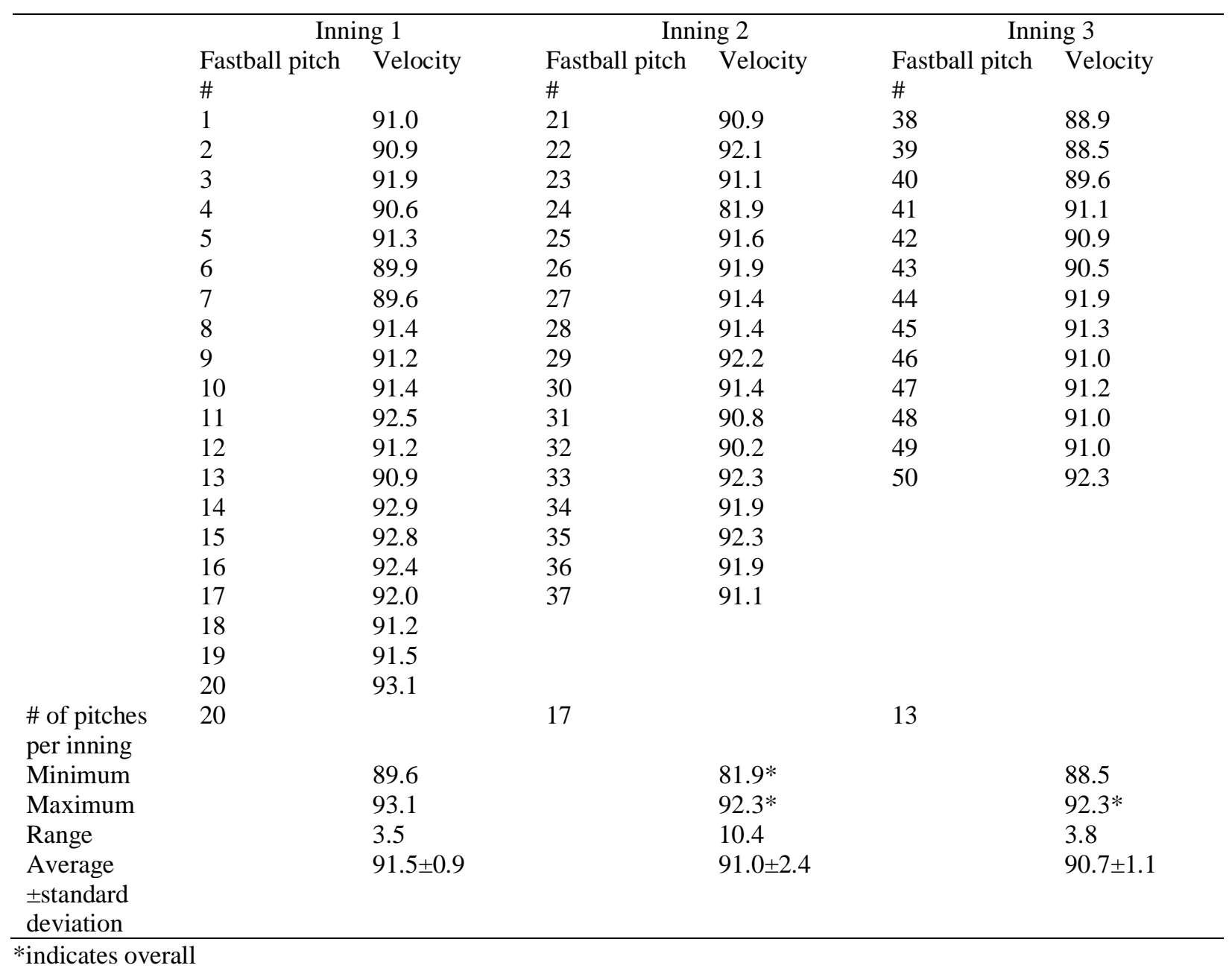

Table D14. Fastball Pitch Count, Velocity Chart, Descriptive Statistics Continued.

Game \#: 9

Subject \#: 3

\begin{tabular}{llll}
\multicolumn{2}{c}{ Inning 4 } & \multicolumn{2}{c}{ Inning 5 } \\
Fastball pitch \# & Velocity & Fastball pitch \# & Velocity \\
51 & 89.4 & 67 & 88.2 \\
52 & 89.3 & 68 & 89.3 \\
53 & 90.8 & 69 & 89.7 \\
54 & 92.0 & 70 & 89.5 \\
55 & 90.9 & 71 & 92.0
\end{tabular}




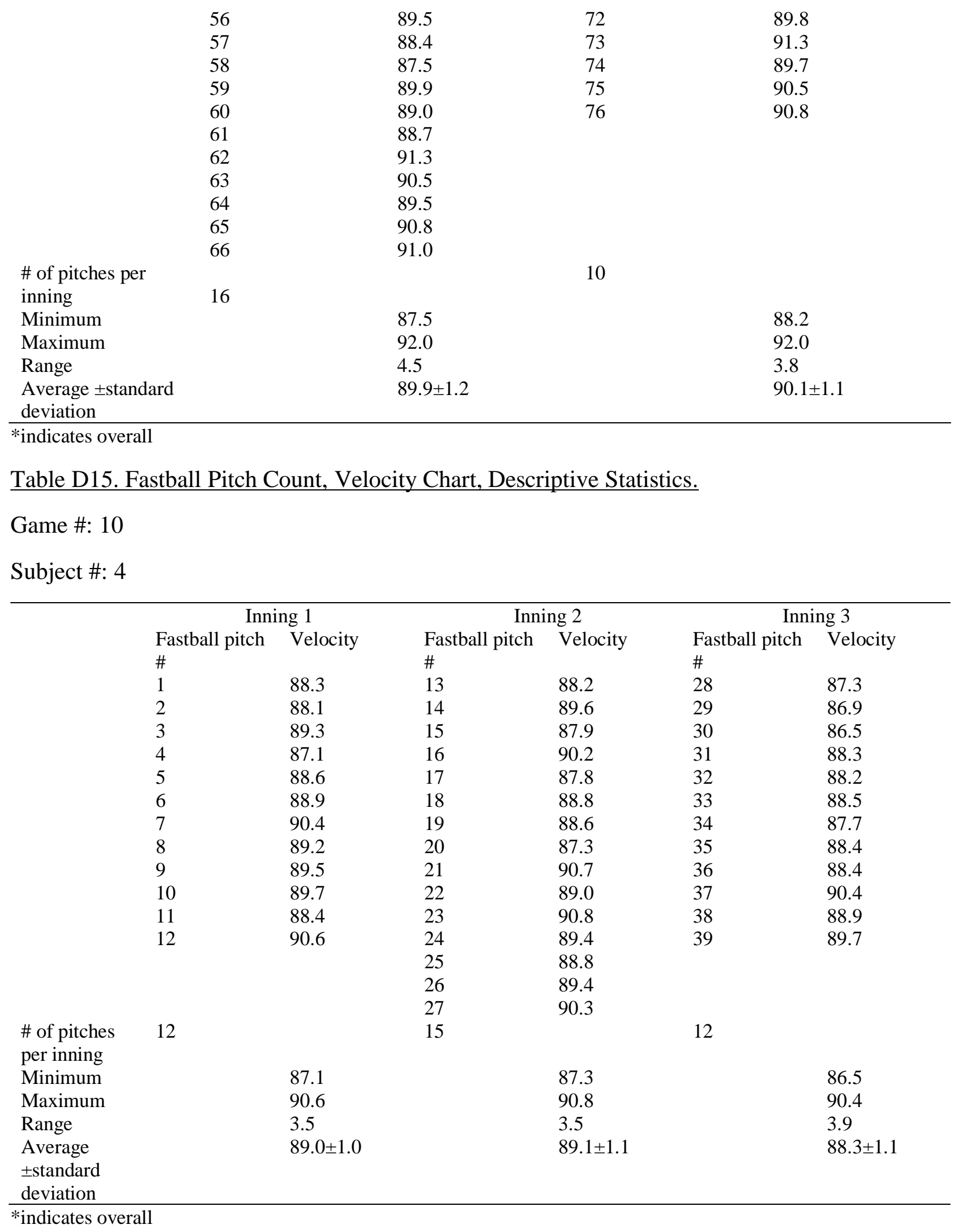

Table D16. Fastball Pitch Count, Velocity Chart, Descriptive Statistics Continued. 
Game \#: 10

Subject \#: 4

\begin{tabular}{|c|c|c|c|c|}
\hline & \multicolumn{2}{|c|}{ Inning 4} & \multicolumn{2}{|c|}{ Inning 5} \\
\hline & Fastball pitch \# & Velocity & Fastball pitch \# & Velocity \\
\hline & 40 & 89.7 & 52 & 89.7 \\
\hline & 41 & 90.5 & 53 & 89.0 \\
\hline & 42 & 90.3 & 54 & 89.3 \\
\hline & 43 & 89.7 & 55 & 89.8 \\
\hline & 44 & 91.7 & 56 & 86.8 \\
\hline & 45 & 91.4 & 57 & 87.9 \\
\hline & 46 & 90.6 & 58 & 90.9 \\
\hline & 47 & 90.6 & 59 & 89.1 \\
\hline & 48 & 81.1 & 60 & 89.5 \\
\hline & 49 & 87.6 & 61 & 90.4 \\
\hline & 50 & 88.1 & 62 & 86.3 \\
\hline & 51 & 87.7 & 63 & 87.2 \\
\hline & & & 64 & 87.9 \\
\hline & & & 65 & 88.3 \\
\hline & & & 66 & 88.8 \\
\hline \# of pitches per & & & 15 & \\
\hline inning & 12 & & & \\
\hline Minimum & & $81.1 *$ & & 86.3 \\
\hline Maximum & & $91.7 *$ & & 90.9 \\
\hline Range & & 10.6 & & 4.6 \\
\hline $\begin{array}{l}\text { Average } \pm \text { standard } \\
\text { deviation }\end{array}$ & & $89.1 \pm 2.9$ & & $88.7 \pm 1.3$ \\
\hline
\end{tabular}

\section{Table D17. Fastball Pitch Count, Velocity Chart, Descriptive Statistics.}

Game \#: 11

Subject \#: 1

\# of

pitches per 9

inning

Minimum

94.9

13

Inning $1 \quad$ Inning 2 Inning 3

Fastball Velocity Fastball Velocity

pitch \#

$194.9-10$

2

3

4

5

7
8 $96.9 \quad 16$

$8 \quad 97.5 \quad 17$

$9 \quad 96.6 \quad 18$

19

20

21

22

$95.7 \quad 23 \quad 95.0$

$\begin{array}{lll}95.1 & 24 & 95.8\end{array}$

$\begin{array}{lll}95.5 & 25 & 97.2\end{array}$

$96.1 \quad 26$

$95.4 \quad 27$

$96.1 \quad 28$

$95.6 \quad 29$

$95.8 \quad 30$

$96.1 \quad 31$

96.4

95.8

95.2

97.8

95.8

97.1

96.3

95.8

95.9

95.4
9

95.1
Inning 4 Fastball Velocity Fastball Velocity pitch \# pitch \#

$\begin{array}{lll}32 & 86.7 & 37\end{array}$

$\begin{array}{llll}33 & 95.4 & 38 & 95.1\end{array}$

$\begin{array}{llll}34 & 96.3 & 39 & 95.2\end{array}$

$\begin{array}{llll}35 & 96.4 & 40 & 96.1\end{array}$

$\begin{array}{llll}36 & 95.4 & 41 & 95.4\end{array}$

$42 \quad 96.2$

\section{(1)}




$\begin{array}{llllll}\text { Maximum } & 97.5 & 97.8^{*} & 97.2 & 96.4 & 96.2 \\ \text { Range } & 2.6 & 2.7 & 2.2 & 9.7 & 2.6 \\ \text { Average } \pm & 96.0 \pm 0.9 & 95.9 \pm 0.7 & 96.0 \pm 0.7 & 94.1 \pm 4.1 & 95.3 \pm 0.9 \\ \text { standard } & & & & & \\ \text { deviation } & & & & \end{array}$

Table D18. Fastball Pitch Count, Velocity Chart, Descriptive Statistics Continued.

Game \#: 11

Subject \#: 1

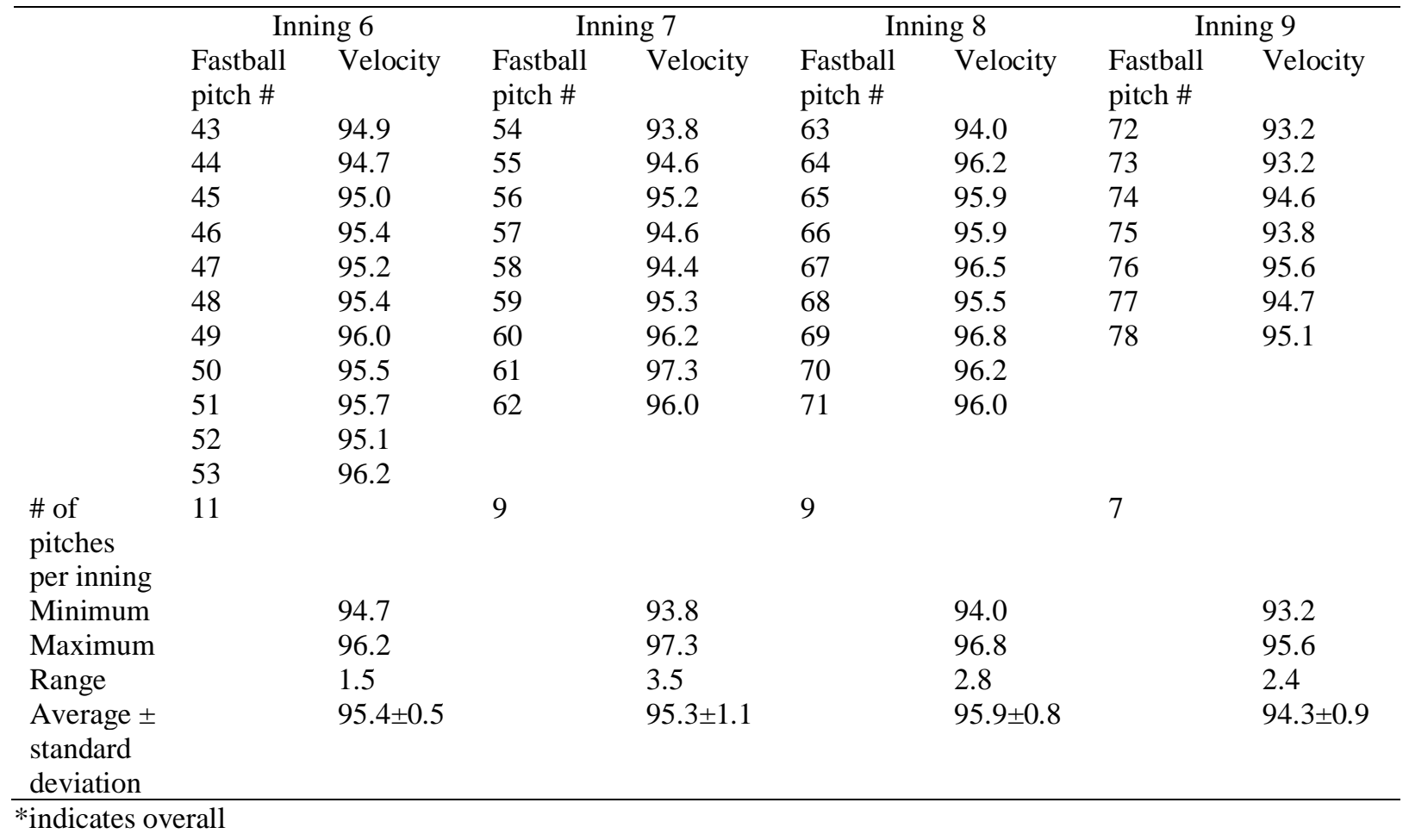

Table D19. Fastball Pitch Count, Velocity Chart, Descriptive Statistics.

Game \#: 12

Subject \#: 2 


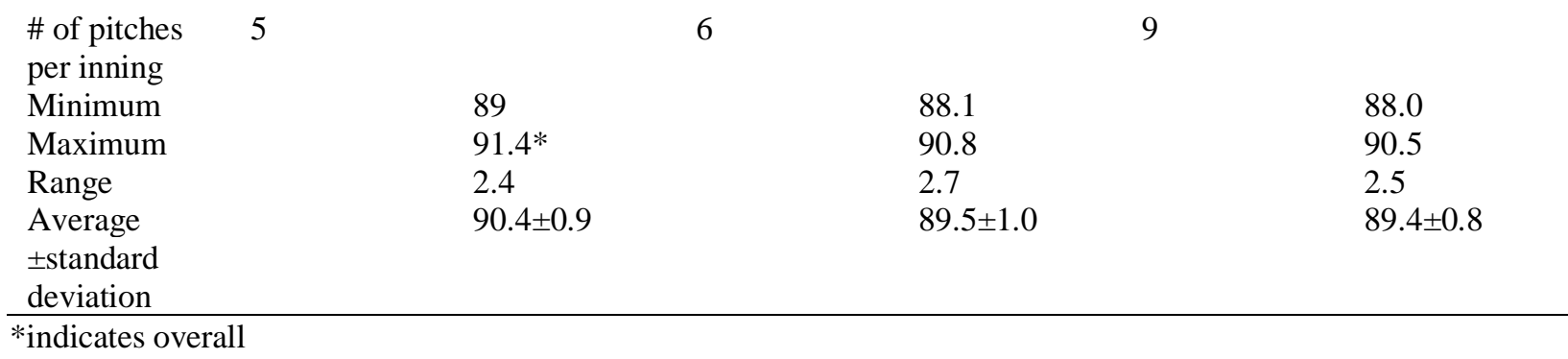

Table D20. Fastball Pitch Count, Velocity Chart, Descriptive Statistics Continued.

Game \#: 12

Subject \#: 2

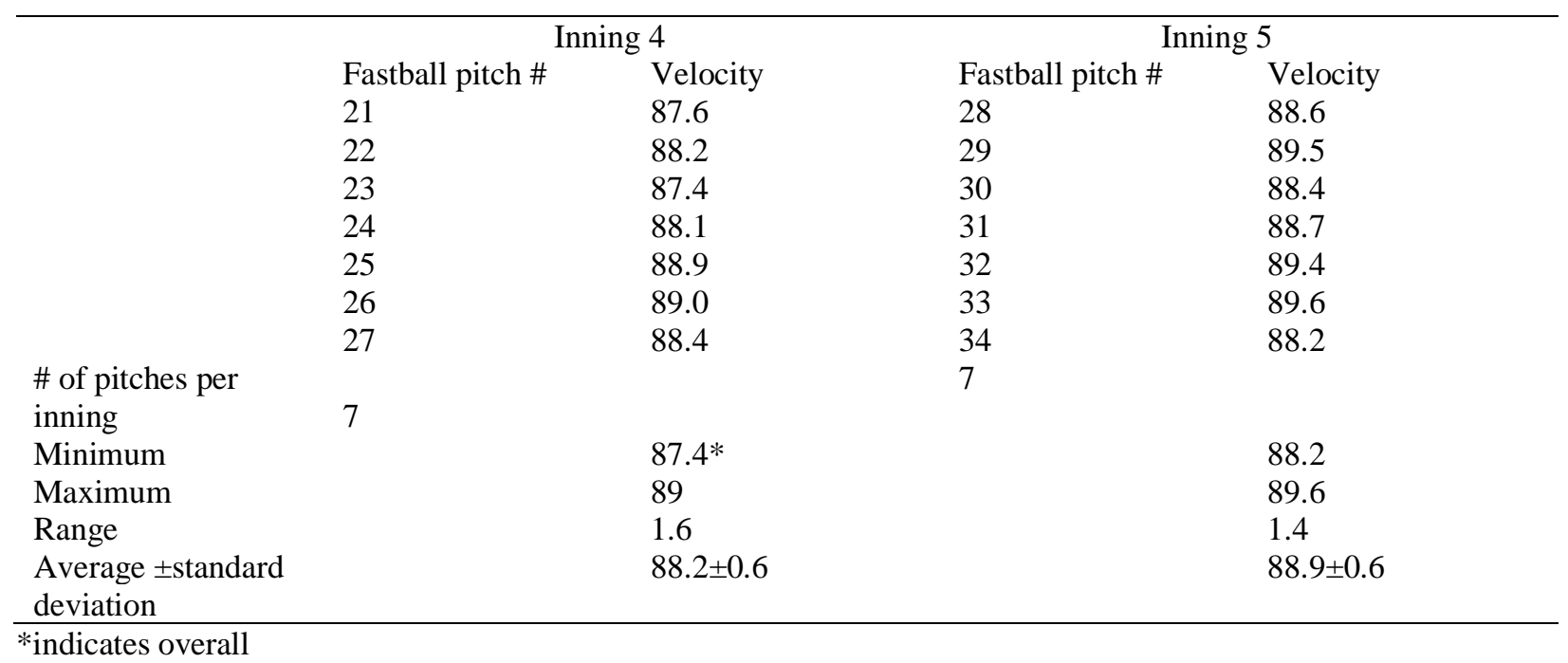

Table D21. Fastball Pitch Count, Velocity Chart, Descriptive Statistics.

Game \#: 13

Subject \#: 3

\begin{tabular}{|c|c|c|c|c|c|}
\hline \multicolumn{2}{|c|}{ Inning 1} & \multicolumn{2}{|c|}{ Inning 2} & \multicolumn{2}{|c|}{ Inning 3} \\
\hline $\begin{array}{l}\text { Fastball pitch } \\
\#\end{array}$ & Velocity & $\begin{array}{l}\text { Fastball pitch } \\
\#\end{array}$ & Velocity & $\begin{array}{l}\text { Fastball pitch } \\
\#\end{array}$ & Velocity \\
\hline 1 & 93.0 & 20 & 92.7 & 32 & 92.1 \\
\hline 2 & 93.0 & 21 & 92.3 & 33 & 92.2 \\
\hline 3 & 94.9 & 22 & 93.0 & 34 & 92.4 \\
\hline 4 & 93.4 & 23 & 92.0 & 35 & 92.0 \\
\hline 5 & 92.9 & 24 & 93.6 & 36 & 92.3 \\
\hline 6 & 93.3 & 25 & 91.7 & 37 & 90.8 \\
\hline 7 & 93.0 & 26 & 93.3 & 38 & 92.0 \\
\hline 8 & 94.5 & 27 & 93.4 & 39 & 93.2 \\
\hline 9 & 93.7 & 28 & 92.6 & & \\
\hline 10 & 93.9 & 29 & 92.8 & & \\
\hline 11 & 93.8 & 30 & 91.8 & & \\
\hline 12 & 93.2 & 31 & 94.0 & & \\
\hline 13 & 93.0 & & & & \\
\hline
\end{tabular}




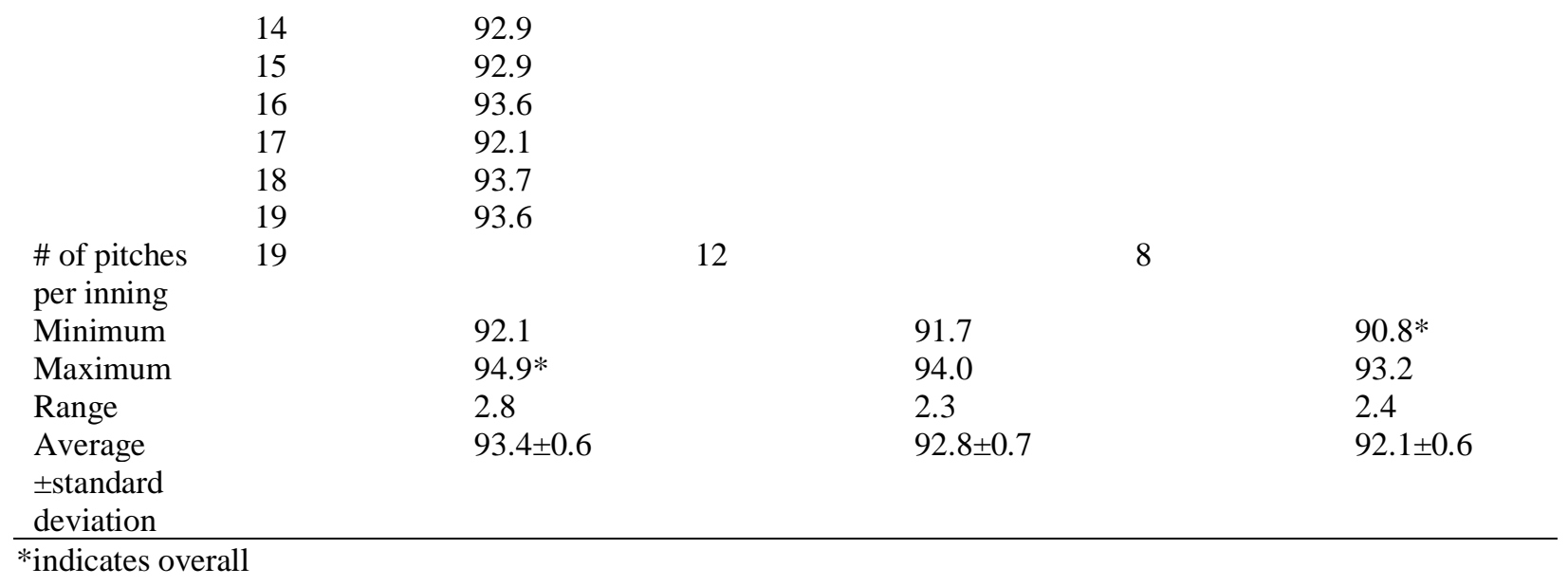

$\underline{\text { Table D22. Fastball Pitch Count, Velocity Chart, Descriptive Statistics Continued. }}$

Game \#: 13

Subject \#: 3

\begin{tabular}{|c|c|c|c|c|}
\hline & \multicolumn{2}{|c|}{ Inning 4} & \multicolumn{2}{|c|}{ Inning 5} \\
\hline & Fastball pitch \# & Velocity & Fastball pitch \# & Velocity \\
\hline & 40 & 90.8 & 48 & 91.2 \\
\hline & 41 & 91.8 & 49 & 92.4 \\
\hline & 42 & 91.7 & 50 & 92.1 \\
\hline & 43 & 92.3 & 51 & 91.4 \\
\hline & 44 & 92.0 & 52 & 92.0 \\
\hline & 45 & 92.1 & 53 & 91.0 \\
\hline & 46 & 92.6 & 54 & 92.0 \\
\hline & 47 & 91.6 & 55 & 91.3 \\
\hline & & & 56 & 92.2 \\
\hline & & & 57 & 91.4 \\
\hline & & & 58 & 92.4 \\
\hline \# of pitches per & & & 11 & \\
\hline inning & 8 & & & \\
\hline Minimum & & $90.8^{*}$ & & 91.0 \\
\hline Maximum & & 92.6 & & 92.4 \\
\hline Range & & 1.8 & & 1.4 \\
\hline $\begin{array}{l}\text { Average } \pm \text { standard } \\
\text { deviation }\end{array}$ & & $91.9 \pm 0.5$ & & $91.8 \pm 0.5$ \\
\hline
\end{tabular}

Table D23. Fastball Pitch Count, Velocity Chart, Descriptive Statistics.

Game \#: 14

Subject \#: 1

\begin{tabular}{llllllllll}
\multicolumn{2}{c}{ Inning 1 } & \multicolumn{2}{c}{ Inning 2 } & \multicolumn{2}{c}{ Inning 3 } & \multicolumn{2}{c}{ Inning 4 } & \multicolumn{2}{c}{ Inning 5 } \\
$\begin{array}{l}\text { Fastball } \\
\text { pitch \# }\end{array}$ & Velocity & $\begin{array}{l}\text { Fastball } \\
\text { pitch \# }\end{array}$ & Velocity & $\begin{array}{l}\text { Fastball } \\
\text { pitch \# }\end{array}$ & Velocity & Fastball & Velocity & Fastball & Velocity \\
1 & 92.5 & 12 & 93.7 & 22 & 93.3 & 27 & 93.8 & 34 & 93.4 \\
2 & 93.1 & 13 & 94.6 & 23 & 93.3 & 28 & 94.8 & 35 & 93.3 \\
3 & 94.7 & 14 & 94.6 & 24 & 94.7 & 29 & 95.1 & 36 & 94.4
\end{tabular}




$\begin{array}{llllllllll}4 & 94.3 & 15 & 95.2 & 25 & 93.3 & 30 & 93.7 & 37 & 93.7 \\ 5 & 94.6 & 16 & 95.7 & 26 & 93.2 & 31 & 95.1 & 38 & 94.7 \\ 6 & 95.2 & 17 & 95.5 & & & 32 & 94.3 & 39 & 94.8 \\ 7 & 95.3 & 18 & 91.9 & & & 33 & 94.3 & & \\ 8 & 95.4 & 19 & 92.9 & & & & & & \\ 9 & 94.3 & 20 & 94.6 & & & & & & \\ 10 & 93.9 & 21 & 93.6 & & & & & \end{array}$

\begin{tabular}{|c|c|c|c|c|c|}
\hline $\begin{array}{l}\text { \# of } \\
\text { pitches per } \\
\text { inning }\end{array}$ & & & & & \\
\hline Minimum & 92.5 & $91.9 *$ & 93.2 & 93.7 & 93.3 \\
\hline Maximum & 95.4 & 95.7 & 94.7 & 95.1 & 94.8 \\
\hline Range & 2.9 & 3.8 & 1.5 & 1.4 & 1.5 \\
\hline $\begin{array}{l}\text { Average } \pm \\
\text { standard } \\
\text { deviation }\end{array}$ & $94.4 \pm 0.9$ & $94.2 \pm 1.2$ & $93.6 \pm 0.6$ & $94.4 \pm 0.6$ & $94.0 \pm 0.7$ \\
\hline
\end{tabular}

Table D24. Fastball Pitch Count, Velocity Chart, Descriptive Statistics Continued.

Game \#: 14

Subject \#: 1

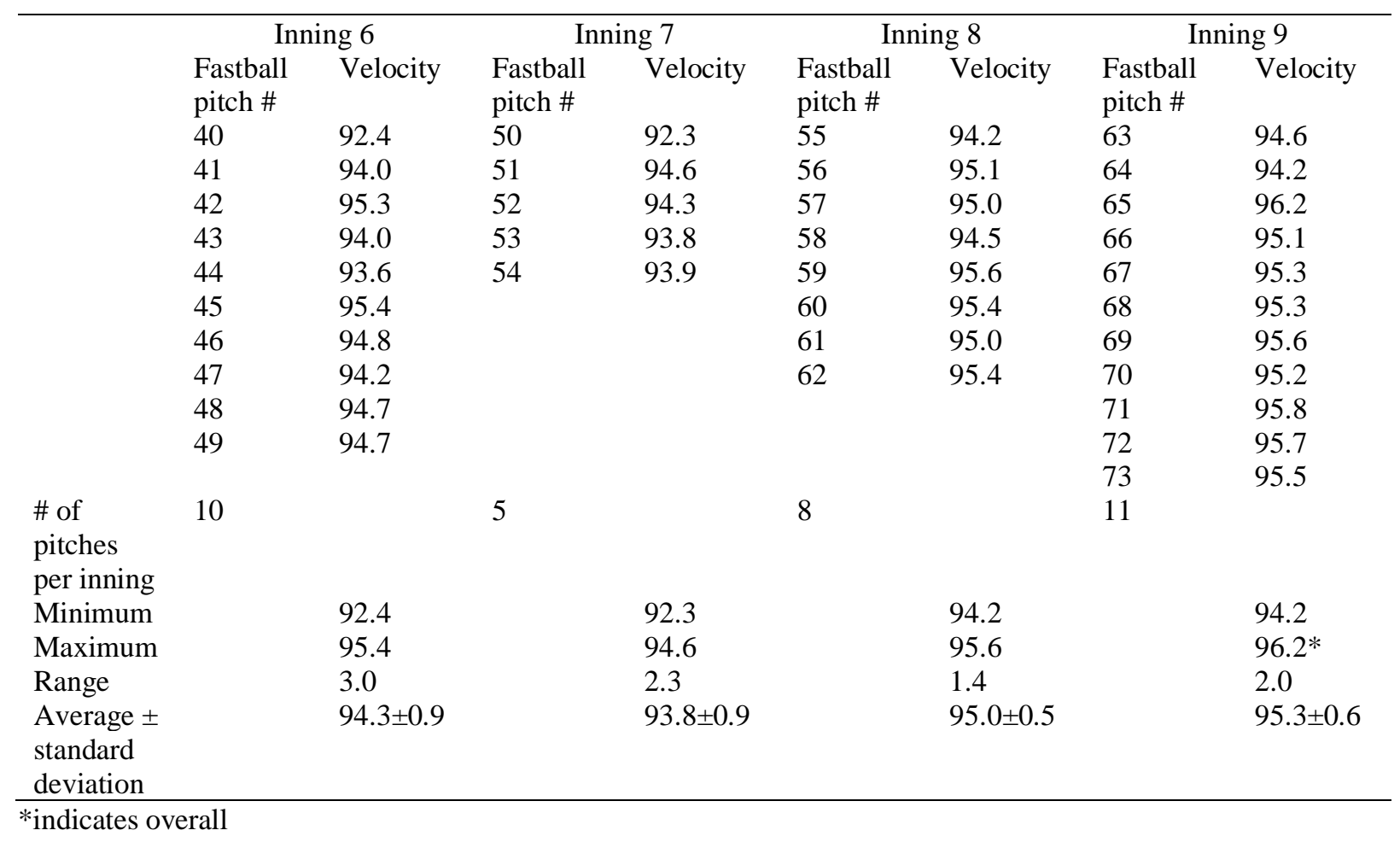

Table D25. Fastball Pitch Count, Velocity Chart, Descriptive Statistics.

Game \#: 15 
Subject \#: 2

\begin{tabular}{|c|c|c|c|c|c|c|}
\hline & \multicolumn{2}{|c|}{ Inning 1} & \multicolumn{2}{|c|}{ Inning 2} & \multicolumn{2}{|c|}{ Inning 3} \\
\hline & $\begin{array}{l}\text { Fastball pitch } \\
\#\end{array}$ & Velocity & $\begin{array}{l}\text { Fastball pitch } \\
\#\end{array}$ & Velocity & $\begin{array}{l}\text { Fastball pitch } \\
\#\end{array}$ & Velocity \\
\hline & 1 & 90.6 & 10 & 86.8 & 17 & 85.8 \\
\hline & 2 & 89.7 & 11 & 88.7 & 18 & 87.7 \\
\hline & 3 & 89.5 & 12 & 88.9 & 19 & 84.1 \\
\hline & 4 & 90.2 & 13 & 89.4 & 20 & 87.3 \\
\hline & 5 & 90.5 & 14 & 88.7 & 21 & 87.9 \\
\hline & 6 & 90.0 & 15 & 90.7 & 22 & 87.8 \\
\hline & 7 & 91.5 & 16 & 89.3 & 23 & 88.0 \\
\hline & 8 & 89.5 & & & & \\
\hline & 9 & 90.4 & & & & \\
\hline $\begin{array}{l}\text { \# of pitches } \\
\text { per inning }\end{array}$ & 9 & & 7 & & 7 & \\
\hline Minimum & & 89.5 & & 86.8 & & 84.1 \\
\hline Maximum & & $91.5^{*}$ & & 90.7 & & 88.0 \\
\hline Range & & 2.0 & & 3.9 & & 3.9 \\
\hline $\begin{array}{l}\text { Average } \\
\pm \text { standard } \\
\text { deviation }\end{array}$ & & $90.2 \pm 0.6$ & & $88.9 \pm 1.2$ & & $86.9 \pm 1.5$ \\
\hline
\end{tabular}

Table D26. Fastball Pitch Count, Velocity Chart, Descriptive Statistics Continued.

Game \#: 15

Subject \#: 2

\begin{tabular}{|c|c|c|c|c|c|c|}
\hline & \multicolumn{2}{|c|}{ Inning 4} & \multicolumn{2}{|c|}{ Inning 5} & \multicolumn{2}{|c|}{ Inning 6} \\
\hline & $\begin{array}{l}\text { Fastball pitch } \\
\#\end{array}$ & Velocity & $\begin{array}{l}\text { Fastball pitch } \\
\#\end{array}$ & Velocity & $\begin{array}{l}\text { Fastball pitch } \\
\#\end{array}$ & Velocity \\
\hline & 24 & 87.6 & 32 & 86.6 & 41 & 87.7 \\
\hline & 25 & 87.3 & 33 & 87.7 & 42 & 88.7 \\
\hline & 26 & 88.5 & 34 & 83.7 & 43 & 87.8 \\
\hline & 27 & 88.0 & 35 & 85.4 & 44 & 88.8 \\
\hline & 28 & 87.8 & 36 & 88.3 & 45 & 84.7 \\
\hline & 29 & 88.1 & 37 & 88.6 & 46 & 84.3 \\
\hline & 30 & 88.9 & 38 & 88.3 & 47 & 84.6 \\
\hline & 31 & 87.2 & 39 & 89.0 & 48 & 88.1 \\
\hline & & & 40 & 88.0 & 49 & 87.6 \\
\hline & & & & & 50 & 88.8 \\
\hline $\begin{array}{l}\text { \# of pitches } \\
\text { per inning }\end{array}$ & 8 & & 9 & & 10 & \\
\hline Minimum & & 87.2 & & $83.7 *$ & & 84.3 \\
\hline Maximum & & 88.9 & & 89.0 & & 88.8 \\
\hline Range & & 1.2 & & 5.3 & & 4.5 \\
\hline $\begin{array}{l}\text { Average } \\
\pm \text { standard } \\
\text { deviation }\end{array}$ & & $87.9 \pm 0.58$ & & $87.3 \pm 1.7$ & & $87.1 \pm 1.8$ \\
\hline
\end{tabular}

Table D27. Fastball Pitch Count, Velocity Chart, Descriptive Statistics.

Game \#: 15 
Subject \#: 4

\begin{tabular}{|c|c|c|c|c|c|c|}
\hline & \multicolumn{2}{|c|}{ Inning 7} & \multicolumn{2}{|c|}{ Inning 8} & \multicolumn{2}{|c|}{ Inning 9} \\
\hline & $\begin{array}{l}\text { Fastball pitch } \\
\#\end{array}$ & Velocity & $\begin{array}{l}\text { Fastball pitch } \\
\#\end{array}$ & Velocity & $\begin{array}{l}\text { Fastball pitch } \\
\#\end{array}$ & Velocity \\
\hline & 1 & 91.1 & 16 & 89.1 & 31 & 88.5 \\
\hline & 2 & 90.7 & 17 & 91.3 & 32 & 89.4 \\
\hline & 3 & 89.4 & 18 & 90.2 & 33 & 89.7 \\
\hline & 4 & 90.4 & 19 & 89.7 & 34 & 89.0 \\
\hline & 5 & 90.2 & 20 & 90.6 & 35 & 90.1 \\
\hline & 6 & 90.7 & 21 & 91.7 & 36 & 89.2 \\
\hline & 7 & 90.1 & 22 & 89.2 & 37 & 89.1 \\
\hline & 8 & 90.1 & 23 & 91.2 & 38 & 87.0 \\
\hline & 9 & 90.7 & 24 & 91.4 & 39 & 90.8 \\
\hline & 10 & 91.0 & 25 & 91.3 & 40 & 89.7 \\
\hline & 11 & 90.2 & 26 & 92.7 & 41 & 89.8 \\
\hline & 12 & 90.2 & 27 & 90.7 & 42 & 89.6 \\
\hline & 13 & 91.2 & 28 & 92.3 & & \\
\hline & 14 & 91.2 & 29 & 92.5 & & \\
\hline & 15 & 90.9 & 30 & 91.9 & & \\
\hline $\begin{array}{l}\text { \# of pitches } \\
\text { per inning }\end{array}$ & & & & & & \\
\hline Minimum & & 89.4 & & 89.1 & & $87.0 *$ \\
\hline Maximum & & 91.2 & & $92.7 *$ & & 90.8 \\
\hline Range & & 1.8 & & 3.6 & & 3.8 \\
\hline $\begin{array}{l}\text { Average } \\
\pm \text { standard } \\
\text { deviation }\end{array}$ & & $90.5 \pm 0.5$ & & $91.1 \pm 1.1$ & & $89.3 \pm 0.9$ \\
\hline
\end{tabular}

Table D28. Fastball Pitch Count, Velocity Chart, Descriptive Statistics.

Game \#: 16

Subject \#: 3 


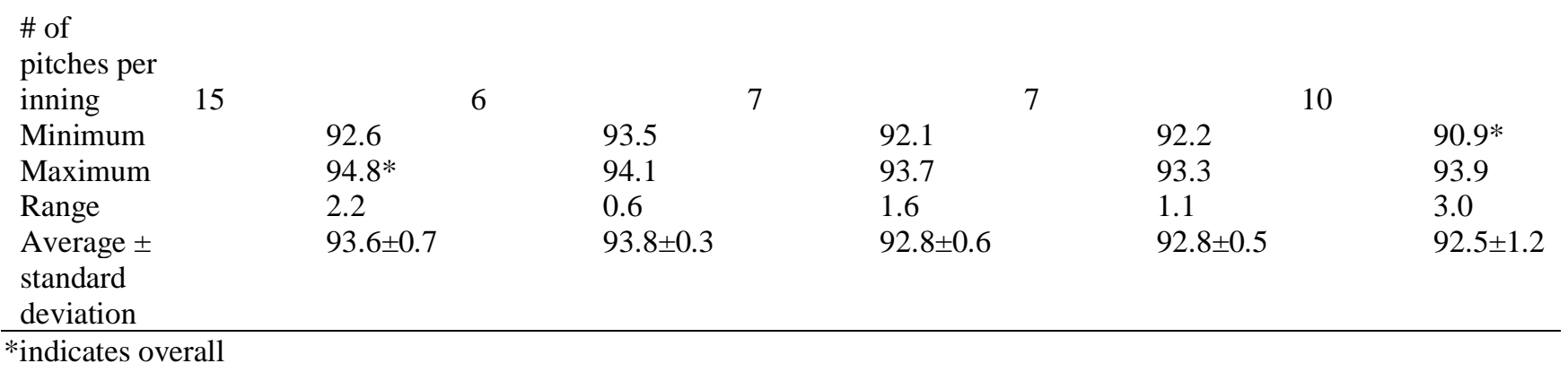

\section{Table D29. Fastball Pitch Count, Velocity Chart, Descriptive Statistics.}

Game \#: 17

Subject \#: 3

\begin{tabular}{|c|c|c|c|c|c|c|}
\hline & & $\lg 3$ & $\mathrm{Inn}$ & $\lg 5$ & & $\lg 6$ \\
\hline & $\begin{array}{l}\text { Fastball pitch } \\
\#\end{array}$ & Velocity & $\begin{array}{l}\text { Fastball pitch } \\
\#\end{array}$ & Velocity & $\begin{array}{l}\text { Fastball pitch } \\
\#\end{array}$ & Velocity \\
\hline & 45 & 93.2 & 52 & 92.0 & 59 & 92.3 \\
\hline & 46 & 93.3 & 53 & 91.4 & 60 & 92.9 \\
\hline & 47 & 93.6 & 54 & 92.2 & 61 & 93.1 \\
\hline & 48 & 93.5 & 55 & 91.1 & 62 & 93.5 \\
\hline & 49 & 93.2 & 56 & 90.8 & 63 & 93.4 \\
\hline & 50 & 92.7 & 57 & 93.4 & 64 & 92.3 \\
\hline & 51 & 93.4 & 58 & 93.4 & 65 & 93.1 \\
\hline & & & & & 66 & 92.8 \\
\hline $\begin{array}{l}\text { \# of pitches } \\
\text { per inning }\end{array}$ & 7 & & 7 & & 8 & \\
\hline Minimum & & 92.7 & & $90.8^{*}$ & & 92.3 \\
\hline Maximum & & $93.6^{*}$ & & 93.4 & & 93.5 \\
\hline Range & & 0.7 & & 2.6 & & 1.2 \\
\hline $\begin{array}{l}\text { Average } \\
\pm \text { standard } \\
\text { deviation }\end{array}$ & & $93.3 \pm 0.3$ & & $92.0 \pm 1.1$ & & $92.9 \pm 0.5$ \\
\hline
\end{tabular}

\section{Figure D1. Game 1, Subject 4, Overall Fastball Pitch Velocity (mph).}

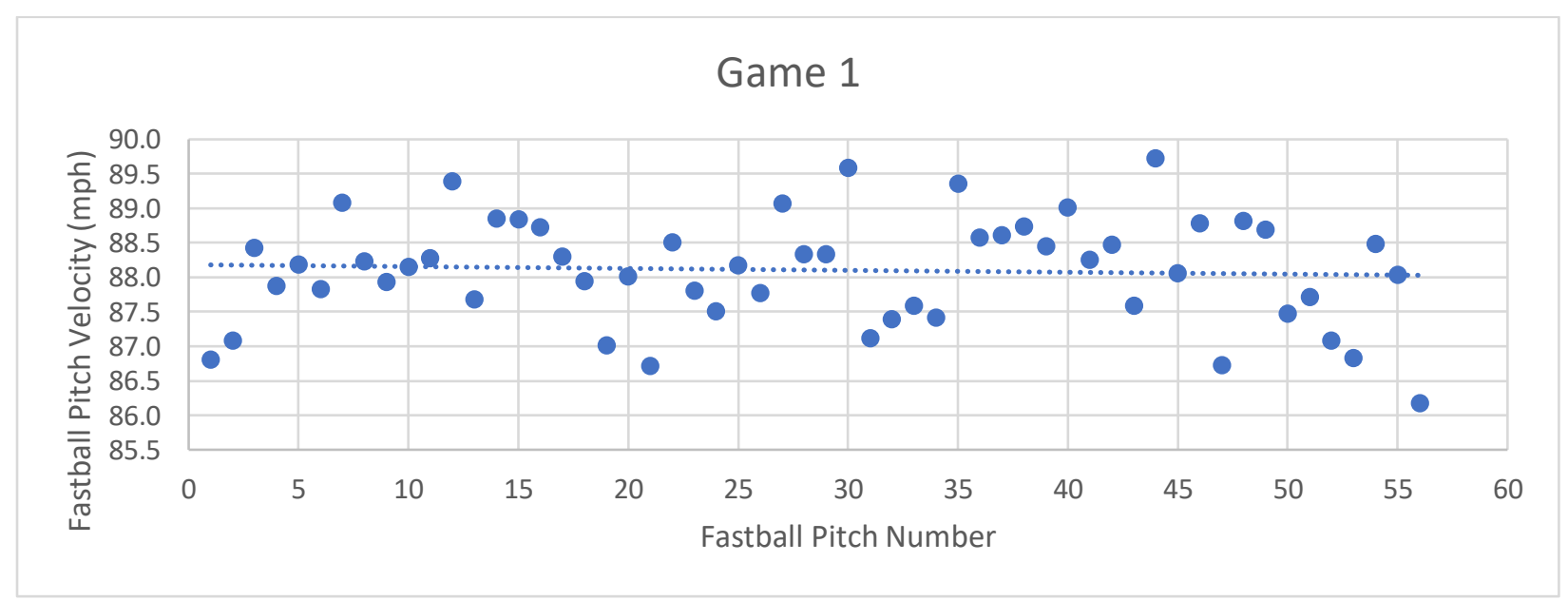


Figure D2. Game 1 Averages, Subject 4, Fastball Pitch Velocity Averages by Inning (mph).

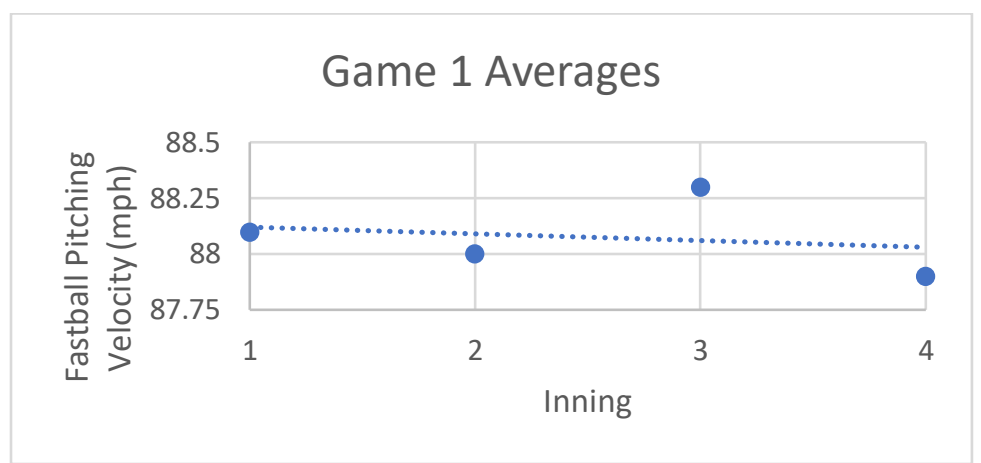

Figure D3. Game 2, Subject 4, Overall Fastball Pitch Velocity (mph).

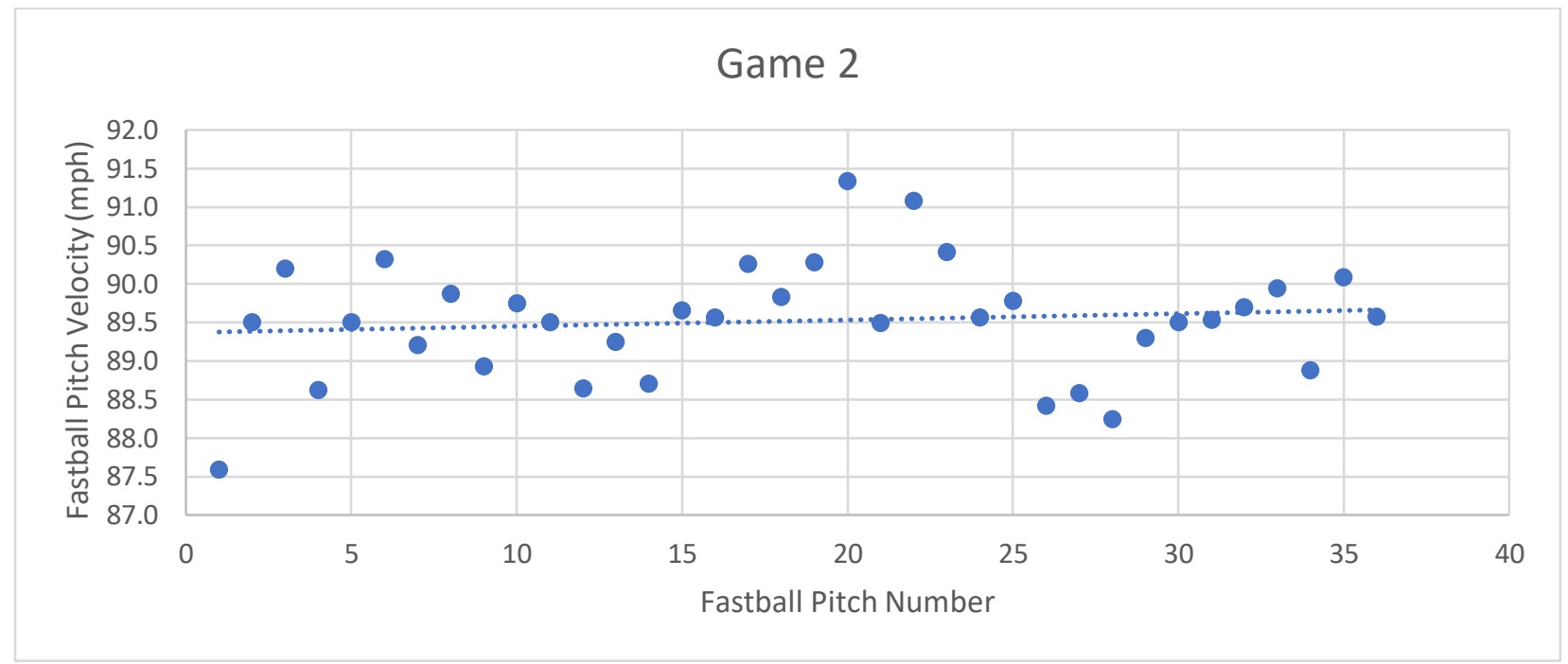

Figure D4. Game 2 Averages, Subject 4, Fastball Pitch Velocity Averages by Inning (mph).

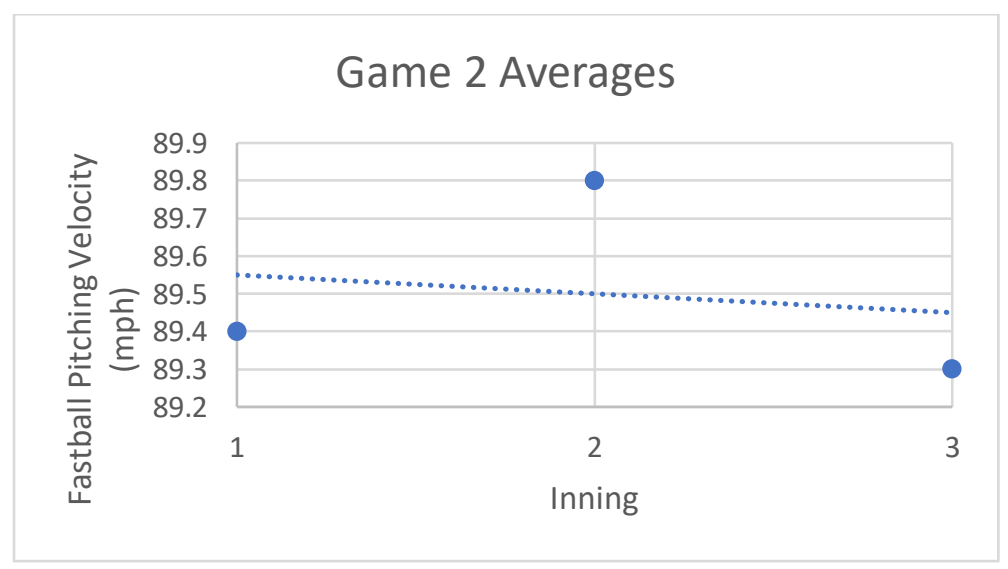


Figure D5. Game 3, Subject 1, Overall Fastball Pitch Velocity (mph).

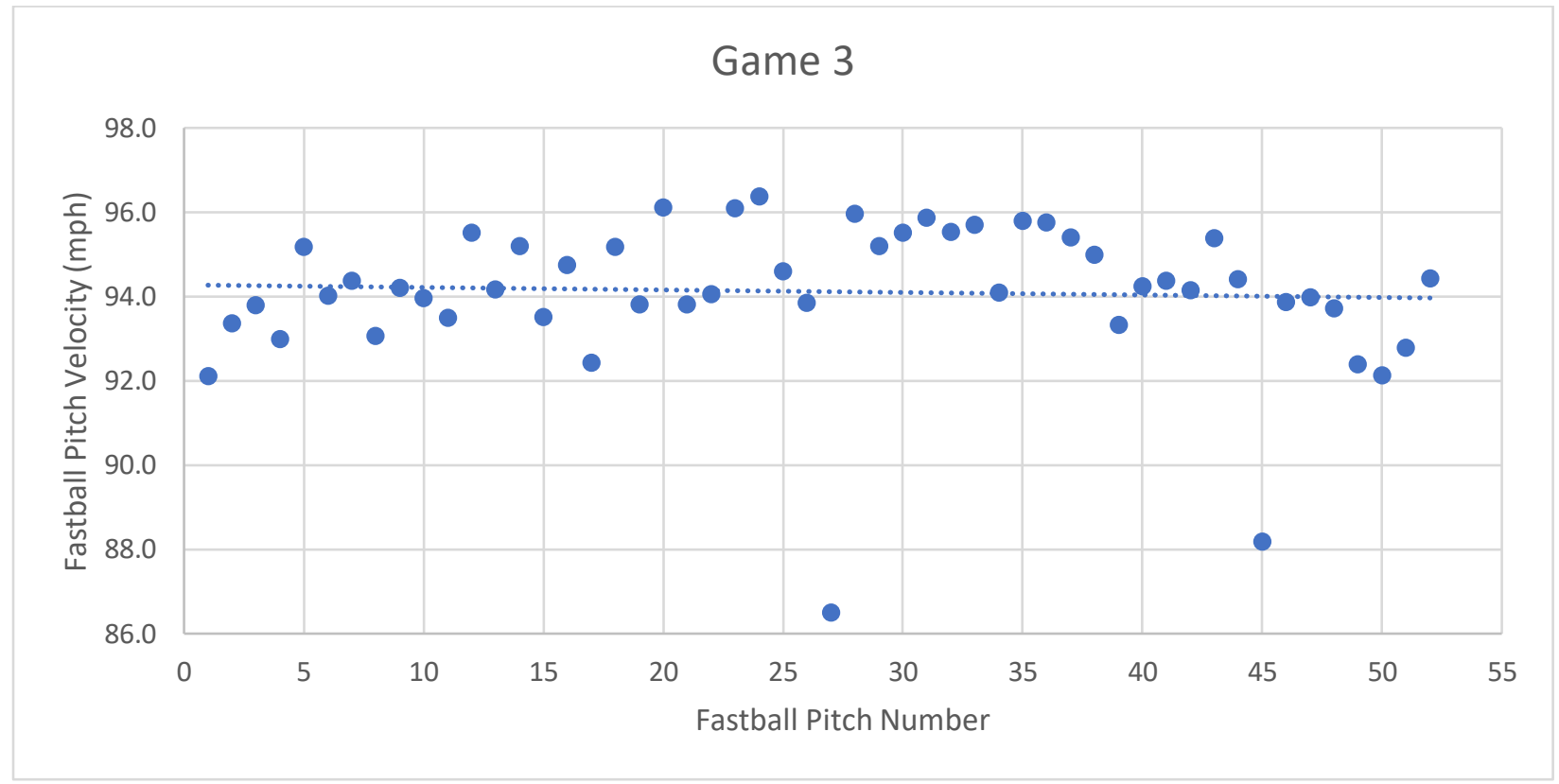

Figure D6. Game 3 Averages, Subject 1, Fastball Pitch Velocity Averages by Inning (mph).

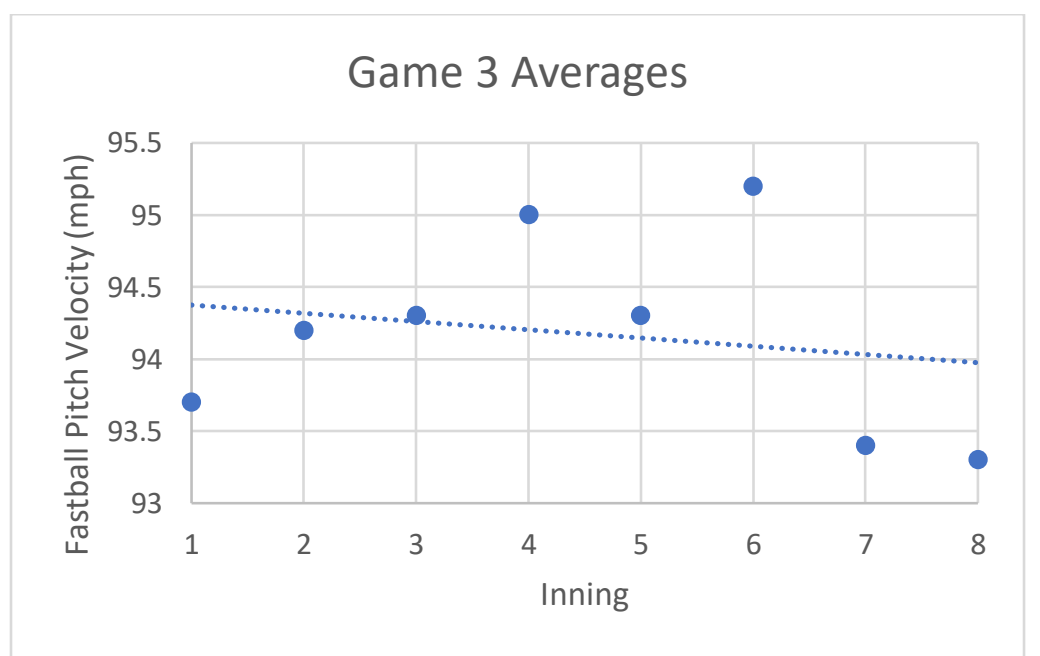


Figure D7. Game 4, Subject 2, Overall Fastball Pitch Velocity (mph).

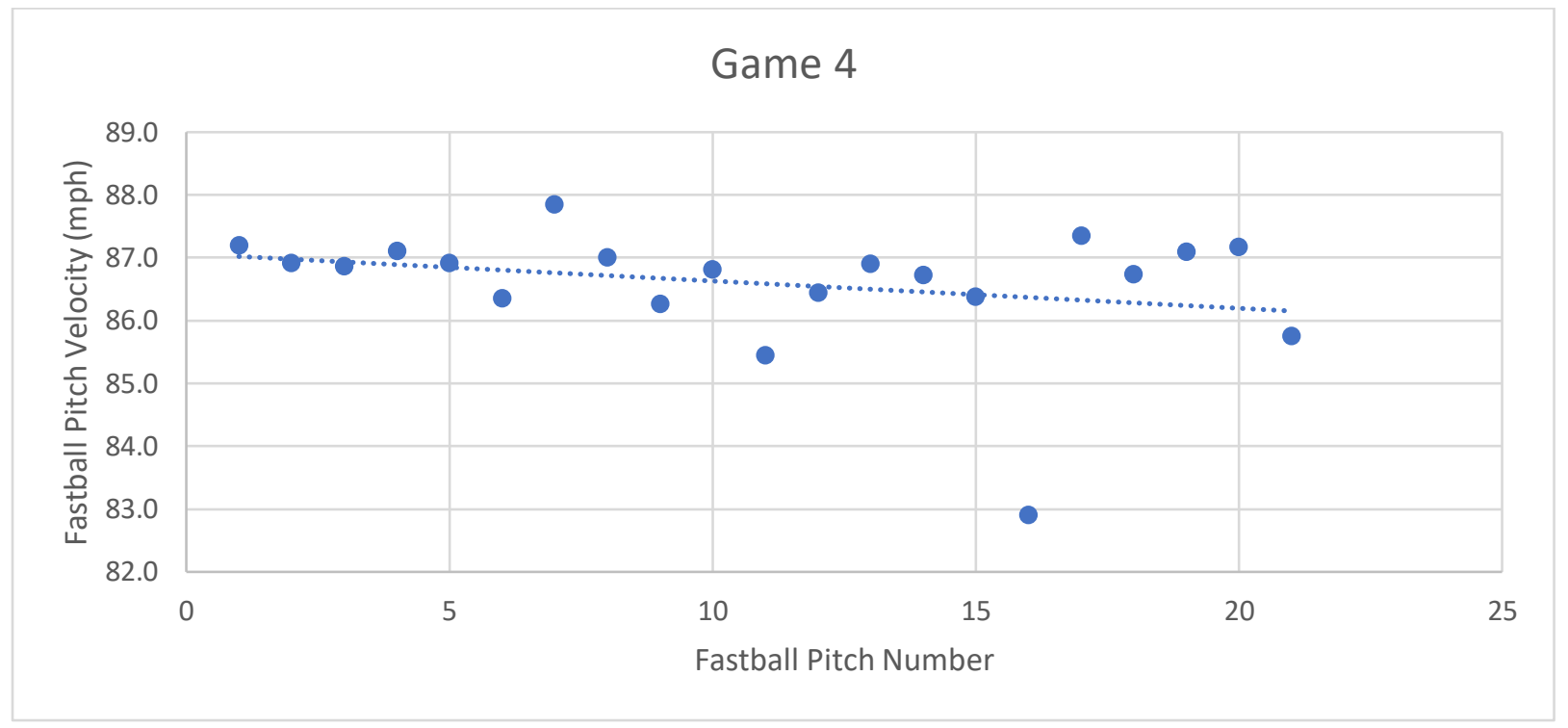

Figure D8. Game 4 Averages, Subject 2, Fastball Pitch Velocity Averages by Inning (mph).

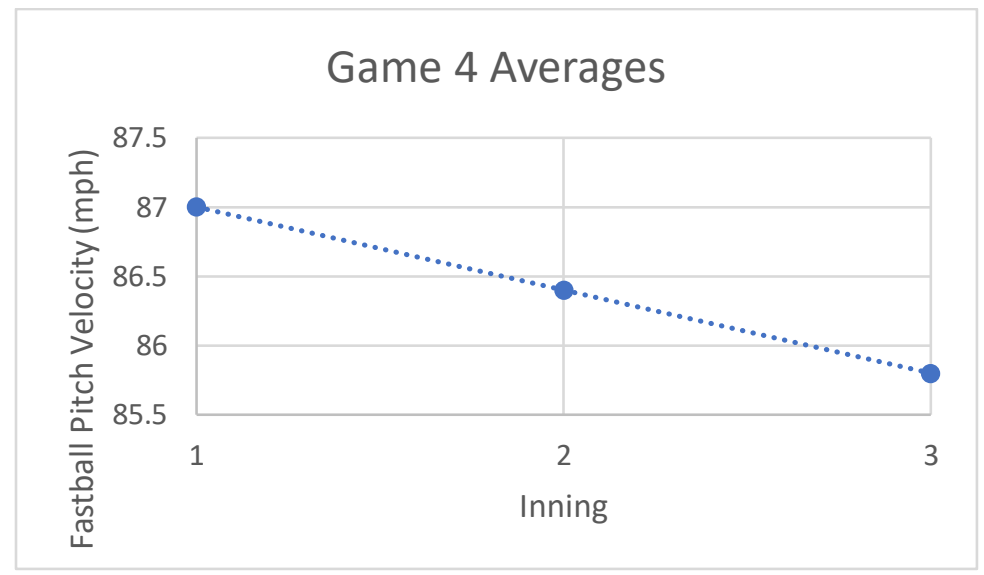


Figure D9. Game 5, Subject 3, Overall Fastball Pitch Velocity (mph).

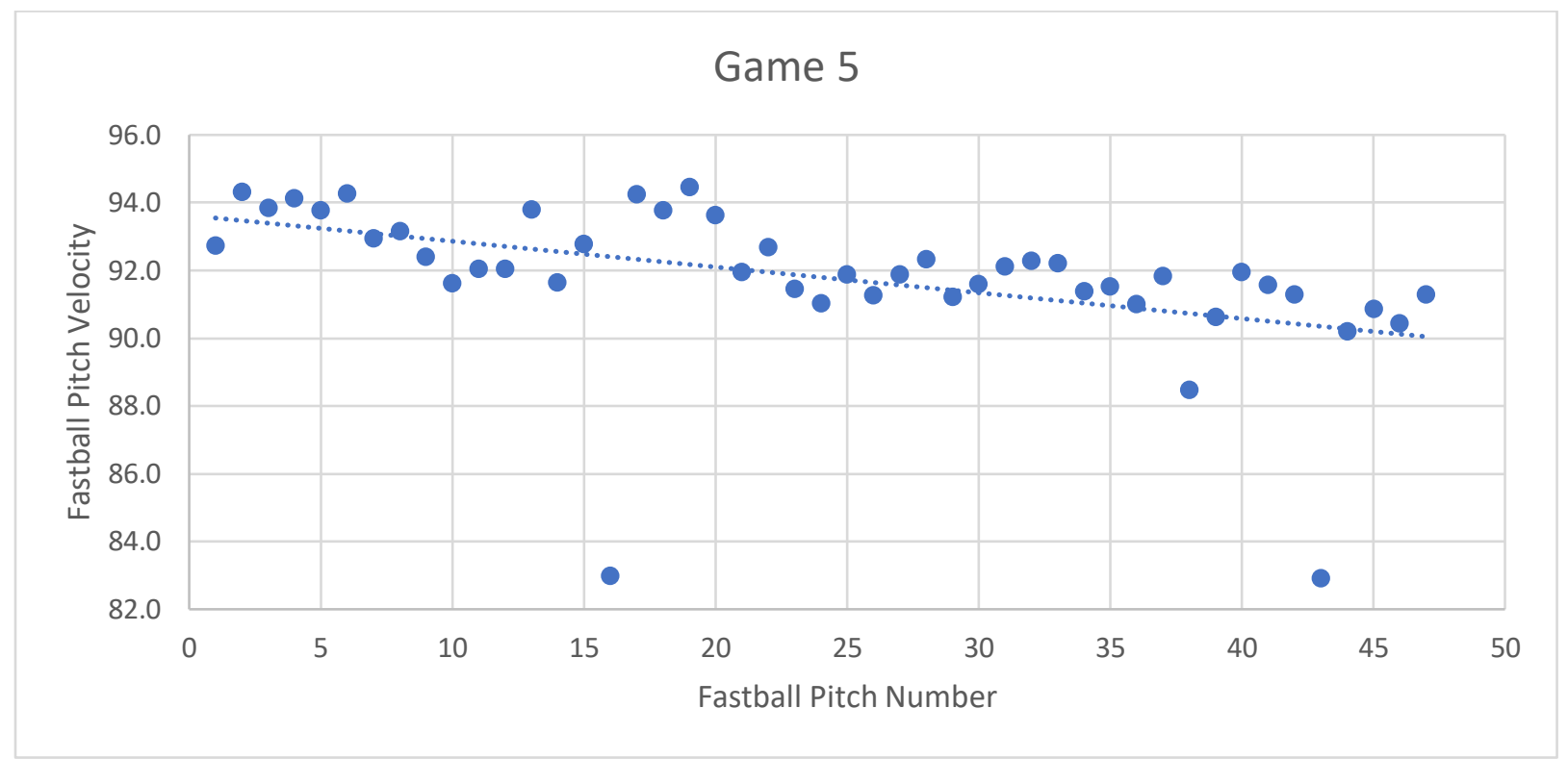

Figure D10. Game 5 Averages, Subject 3, Fastball Pitch Velocity Averages by Inning (mph).

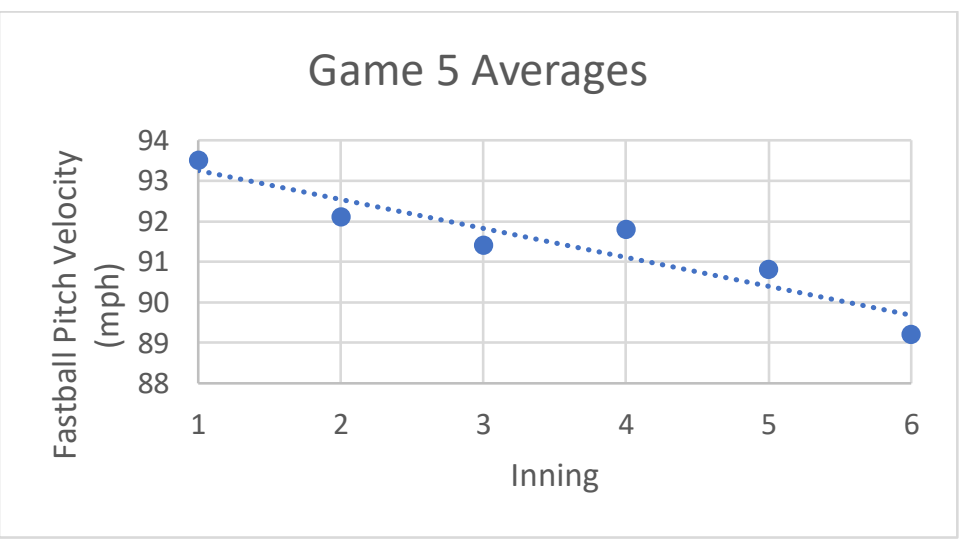


Figure D11. Game 6, Subject 4, Overall Fastball Pitch Velocity (mph).

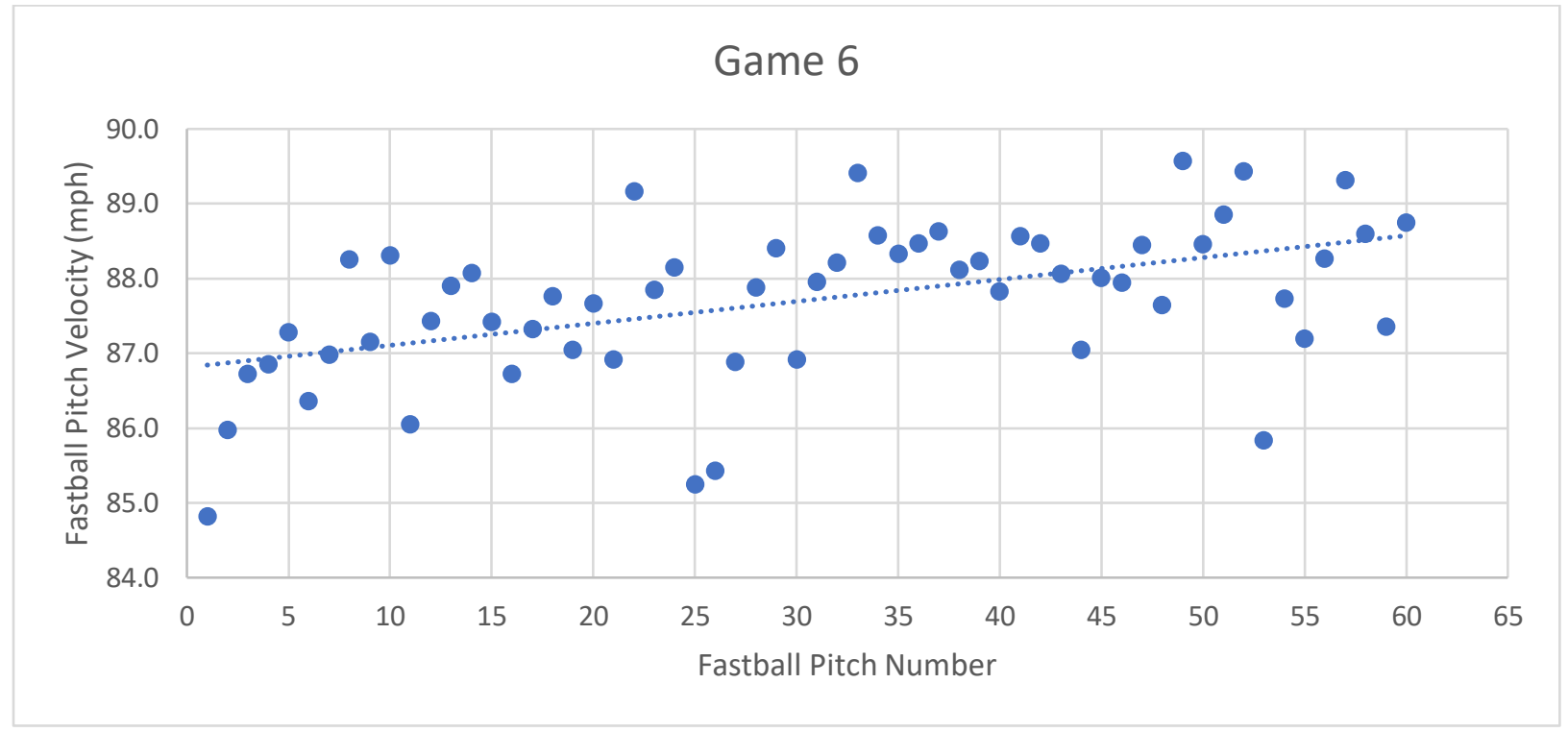

Figure D12. Game 6 Averages, Subject 4, Fastball Pitch Velocity Averages by Inning (mph).

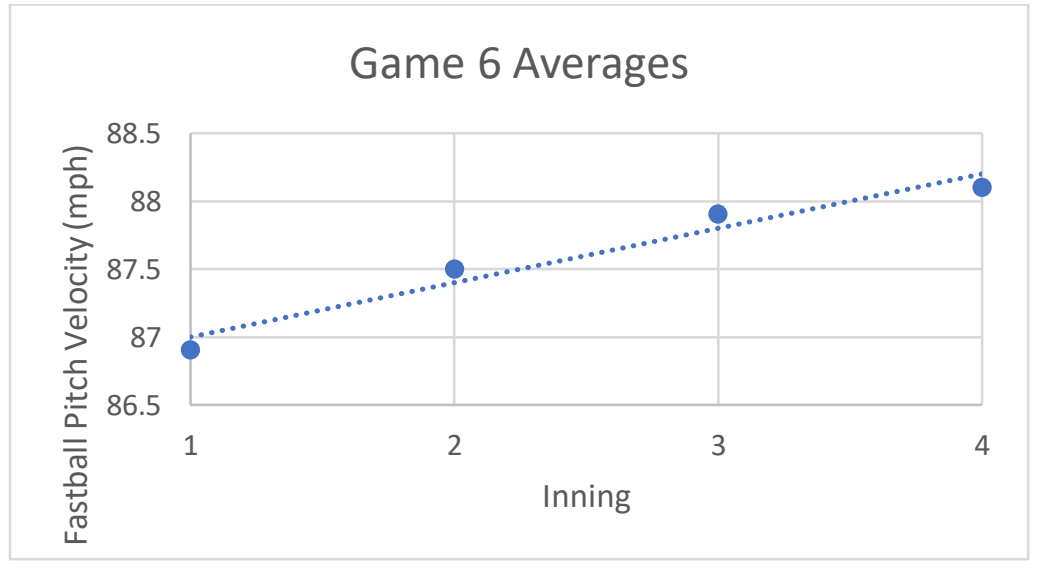


Figure D13. Game 7, Subject 1, Overall Fastball Pitch Velocity (mph).

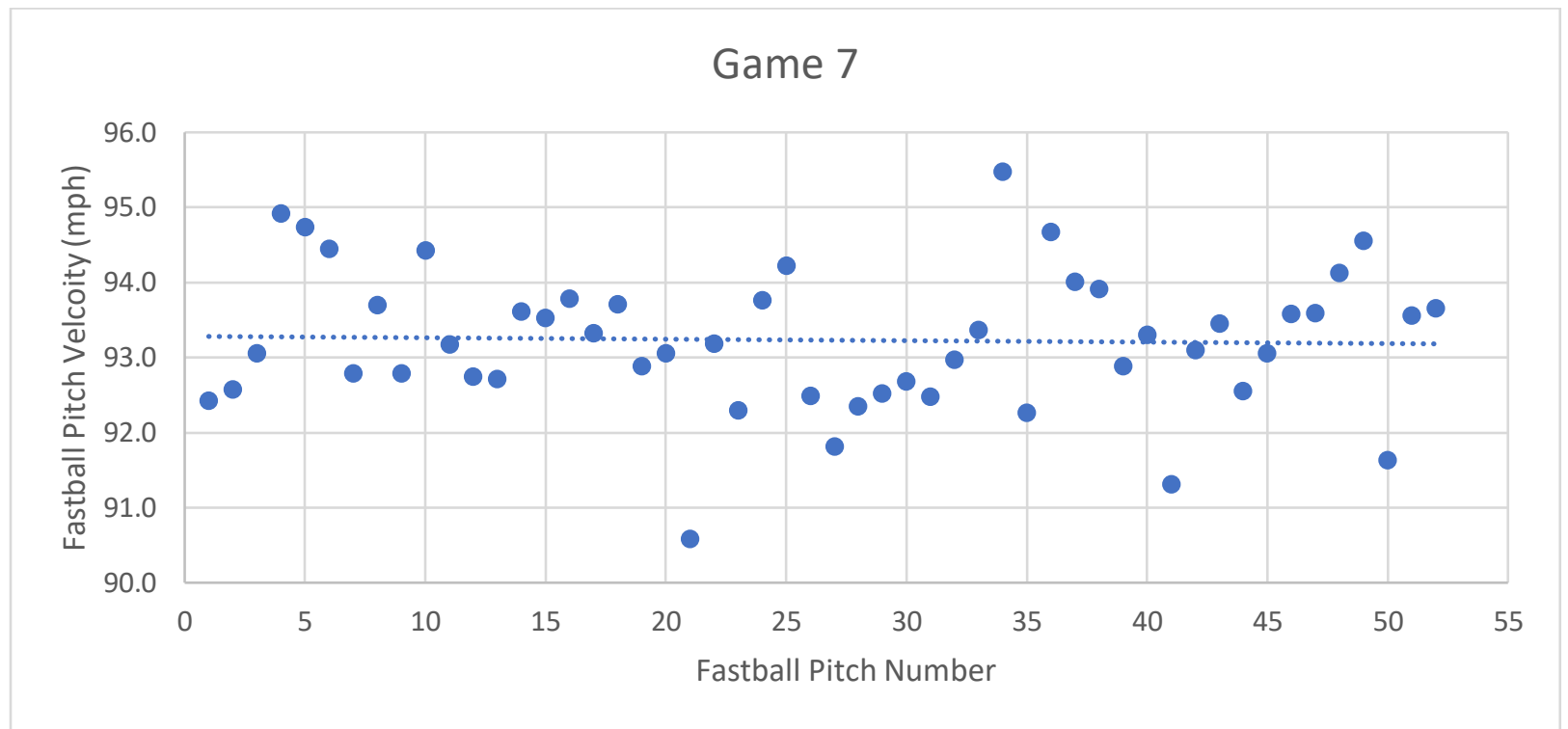

Figure D14. Game 7 Averages, Subject 1, Fastball Pitch Velocity Averages by Inning (mph).

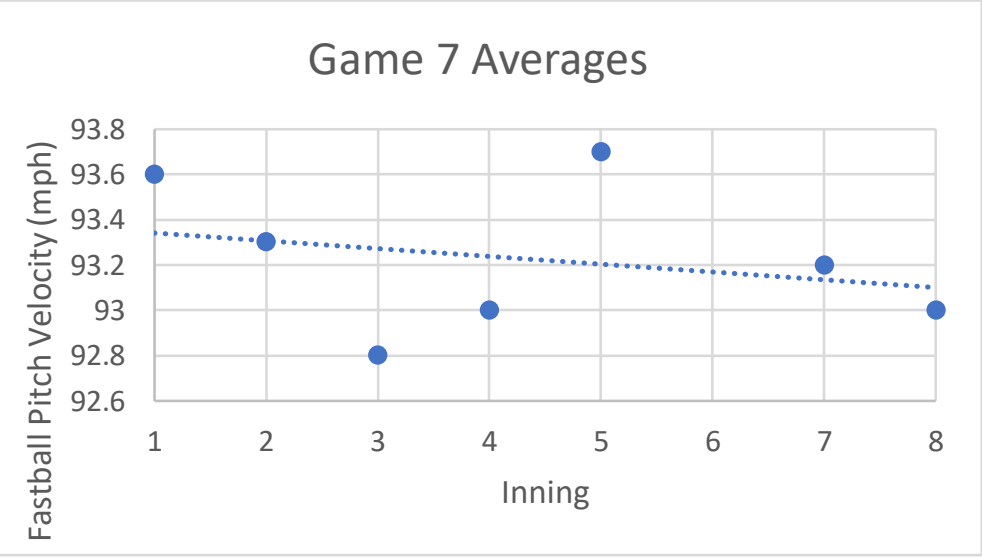


Figure D15. Game 8, Subject 2, Overall Fastball Pitch Velocity (mph).

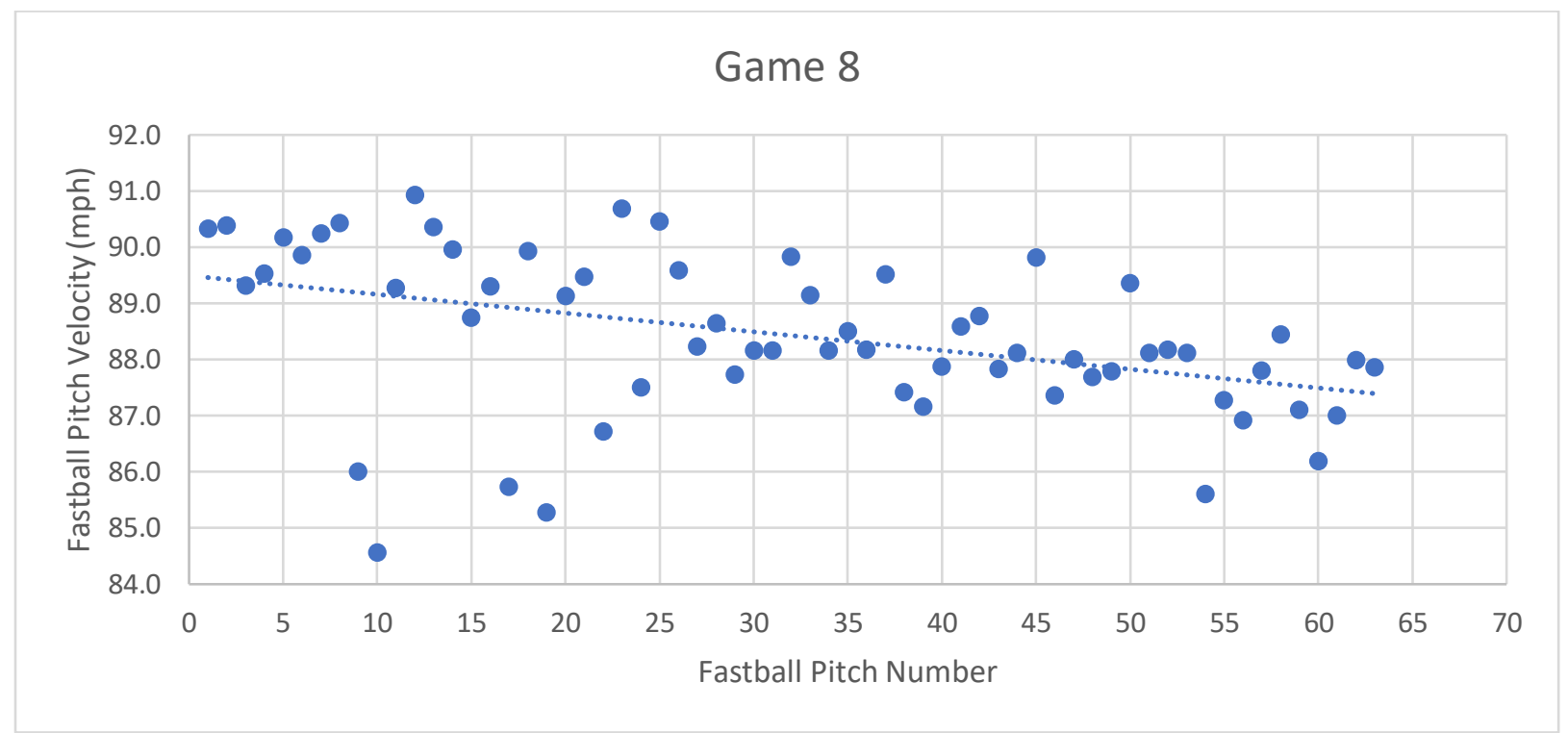

Figure D16. Game 8 Averages, Subject 2, Fastball Pitch Velocity Averages by Inning (mph).

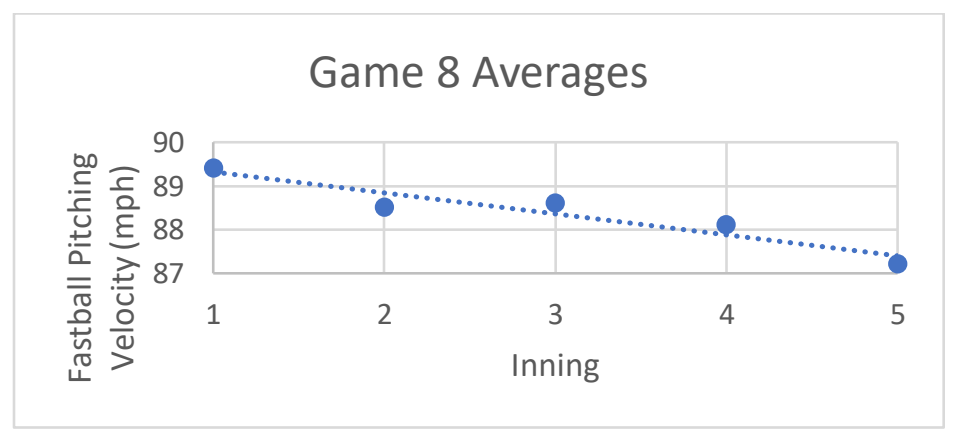

Figure D17. Game 9, Subject 3, Overall Fastball Pitch Velocity (mph).

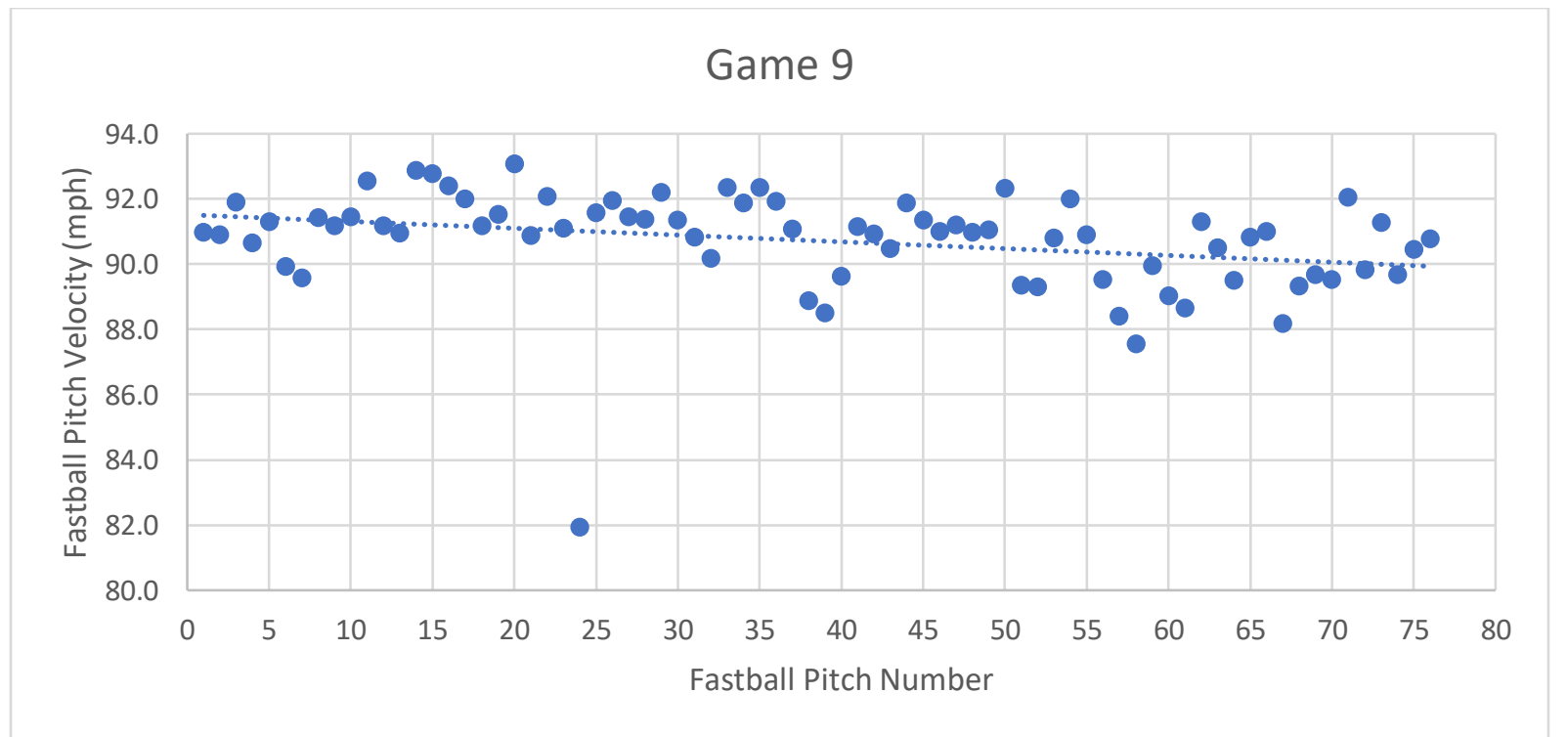


Figure D18. Game 9 Averages, Subject 3, Fastball Pitch Velocity Averages by Inning (mph).

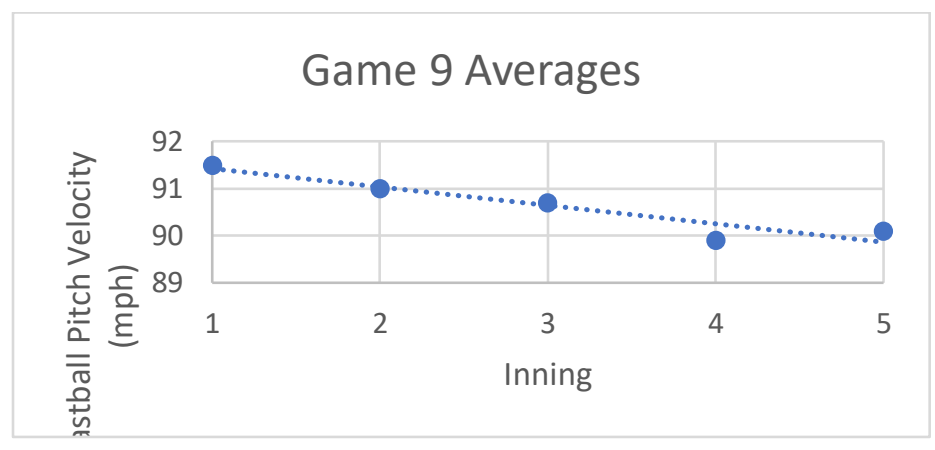

Figure D19. Game 10, Subject 4, Overall Fastball Pitch Velocity (mph).

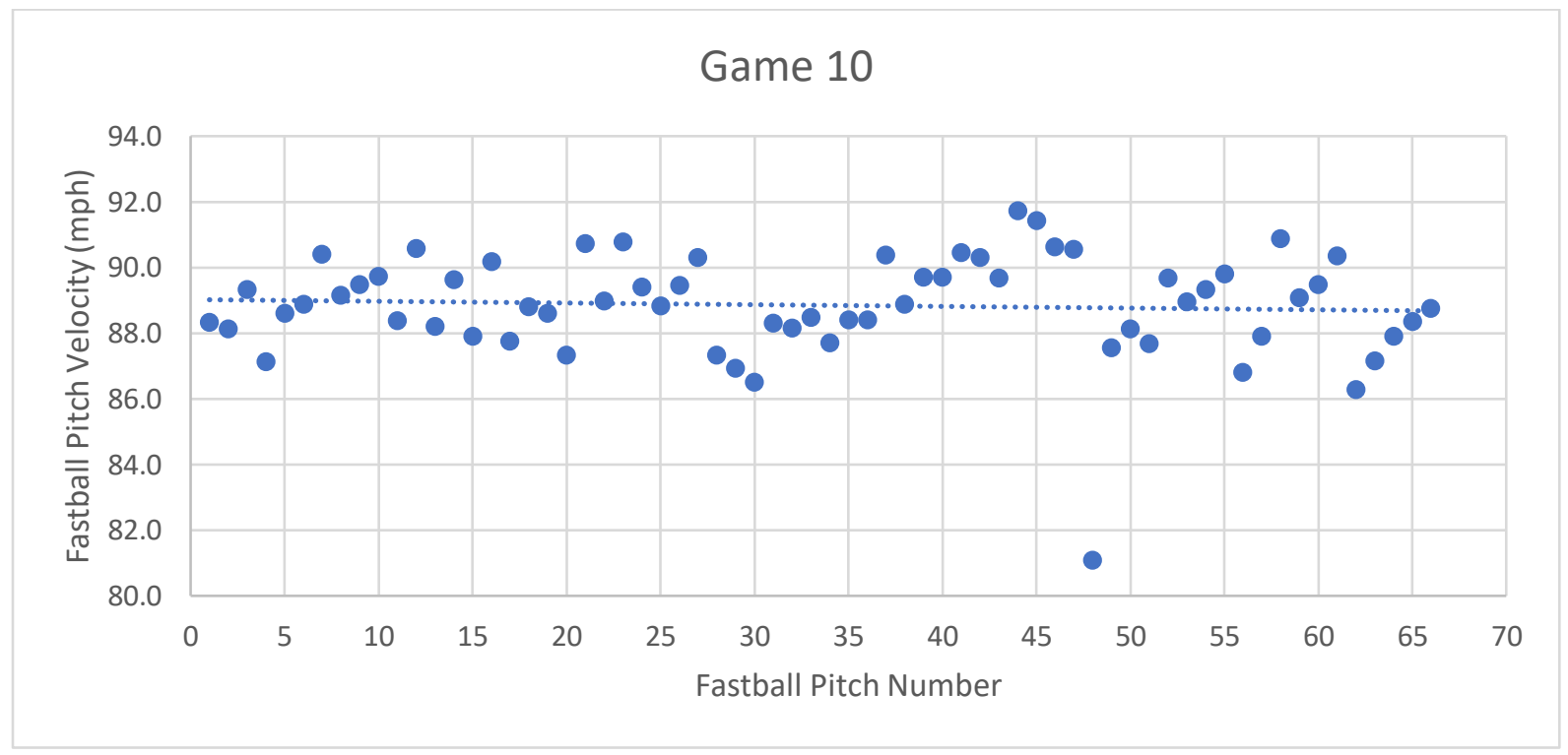

Figure D20. Game 10 Averages, Subject 4, Fastball Pitch Velocity Averages by Inning (mph).

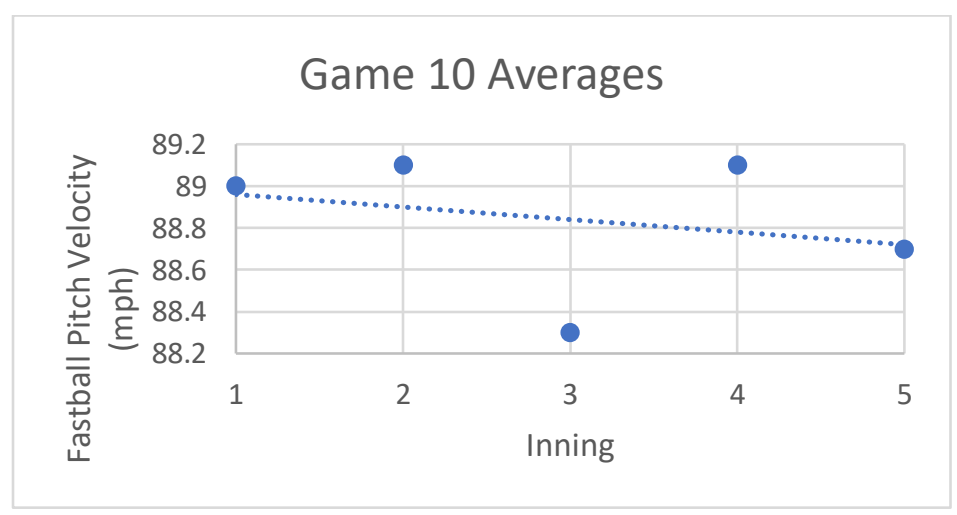


Figure D21. Game 11, Subject 1, Overall Fastball Pitch Velocity (mph).

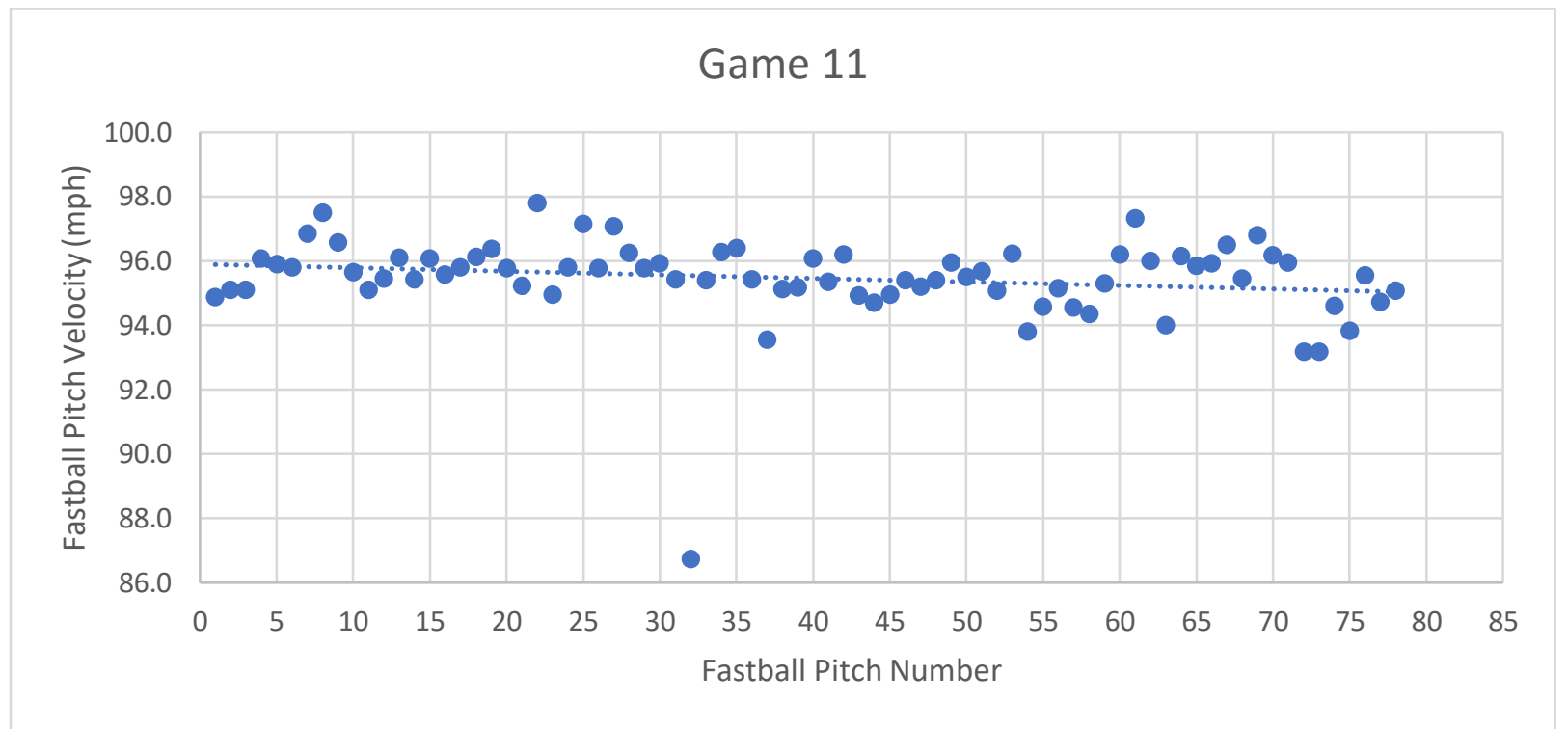

Figure D22. Game 11 Averages, Subject 1, Fastball Pitch Velocity Averages by Inning (mph).

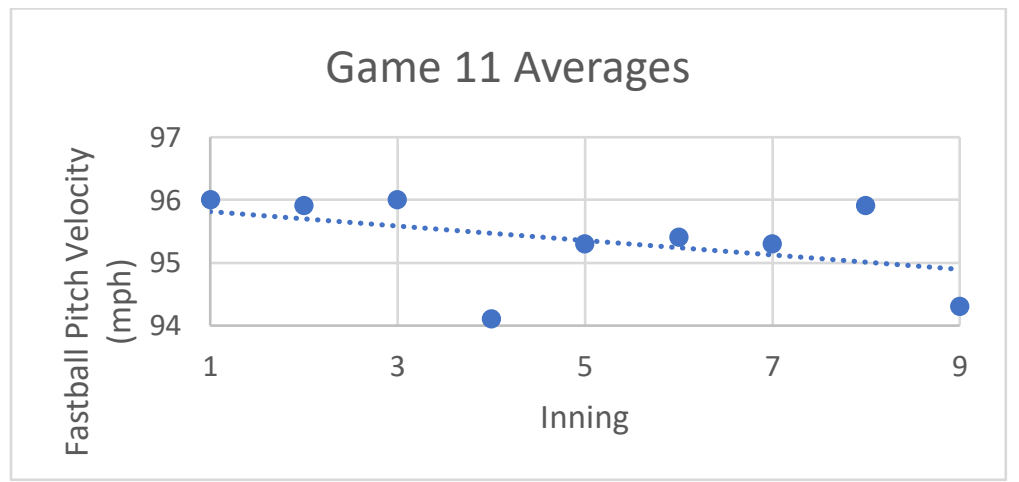


Figure D23. Game 12, Subject 2, Overall Fastball Pitch Velocity (mph).

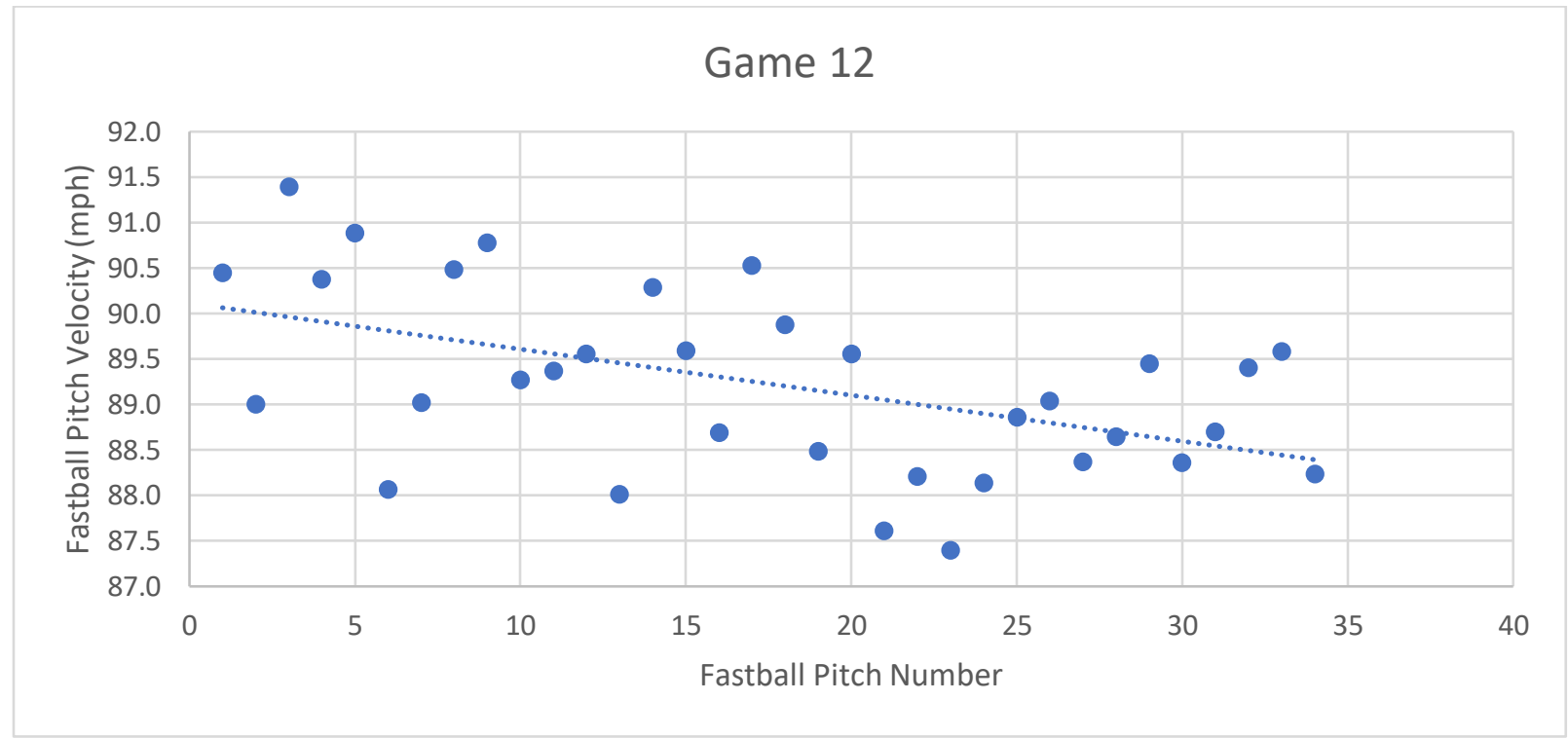

Figure D24. Game 12 Averages, Subject 2, Fastball Pitch Velocity Averages by Inning (mph).

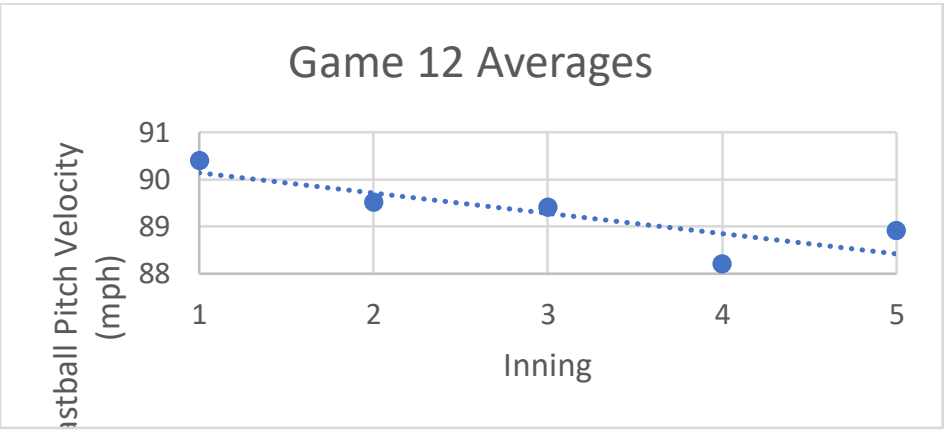

Figure D25. Game 13, Subject 3, Overall Fastball Pitch Velocity (mph).

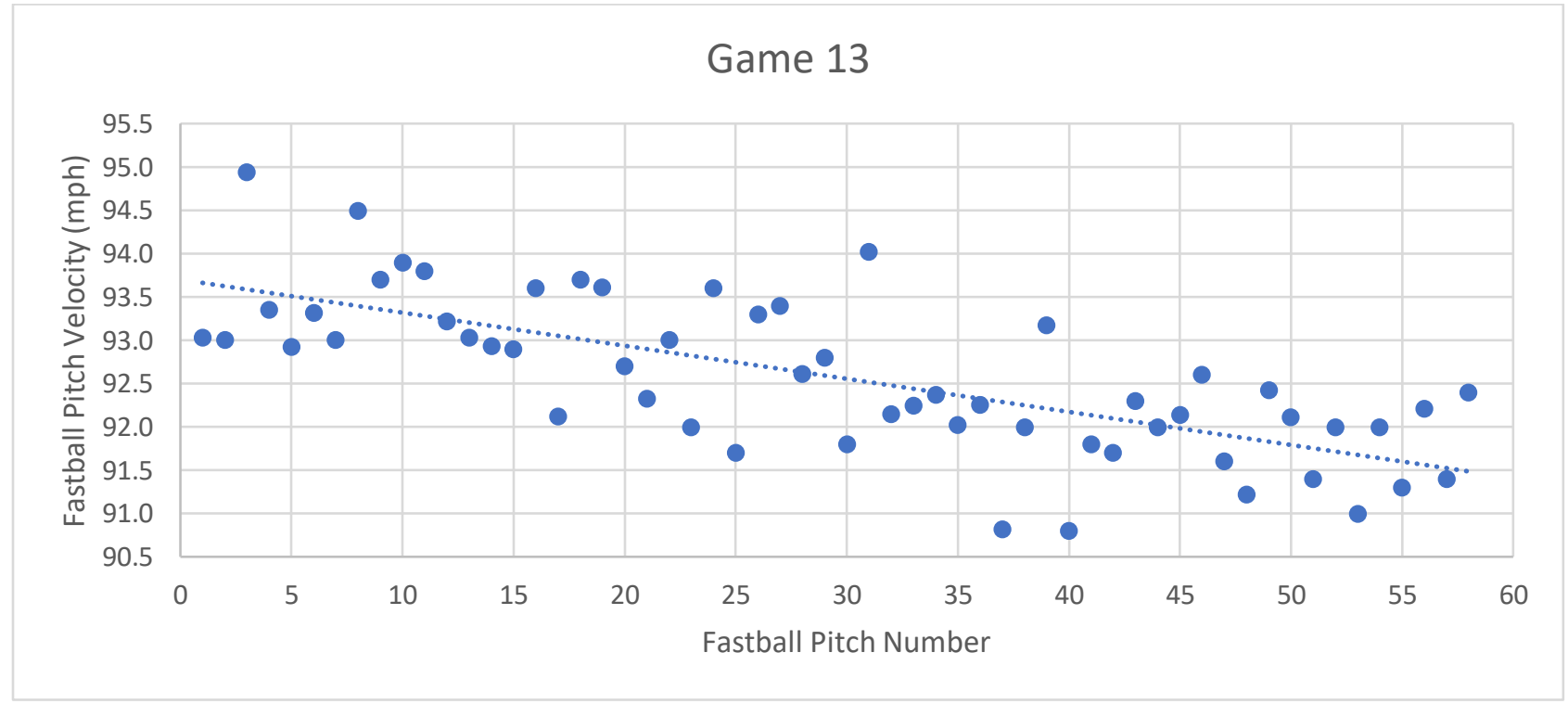


Figure D26. Game 13 Averages, Subject 3, Fastball Pitch Velocity Averages by Inning (mph).

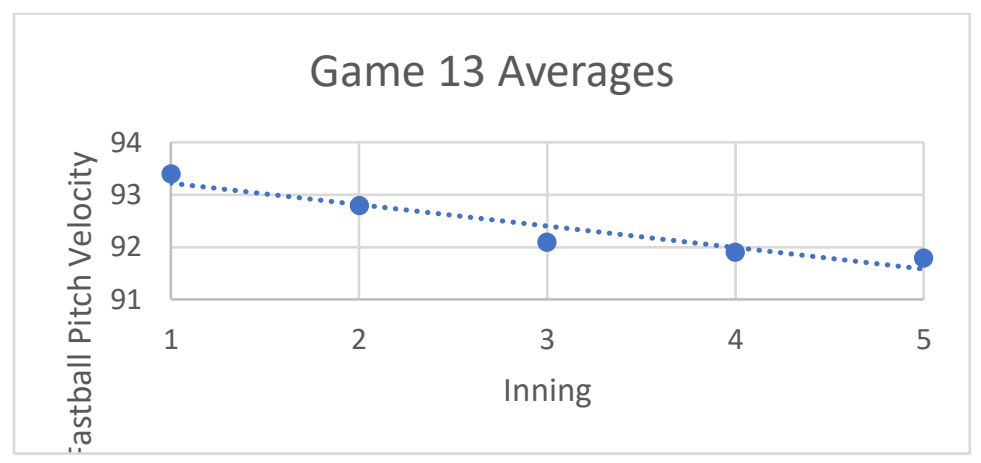

Figure D27. Game 14, Subject 1, Overall Fastball Pitch Velocity (mph).

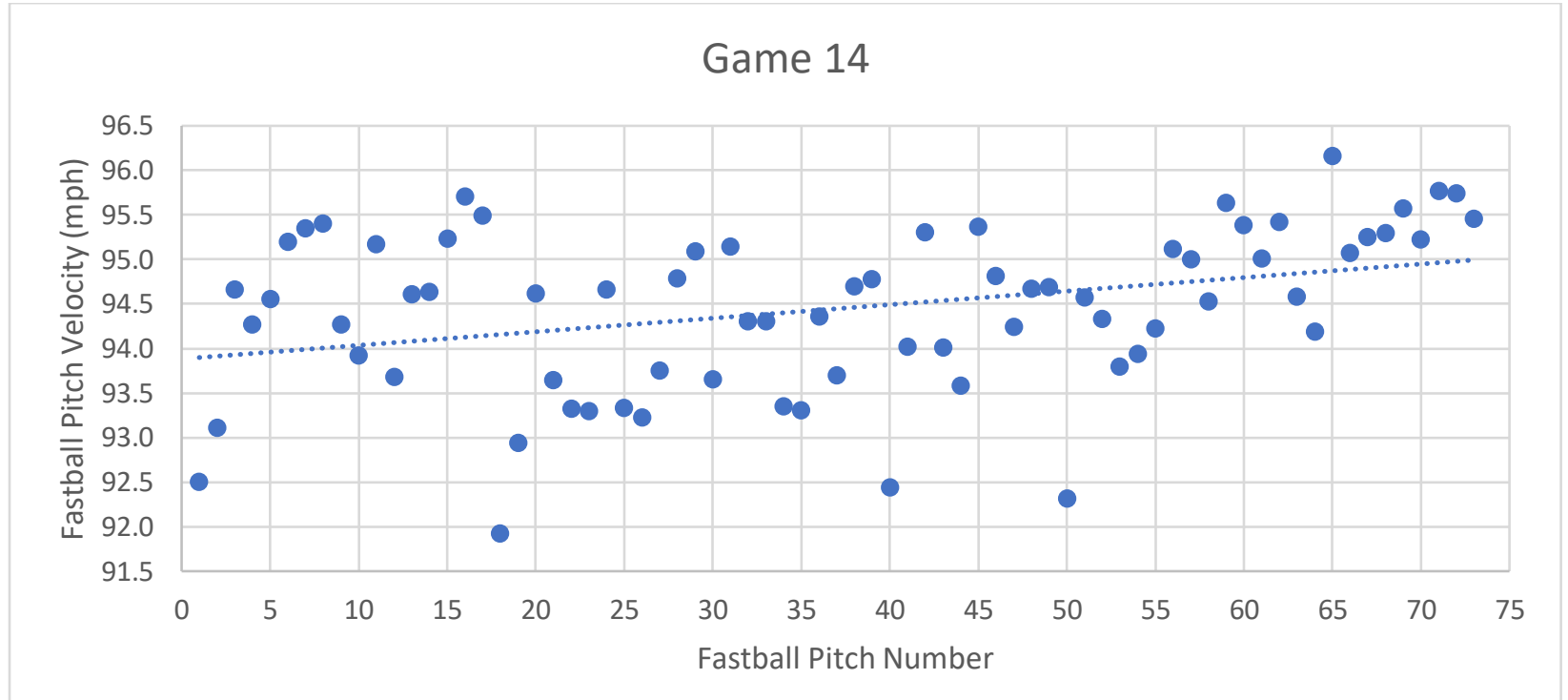

Figure D28. Game 14 Averages, Subject 1, Fastball Pitch Velocity Averages by Inning (mph).

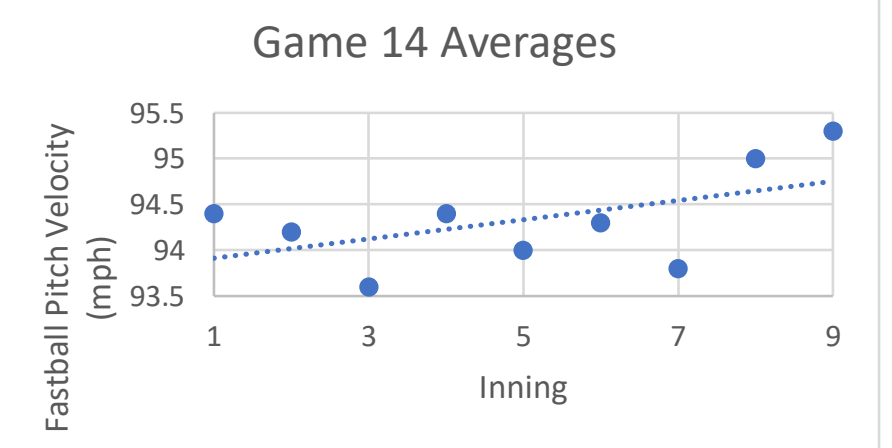


Figure D29. Game 15, Subject 2, Overall Fastball Pitch Velocity (mph).

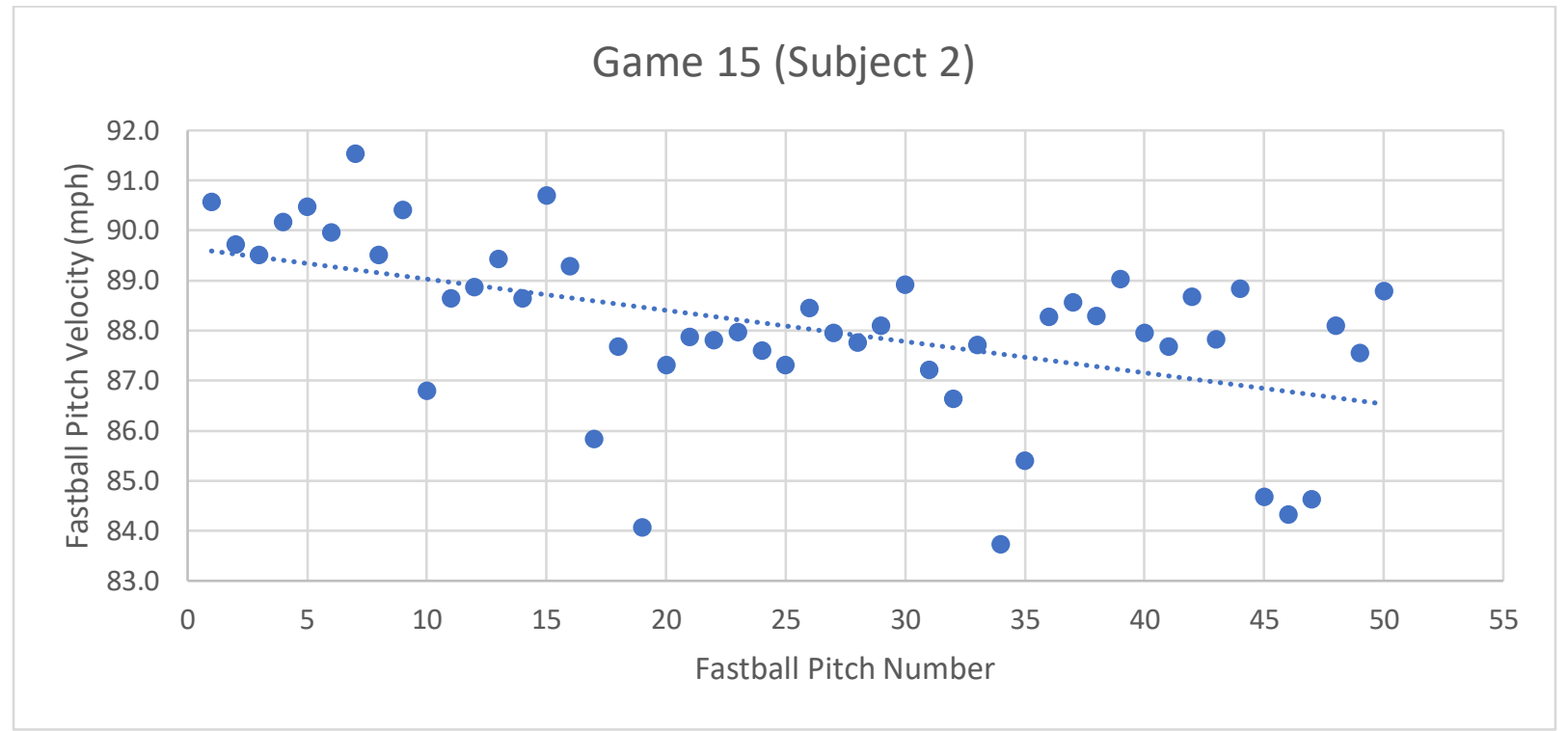

Figure D30. Game 15, Subject 4, Overall Fastball Pitch Velocity (mph).

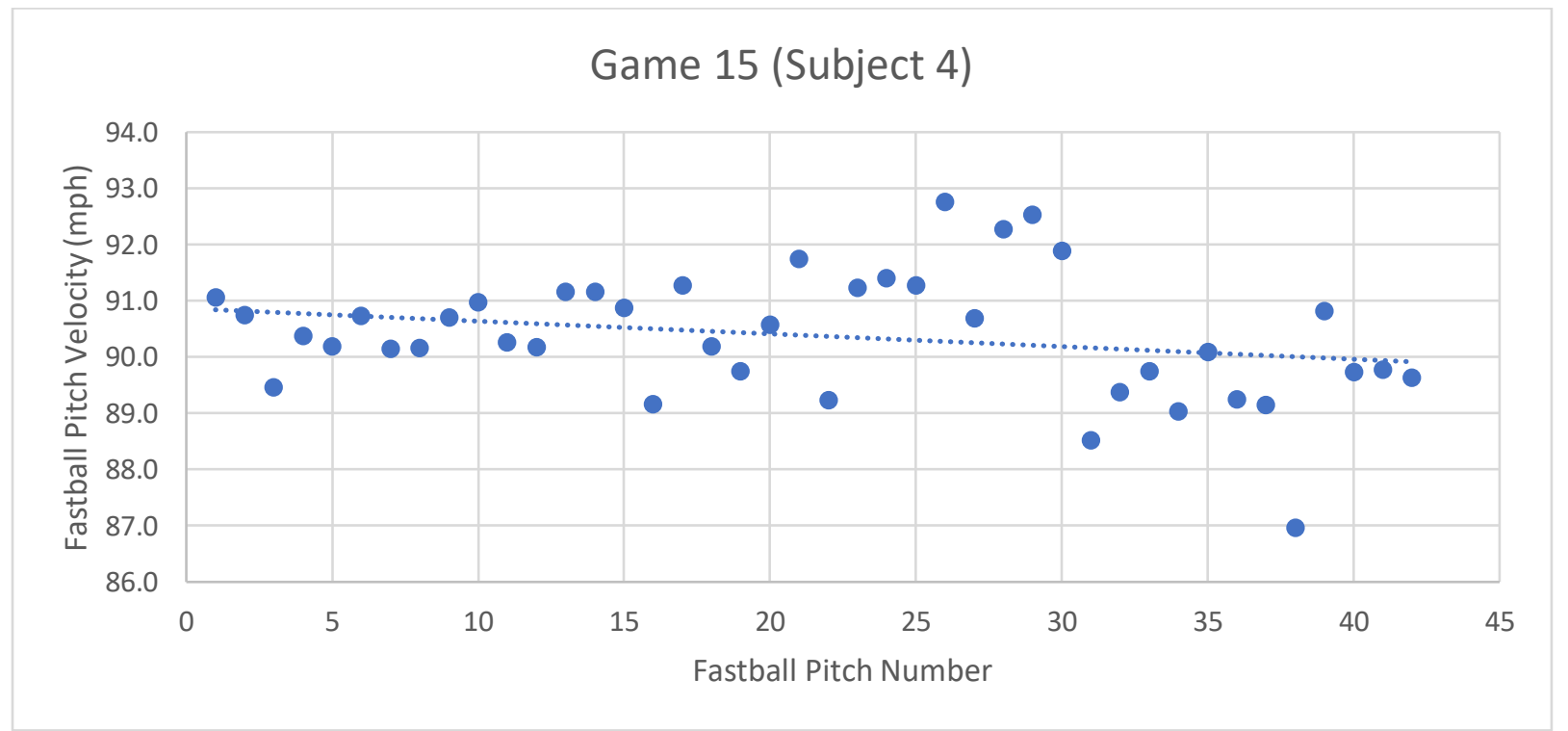


Figure D31. Game 15 Averages, Subject 2, Fastball Pitch Velocity Averages by Inning (mph).

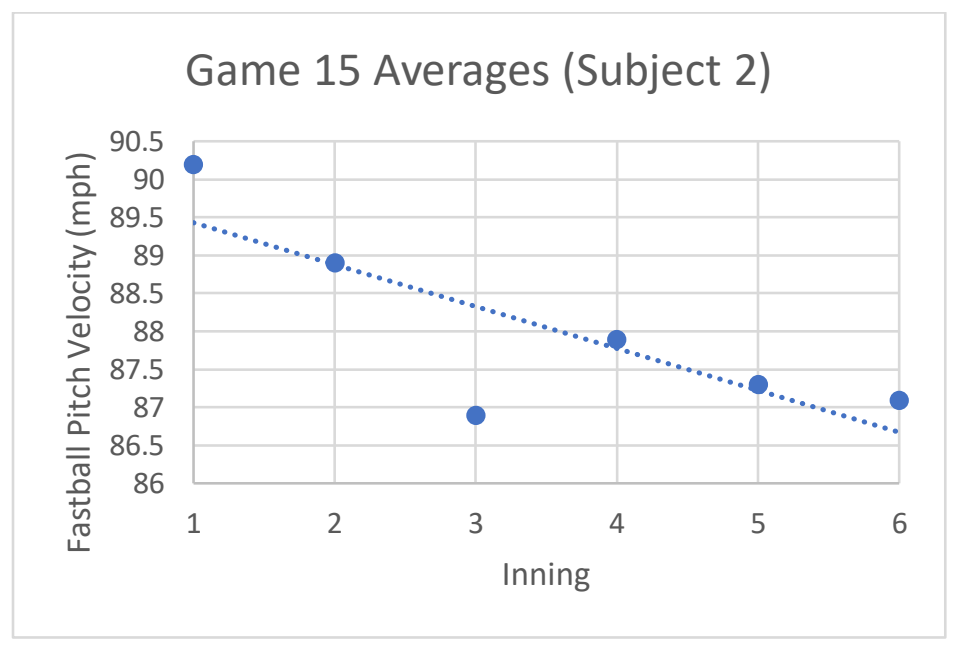

Figure D32. Game 15 Averages, Subject 4, Fastball Pitch Velocity Averages by Inning (mph).

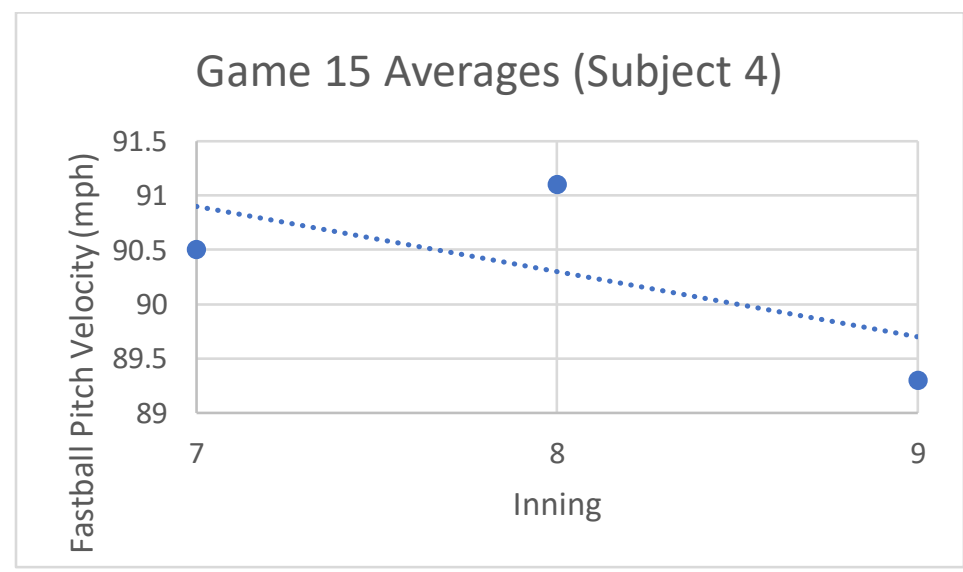

Figure D33. Game 16, Subject 3, Overall Fastball Pitch Velocity (mph).

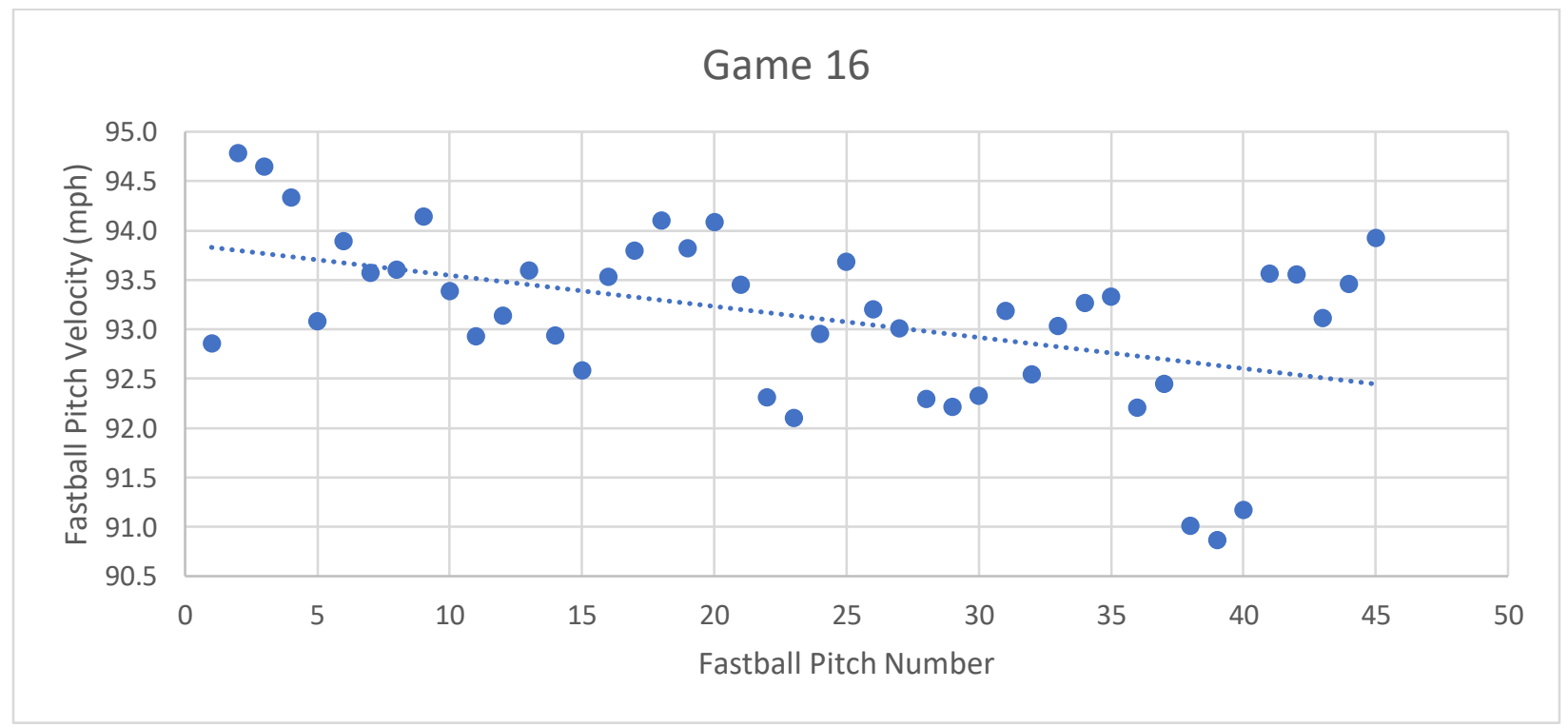


Figure D34. Game 16 Averages, Subject 3, Fastball Pitch Velocity Averages by Inning (mph).

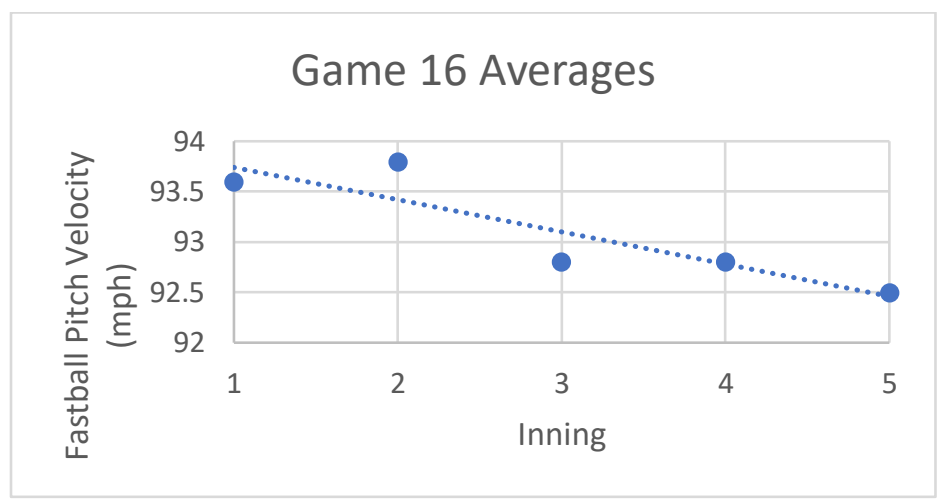

Figure D35. Game 17, Subject 3, Overall Fastball Pitch Velocity (mph).

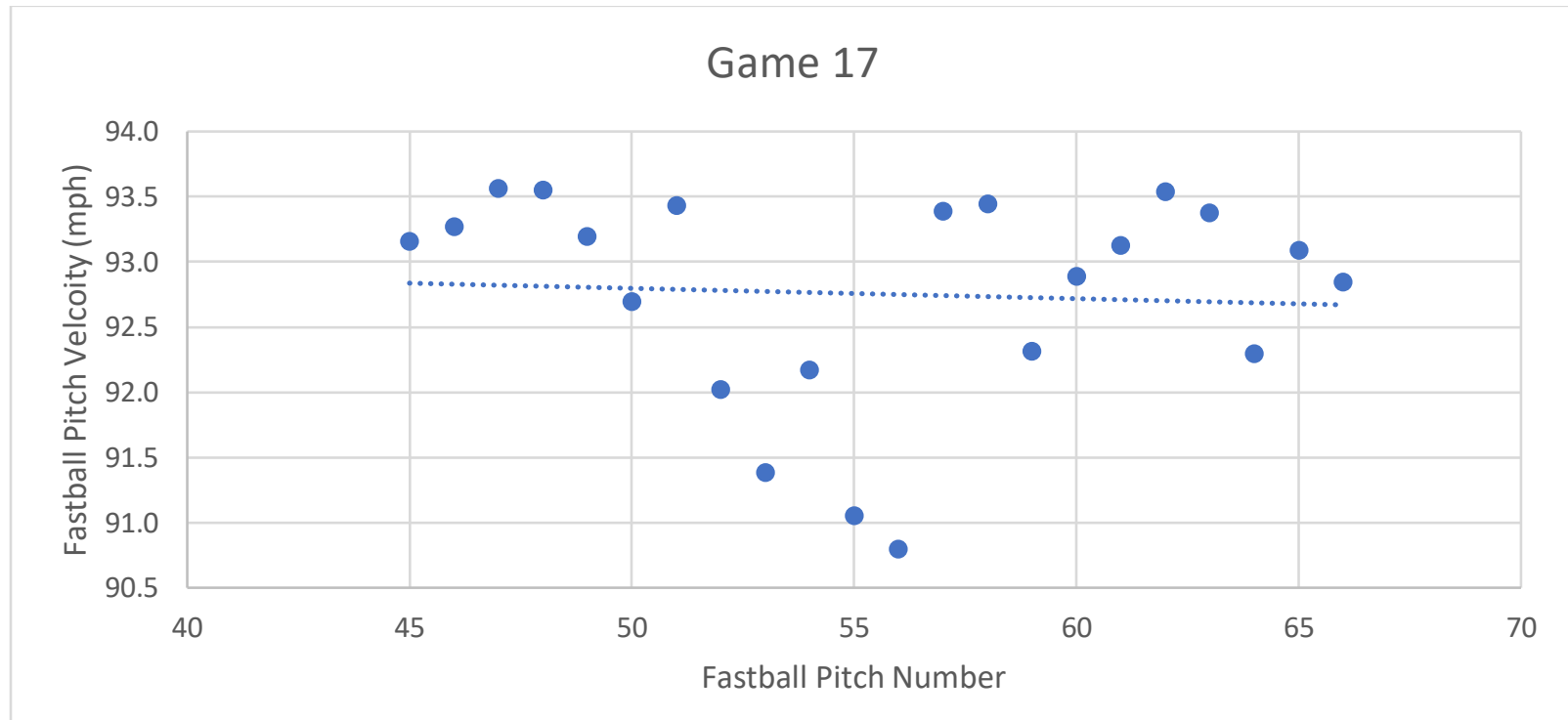

Figure D36. Game 17 Averages, Subject 3, Fastball Pitch Velocity Averages by Inning (mph).

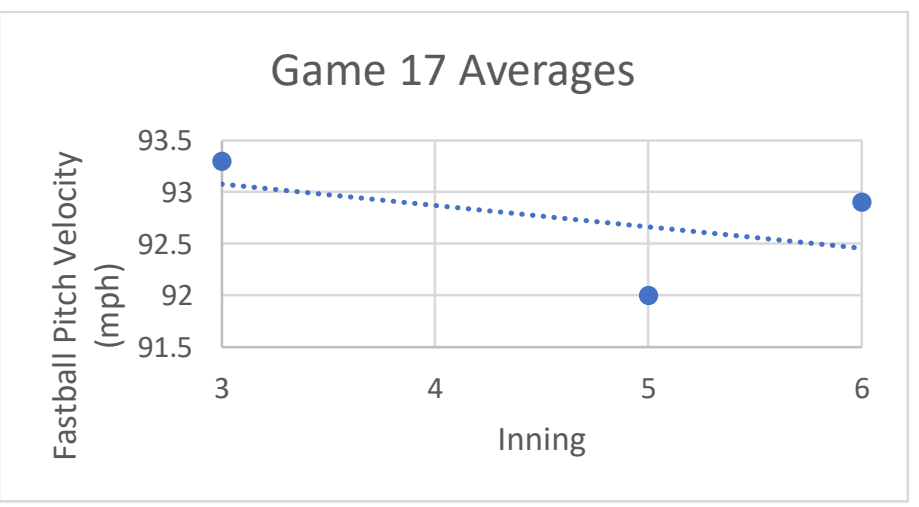


Figure D37. Subject 1 Fastball Pitch Velocity Averages by Game (mph).

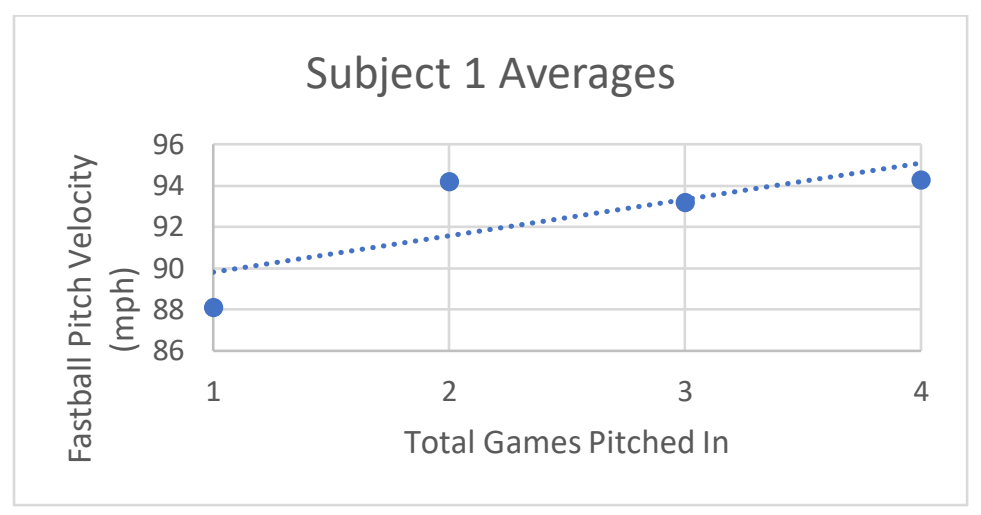

Figure D38. Subject 1 Fastball Pitch Velocity Averages by Inning (mph).

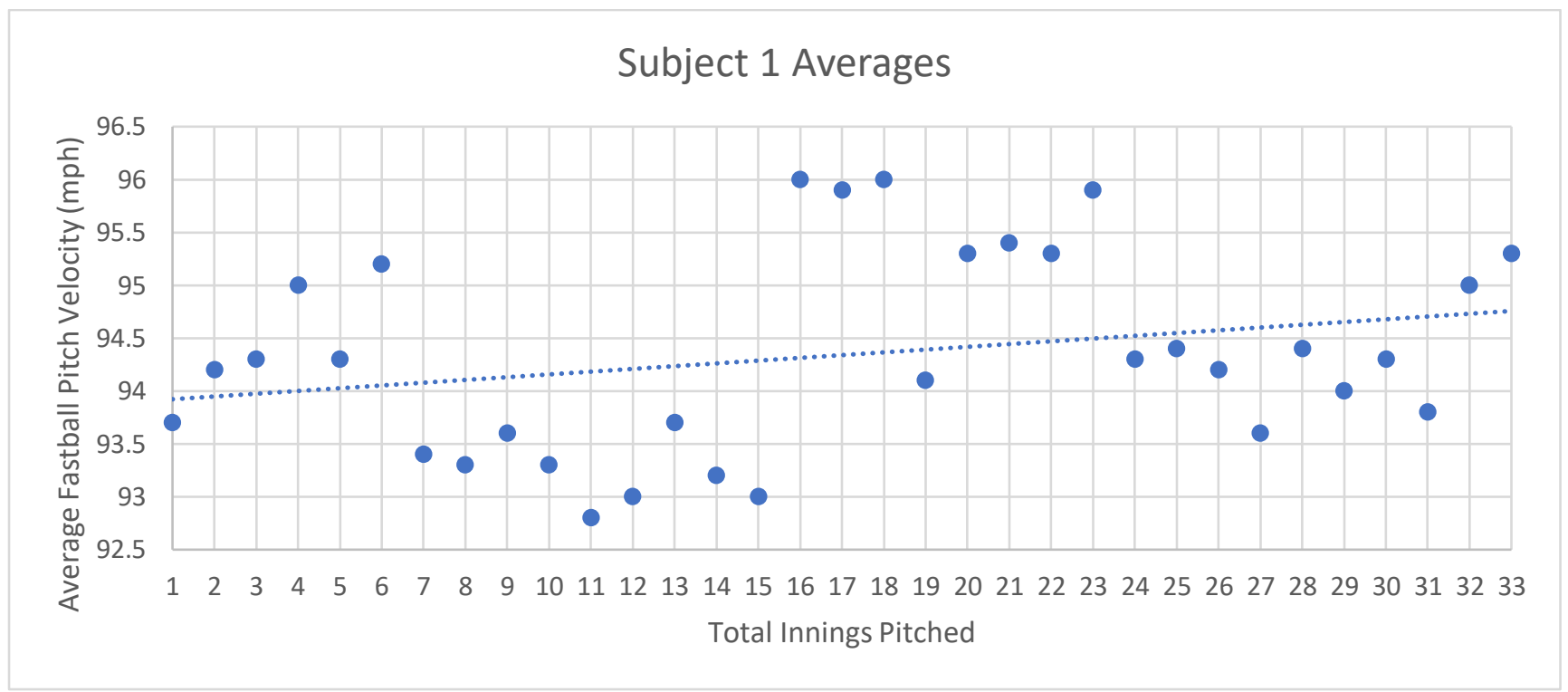

Figure D39. Subject 2 Fastball Pitch Velocity Averages by Game (mph).

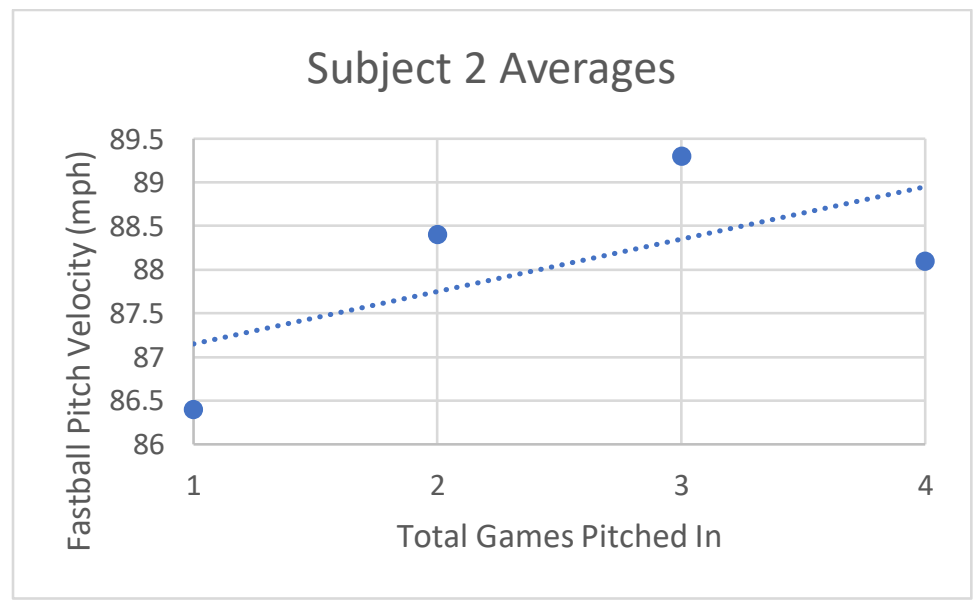


Figure D40. Fastball Pitch Velocity Averages by Inning (mph).

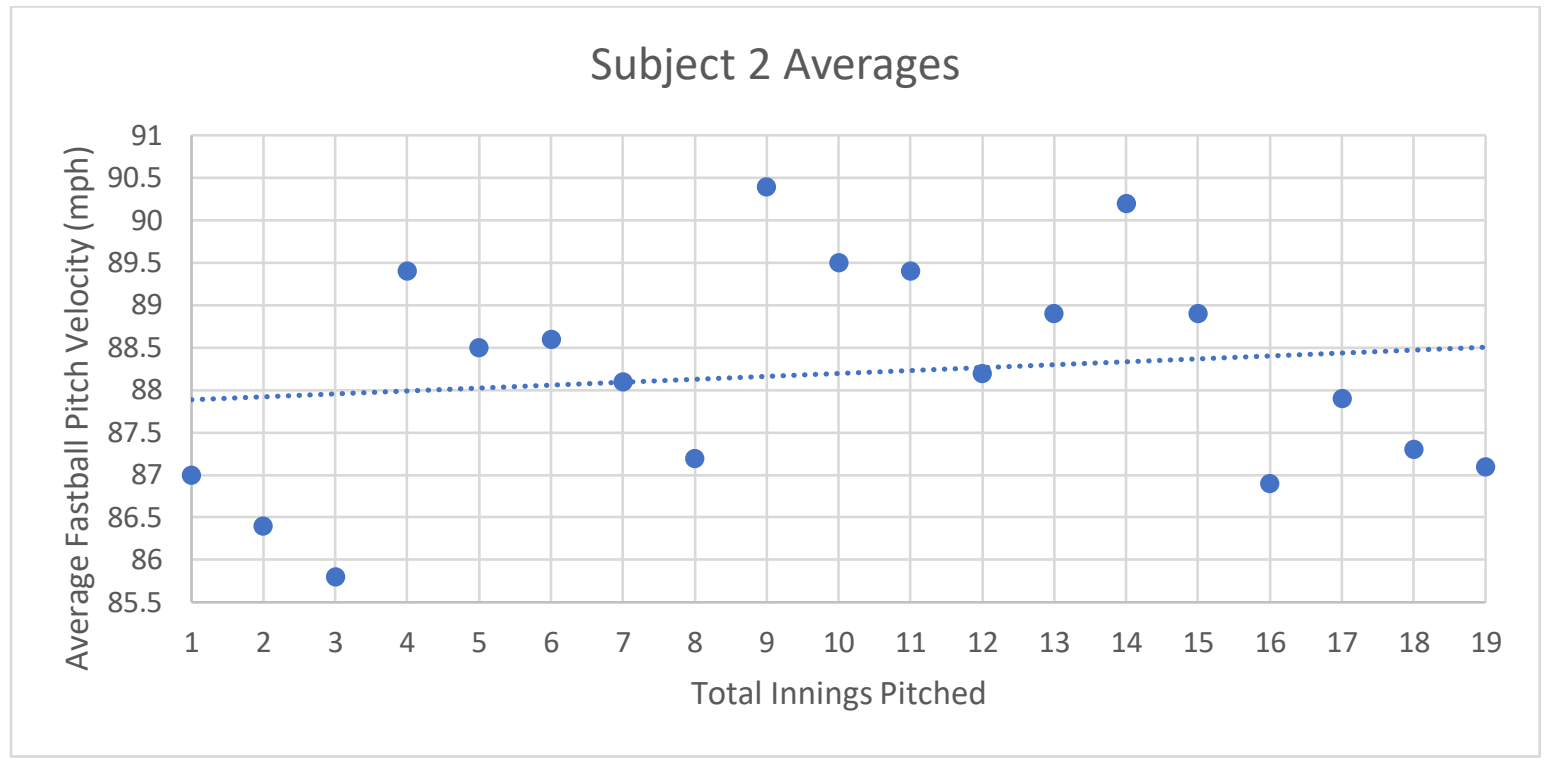

Figure D41. Subject 3 Fastball Pitch Velocity Averages by Game (mph).

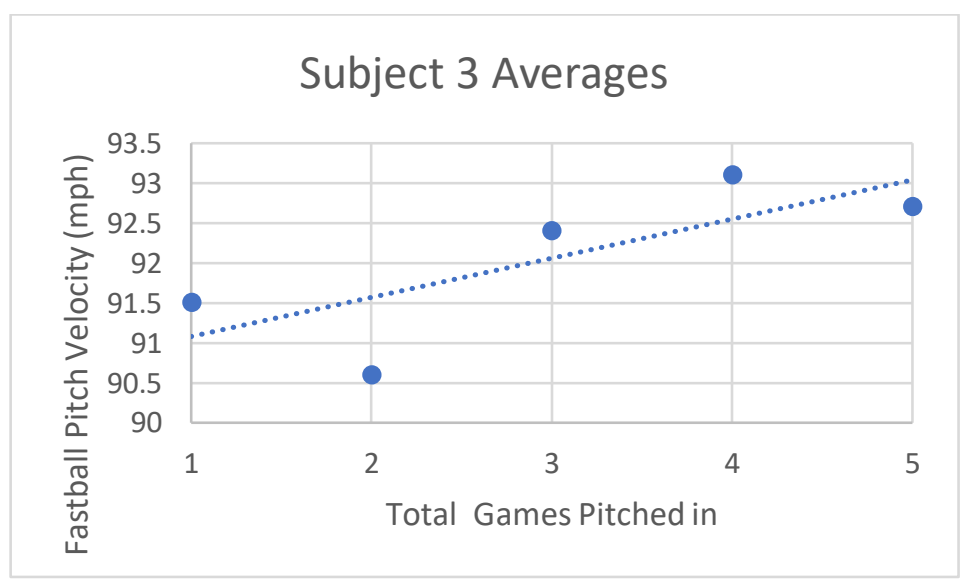


Figure D42. Subject 3 Fastball Pitch Velocity Averages by Inning (mph).

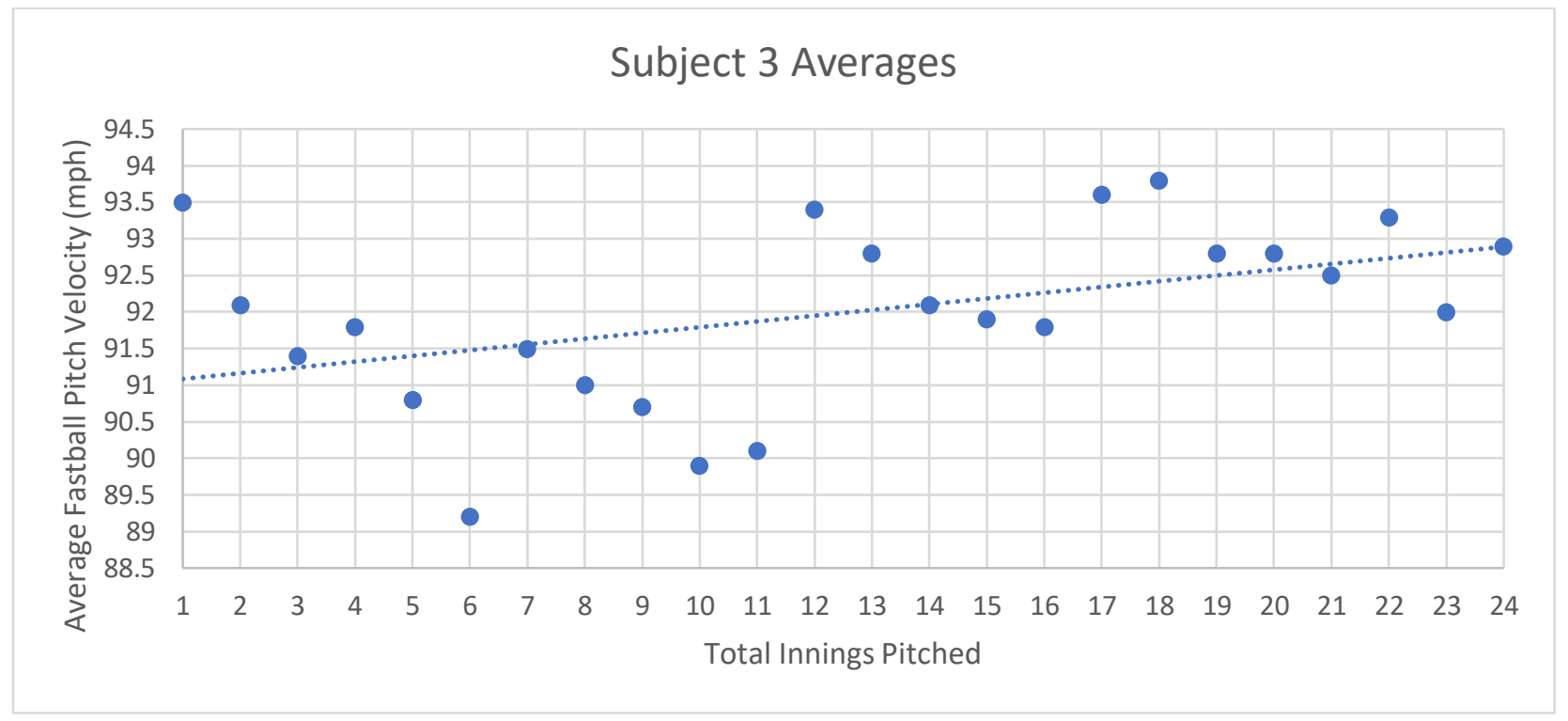

Figure D43. Subject 4 Fastball Pitch Velocity Averages by Game (mph).

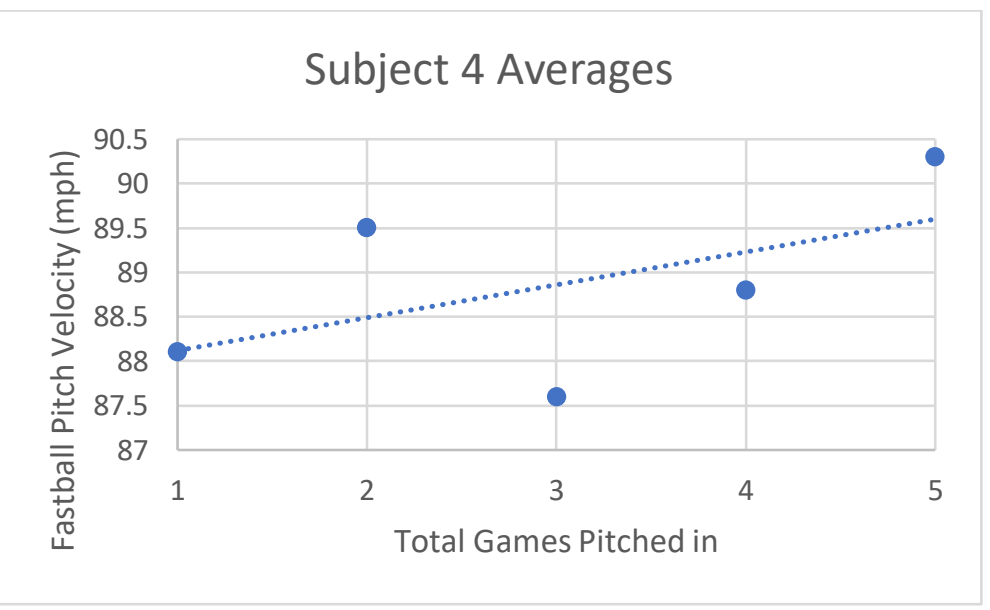


Figure D44. Subject 4 Averages by Inning.

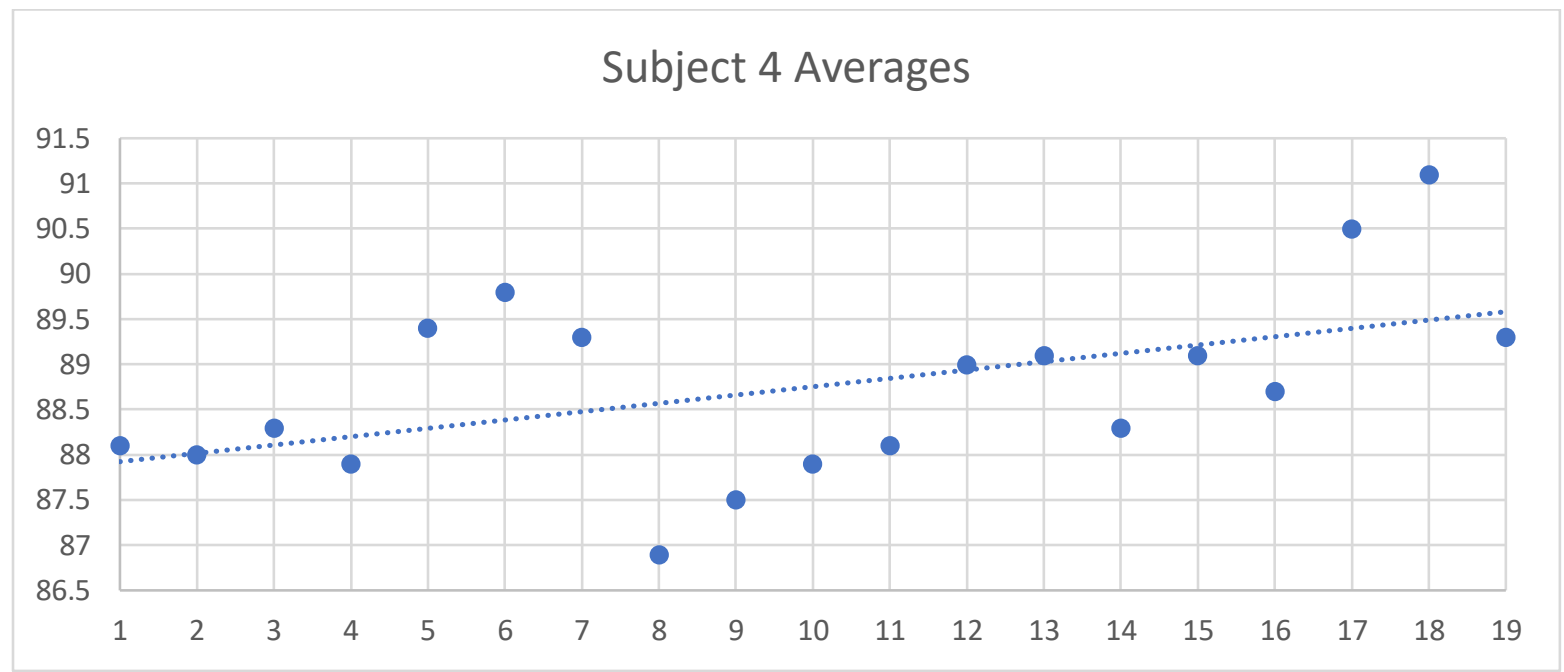




\section{APPENDIX E \\ RECOMMENDATIONS FOR FUTURE RESEARCH}

1. Repeat the study using a larger sample size by recruiting all pitchers (starting and bullpen) from the program as well as recruiting pitchers from other mid-Atlantic universities.

2. Repeat the study examining other pitching characteristics (release point, tilt, spin data, angle of release, hang time, etc.) throughout innings and games.

3. Repeat the study across multiple baseball seasons to observe longitudinal pitching changes.

4. Conduct an intervention study with a control group, comparable to the intervention group, that does not use BFR for recovery post-pitching bouts.

5. Repeat the study with more controlled variables in terms of other recovery methods implemented and strengthening and lifting sessions tracked.

6. Repeat the study in a simulated setting to allow for more control and utilize a biomechanical analysis to see if there were alterations in the throwing patterns for each subject throughout the season.

7. Repeat the study with a Delfi BFR unit.

8. Repeat the study using pitching data from both home and away games.

9. Repeat the study and incorporate subjective patient-oriented outcomes surveys such as rating of perceived exertion and compare with release point, tilt, spin data, angle of release, hang time, types of pitches etc. 


\section{ADDITIONAL REFERENCES}

36. Jessee MB, Mouser JG, Buckner SL, et al. Effects of load on the acute response of muscles proximal and distal to blood flow restriction. J Physiol Sci. 2018.

37. Tabata S, Suzuki Y, Azuma K, Matsumoto H. Rhabdomyolysis after performing blood flow restriction training: a case report. J Strength Cond Res. 2016:30(7):2064-2068.

38. Hughes L, Paton B, Rosenblatt B, Gissane C, Patterson SD. Blood flow restriction training in clinical musculoskeletal rehabilitation: a systematic review and meta-analysis. Br J Sports Med. 2017; 51:1003-1011.

39. Douris PC, Cogen ZS, Fields HT, et al. The effects of blood flow restriction training on functional improvements in an active single subject with parkinson disease. Int $J$ Sports Phys Ther. 2018;13(2):247-254.

40. Cook SB, JA Kanaley, LL Ploutz-Snyder. Neuromuscular function following muscular unloading and blood flow restricted exercise. Eur J Appl Physiol. 2014;114(7): 13571365.

41. Wilson JM, Lowery RP, Joy JM, et al. Practical blood flow restriction training increases acute determinants of hypertrophy without increasing indices of muscle damage. $J$ Strength Cond Res. 2013;27(11): 3068-3075.

42. Brandner CR, Kidgell DJ, Warmington SA. Unilateral bicep curl hemodynamics: lowpressure continuous vs high-pressure intermittent blood flow restriction. Scand J Med Sci Sports. 2015;25:770-777.

43. Stanford Health Care. Surgery Statistics. https://stanfordhealthcare.org/medicalclinics/surgery-clinic/patient-resources/surgery-statistics.html. Accessed 30 October 2018.

44. Ferraz RB, Gualano B, Rodreigues R, et al. Benefits of resistance training with blood flow restriction in knee osteoarthritis. ACSM. 2018;50(5):897-905.

45. Dankel SJ, Jessee MB, Abe T, Loenneke JP. The effects of blood flow restriction on upper-body musculature located distal and proximal to applied pressure. Sports Med. 2016;46(1):23-33.

46. Willis SJ, Alvarez L, Borrani F, Millet GP. Oxygenation time course and neuromuscular fatigue during repeated cycling sprints with bilateral blood flow restriction. Eur J Appl Physiol. 2018; 6(19):1-13.

47. Loennecke JP, Fahs CA, Wilson JM, Bemben MG. Blood flow restriction: the metabolite/volume threshold theory. 2011;77(5):748-752. 
48. Conceicão MS, Gáspari1 AF, Ramfrapes APB, et al. Anaerobic metabolism induces greater total energy expenditure during exercise with blood flow restriction.

2018;13(3):1-13.

49. Yamanaka T, Farley RS, Caputo JL: Occlusion training increases muscular strength in division IA football players. J Strength Cond Res. 2012;26:2523-2529.

50. Tanaka Y, Takarada Y. The impact of aerobic exercise training with vascular occlusion in patients with chronic heart failure. Eur Heart J. 2018; 5(4):586-591..

51. Taylor JL, Amann M, Duchateau J, Meeusen R, Rice CL. Neural contributions to muscle fatigue: from the brain to the muscle and back again. Med Sci Sports Exerc. 2016;48(11):2294-2306.

52. Lyman S, Fleisig GS, Andrews JR, Osinski ED. Effect of pitch type, pitch count, and pitching mechanics on risk of elbow and shoulder pain in youth baseball pitchers. $A m J$ Sports Med. 2002;30(4):463-468.

53. Makhni EC, Morrow ZS, Luchetti TJ et al. Arm pain in youth baseball players: a survey of healthy players. Am J Sports Med. 2015:43(1);41-6.

54. Bishop SH, Herron RL, Ryan GA, Katica CP, Bishop PA. The effect of intermittent arm and shoulder cooling on baseball pitching velocity. J Strength Cond Res.

2016;30(4):1027-1032. 